\title{
Modeling and Control of Non-laminated Active Magnetic Thrust Bearings
}

A Thesis
Presented to
the Faculty of the School of Engineering and Applied Science
University of Virginia
In Partial Fulfillment
of the requirements for the Degree
Master of Science (Mechanical and Aerospace Engineering)
by
Deckary W. Whitlow 2014




\section{Abstract}

Non-laminated magnetic thrust bearings exhibit reduced dynamic performance, compared to laminated radial bearings, due to eddy current effects. Segmented thrust bearing stators have been introduced to increase actuator performance by disrupting eddy current paths in the same manner as laminations in radial magnetic bearings. However, due to manufacturing limitations, thrust bearing stators cannot be easily segmented to the extent that they would be considered fully laminated. Therefore, eddy currents continue to affect their dynamic performance significantly. This work aims to improve the performance of non-laminated thrust active magnetic bearings through improved modeling and control design.

Currently, accurate modeling of segmented stator performance relies on finite element analysis, which is a time consuming process. In this work, an analytic model of cylindrical segmented electromagnetic actuators, including eddy currents effects, is developed. The model is an extension of the analytic model for C-type

electromagnetic actuators developed by Zhu et al., [1, 2]. Zhu's work on cylindrical magnetic actuators, $[3,2]$ is also continued in order to develop an analytic model for cylindrical electromagnetic actuators with a center hole, i.e. non-laminated active magnetic thrust bearings. All analytic models developed in this work are verified via finite element analysis.

Based on analytic and finite element modeling, it is found that thrust bearing stator segmentation results in dramatic improvements in dynamic performance. With six stator cuts, and depending on the specific geometry, bandwidth from current input to force output is typically improved by more than 2-fold.

The potential for dynamic performance improvements for non-laminated magnetic thrust bearings by non-linear and dynamic compensation is also investigated. Simulations using a detailed non-linear model suggest that compensation, combined with proportional-integral-derivative control, will improve performance consistency and disturbance rejection for non-laminated thrust bearings compared to performance with PID alone. 


\section{Approval Sheet}

This thesis is submitted in partial fulfillment of the requirements for the degree of Master of Science (Mechanical and Aerospace Engineering)

Zackary W. Whitlow

This thesis has been read and approved by the Examining Committee:

Roger L. Fittro, Adviser

Carl R. Knospe, Adviser

Gavin Garner, Committee Chair

George Gillies

Accepted for the School of Engineering and Applied Science:

James H. Aylor, Dean, School of Engineering and Applied Science

December 2014 
To my family 


\section{Acknowledgments}

I would like to thank all of the ROMAC students and staff for welcoming me into the lab and being so willing to help whenever I had questions. Thanks to Lori for taking care of whatever problems I encountered and for providing coffee while I was struggling to finish my work. Thanks to Ali for his help with any question related to math and for many helpful discussions. Special thanks to X and Dee for many discussions about magnetic bearings, control, and food. It has been great sharing an office space with you and I will miss you both.

I thank Professors Fittro and Knospe for their guidance and discussions on magnetic bearings and control. I have learned so much talking with you and seeing the way you approach problems. It has been a great opportunity to work with you both.

Thanks to Professor Garner for his willingness to help and for sharing his knowledge of mechatronics. What I learned has inspired me to focus on mechatronics and controls and has opened up many opportunities already.

I thank my wife Kyle for encouraging me to pursue an engineering degree and for supporting me throughout the process. I would not have done this without her encouragement and support and I'm grateful. I also must thank my daughter for providing some perspective and for always welcoming me home. 


\section{Contents}

Contents $\quad \mathbf{f}$

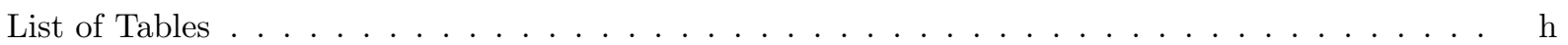

List of Figures . . . . . . . . . . . . . . . . . . . . . . . . . . i

1 Introduction 1

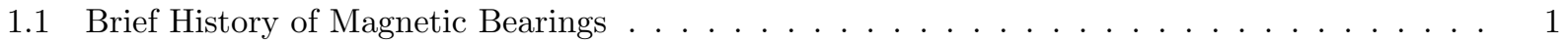

1.2 Introduction to Active Magnetic Bearings . . . . . . . . . . . . . . . . . . . . . . 3

1.3 Analytic Modeling of Non-laminated Magnetic Actuators . . . . . . . . . . . . . . 6

1.4 Fractional-Order Differential Equations . . . . . . . . . . . . . . . . . . . . . 7

1.5 Segmentation of Thrust AMBs to Enhance Dynamic Performance . . . . . . . . . . . . . . . . 9

1.6 Feedback Linearization . . . . . . . . . . . . . . . . . . . . . . . . . . 10

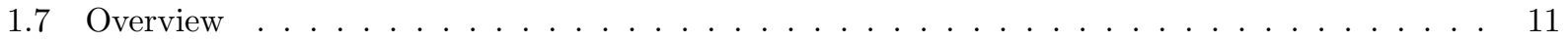

2 Analytic Model for Non-laminated Thrust AMBs 13

2.1 Current Model for Axisymmetric Actuators . . . . . . . . . . . . . . . . . . . . 13

2.2 Model for Axisymmetric Actuators With a Center Hole . . . . . . . . . . . . . . . . . 16

2.3 Approximate Analytic Model . . . . . . . . . . . . . . . . . . . . . . . . 20

2.4 Current to Force Transfer Function . . . . . . . . . . . . . . . . . . . . . . . . . . . . . . . 22

2.5 Verification of the Analytic Model . . . . . . . . . . . . . . . . . . . . . 24

3 Analytic Model for Segmented Thrust AMBs 33

3.1 Analysis of Extending Flotor . . . . . . . . . . . . . . . . . . . . . . . 34

3.2 Zhu's Model for C-type Actuators . . . . . . . . . . . . . . . . . . . . . . . . . . . 34

3.3 Analytic Model for Segmented Thrust AMBs . . . . . . . . . . . . . . . . . . . . . 38

3.4 3-D Finite Element Analysis . . . . . . . . . . . . . . . . . . . . . . . . . . . . . 40

3.5 Verification of the Analytic Model . . . . . . . . . . . . . . . . . . . . . . 41

3.6 Dynamic Performance Enhancement by Stator Segmentation . . . . . . . . . . . . . . . . . 43

3.7 Flotor Segmentation . . . . . . . . . . . . . . . . . . . . . . 43

4 Control of Non-laminated AMBs Using Feedback Linearization 58

4.1 Feedback Linearization for Magnetic Bearings . . . . . . . . . . . . . . . . . . . . . . . 59

4.2 Nonlinear Modeling . . . . . . . . . . . . . . . . . . . . . . . . . . . 60

4.3 Feedback Linearization . . . . . . . . . . . . . . . . . . . . . . . . . 65

4.4 Control Design . . . . . . . . . . . . . . . . . . . . . . . . . 66

4.5 Linear Performance Analysis . . . . . . . . . . . . . . . . . . . . . . . . . 68

4.6 Nonlinear Performance Analysis . . . . . . . . . . . . . . . . . . . . . . . . . 71

4.7 Analysis of Robustness . . . . . . . . . . . . . . . . . . . . . . . . . . . 73

4.8 Summary ... . . . . . . . . . . . . . . . . . . . . 74 
5 Conclusions and Future Work $\quad 89$

5.1 Analytic Models ... . . . . . . . . . . . . . . . . . . . . . . . 90

5.2 Performance of Segmented Thrust AMBs . . . . . . . . . . . . . . . . . . . . . . . 90

5.3 Feedback Linearization and Control . . . . . . . . . . . . . . . . . . . . . . . 91

5.4 Future Work . . . . . . . . . . . . . . . . . . . . . . . . 92

Appendices $\mathbf{9 3}$

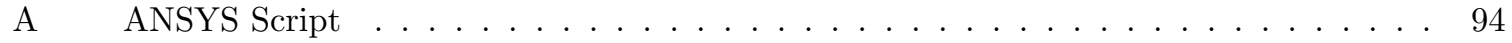

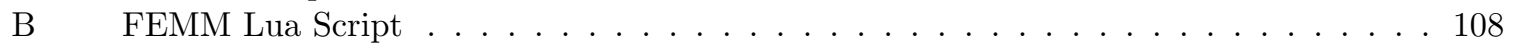

Bibliography 


\section{List of Tables}

2.1 Effective reluctances for Regions 1 through $6 \ldots \ldots \ldots \ldots \ldots \ldots \ldots$

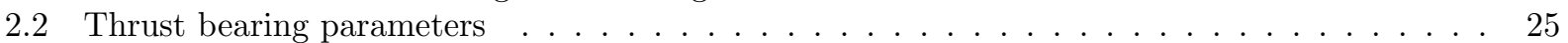

3.1 Guidlines for adaptation of segmented thrust AMB geometry to C-type analytic model. . . . 42

4.1 AMB Test Case Parameters . . . . . . . . . . . . . . . . . . . . . . . . . . . . 67

4.2 Closed Loop Performance . . . . . . . . . . . . . . . . . . . . . . . 68

4.3 Linear Robustness Parameters _ . . . . . . . . . . . . . . . . . . . . . . 71 


\section{List of Figures}

1.1 Drawing of Jesse Beams vacuum-type magnetically suspended ultracentrifuge. Shaft (A), rotor $(C)$, induction motor $(F)$, suspension electromagnet $(M)$, magnetic suspension core $(R)$, coil windings $(L)$, oil gland $\left(G_{1}\right)$, oil bearing $\left(G_{2}\right.$ and $\left.G_{3}\right)$, viscous damper $\left(G_{4}\right)$, vacuum chamber $(\mathrm{V})$. Figure adapted from $[4] . \ldots \ldots \ldots \ldots \ldots \ldots$

1.2 Cross section of an eight stage compressor supported by magnetic bearings. Figure adapted

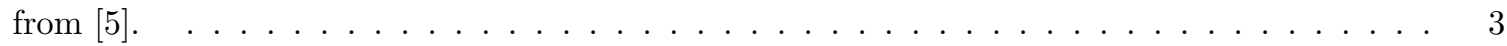

1.3 Thrust active magnetic bearing. Cross section (left) and axial view. Figure adapted from [6].

1.4 Thrust AMB system showing thrust disk portion of a rotor and the loop between rotor position and AMB including, axial position sensor, sensor signal conditioning, digital controller, power

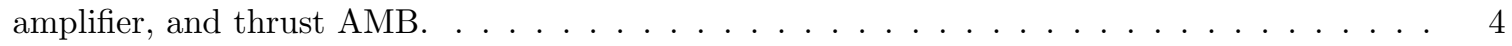

1.5 Depiction of a traditional thrust stator and a segmented stator (right) . . . . . . . . . 5

1.6 Depiction of a single axis of a laminated radial active magnetic bearing. . . . . . . . . . 6

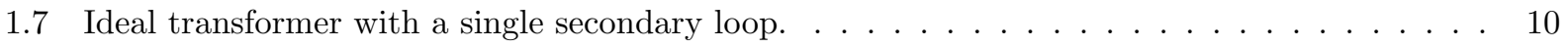

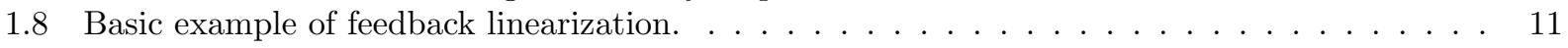

2.1 Parameters describing axisymmetric actuator geometry without a center hole.Adapted from [3]. 14

2.2 Air gap flux density varies with radial position. . . . . . . . . . . . . . . . . 14

2.3 Flux contours of geometry with a center hole with a static current excitation. . . . . . . . 16

2.4 Flux contours real (left) and imaginary (right) from FEA with a $50 \mathrm{~Hz}$ excitation current. . . 16

2.5 Axisymmetric geometry divided into six regions, each with an effective reluctance dependent

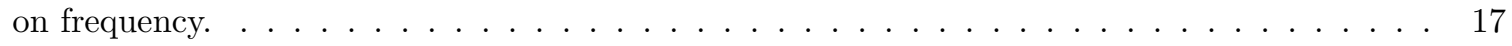

2.6 Zhu's reluctance network for Region $1 . \ldots \ldots \ldots \ldots \ldots \ldots \ldots$

2.7 Magnitude and phase of reluctance for Regions 1 and 6 with $r_{0}>0$ approximated using Taylor series or ad hoc method. . . . . . . . . . . . . . . . . . . . . . 22

2.8 2-D finite element modeling shows the flux magnitude and phase for three mesh resolutions. . 25

2.9 Case 1 (compressor thrust AMB): Flux magnitude and phase predicted by FEA model without flux leakage or fringing (FEA), with leakage and fringing (FEA LF), the full analytic model (Full), or the simplified analytic model (Approx.). Additive, Multiplicative, and Phase Error are also shown for the full and approximate analytic models relative to the FEA models with and without leakage and fringing. . . . . . . . . . . . .

2.10 Case 2 (1mm air gap): Flux magnitude and phase predicted by FEA model without flux leakage or fringing (FEA), with leakage and fringing (FEA LF), the full analytic model (Full), or the simplified analytic model (Approx.). Additive, Multiplicative, and Phase Error are also shown for the full and approximate analytic models relative to the FEA models with and

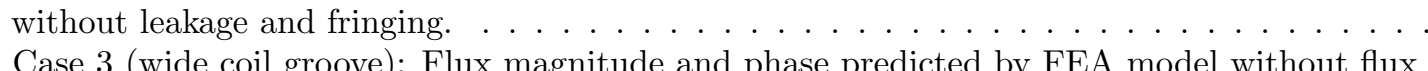

2.11 Case 3 (wide coil groove): Flux magnitude and phase predicted by FEA model without flux leakage or fringing (FEA), with leakage and fringing (FEA LF), the full analytic model (Full), or the simplified analytic model (Approx.). Additive, Multiplicative, and Phase Error are also shown for the full and approximate analytic models relative to the FEA models with and without leakage and fringing. . . . . . . . . . . . . . . 
2.12 Case 4 (deep coil groove): Flux magnitude and phase predicted by FEA model without flux leakage or fringing (FEA), with leakage and fringing (FEA LF), the full analytic model (Full), or the simplified analytic model (Approx.). Additive, Multiplicative, and Phase Error are also shown for the full and approximate analytic models relative to the FEA models with and without leakage and fringing.

2.13 Case 5 (large inner radius): Flux magnitude and phase predicted by FEA model without flux leakage or fringing (FEA), with leakage and fringing (FEA LF), the full analytic model (Full), or the simplified analytic model (Approx.). Additive, Multiplicative, and Phase Error are also shown for the full and approximate analytic models relative to the FEA models with and without leakage and fringing. . . . . . . . . . . . . . . . .

2.14 Case 6 (small inner radius): Flux magnitude and phase predicted by FEA model without flux leakage or fringing (FEA), with leakage and fringing (FEA LF), the full analytic model (Full), or the simplified analytic model (Approx.). Additive, Multiplicative, and Phase Error are also shown for the full and approximate analytic models relative to the FEA models with and without leakage and fringing. . . . . . . . . . . . . . . . .

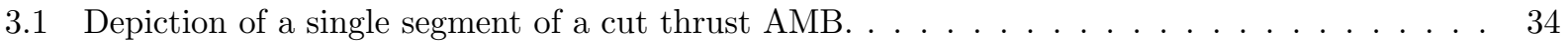

3.2 Flux contours with oversized flotor, static perturbation current. . . . . . . . . . . . 35

3.3 Flux contours real (left) and imaginary (right) with oversized flotor, $50 \mathrm{~Hz}$ perturbation current. 35

3.4 Total flux, magnitude and phase, for standard (Chapter 2 Case 1, and overhung flotor analyzed

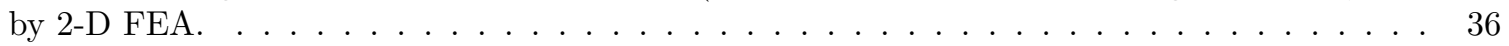

3.5 C-type actuator geometry with parameters. . . . . . . . . . . . . . 36

3.6 3-D FEA results showing normallized flux magnitude and phase for an uncut stator with a flotor with 0 (0s0f) or 1 cut (0s1f) and a stator with a single cut with flotor with 0 (1s0f) or 1

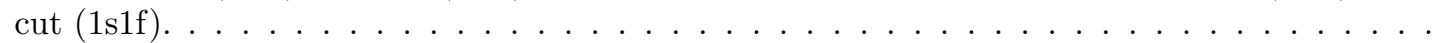

3.7 Finite element modeling with perturbation current input and magnetic flux output shows magnitude and phase for a standard and fine mesh resolutions. 2-D FEA results, FEMM, are included as a reference. . . . . . . . . . . . . . . . . . .

3.8 Magnetic flux density magnitude for a stator, case 1 , with 0 or 16 cuts. The flotor is not cut.

3.9 FEA Magnitude and phase from of thrust bearing, case 1, with unsegmented stator or cut stator. 48

3.10 Case 1 (compressor thrust AMB) - frequency response for 1 to 16 stator cuts. . . . . . . . . 49

3.11 Case $2(1 \mathrm{~mm}$ air gap) - frequency response for 1 to 16 stator cuts. . . . . . . . . . . . 50

3.12 Case 3 (wide coil groove) - frequency response for 1 to 16 stator cuts. . . . . . . . . . . . 51

3.13 Case 4 (deep coil groove) - frequency response for 1 to 16 stator cuts. . . . . . . . . . . . 52

3.14 Case 5 (large inner radius) - frequency response for 1 to 16 stator cuts. . . . . . . . . . . . 53

3.15 Case 6 (small inner radius) - frequency response for 1 to 16 stator cuts. . . . . . . . . . . 54

3.16 Bandwidth as a function of stator cuts, for each test case. . . . . . . . . . . . . . 55

3.17 Analytic analysis, using the full model, of the effect of a single flotor cut on dynamic performance of Case 1 with 2, 4, 8, and 16 cuts. Labels nsmf correspond to analysis of an actuator with $\mathrm{n}$ stator cuts and $\mathrm{m}$ flotor cuts. Uncut actuator is included as a reference $(0 \mathrm{~s} 0 \mathrm{f})$. . . . . . . . .

3.18 Bandwidth for Case 1 with a single flotor cut and 1 to 16 stator cuts compared to Case 1 with the same number of cuts in the flotor and stator. . . . . . . . . . . . 57

4.1 System with state-dependent nonlinearity. . . . . . . . . . . . . . . . . . . . 59

4.2 System with state-dependent nonlinearity [7] . . . . . . . . . . . . . . . . . 60

4.3 Block diagram representation of single-sided non-laminated magnetic actuator with voltage

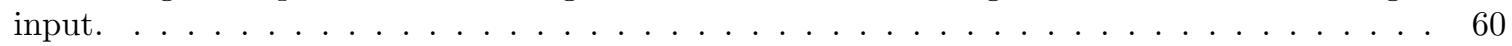

4.4 Non-laminated magnetic actuator with transconductance amplifier. . . . . . . . . . . . 61

4.5 Non-laminated magnetic actuator with amplifier in a separate inner feedback loop. . . . . . . 61

4.6 Nonlinear model with full Maxwell stress tensor approximated using average flux density. . . 62

$4.7 \quad F_{3}$ and total force, magnitude and phase, were determined by numerical integration or by an approximation using the average flux density. . . . . . . . . . . . 63

4.8 Nonlinear model with full Maxwell stress tensor. . . . . . . . . . . . . . . . . . . . . . 64 
4.9 Nonlinear model with full Maxwell stress tensor approximated using average perturbation flux density. . . . . . . . . . . . . . . . . . . . . . . . .

4.10 Complete nonlinear model, including eddy current effects, with with time-varying static reluctunce dependent on state. . . . . . . . . . . . . . . . . . . . . . . 65

4.11 Concise nonlinear model. . . . . . . . . . . . . . . . . . . . . . . . . . . . . 65

4.12 Feedback linearization for a single side of a thrust AMB . . . . . . . . . . . . . . . . 66

4.13 Block diagram representation of PID with pre-filter $F_{f}$. . . . . . . . . . . . . . . . . . . . 67

4.14 Step responses for reference and disturbance inputs $(1000 \mathrm{~N})$ based on linear analysis. . . . 76

4.15 Sensitivity frequency response (Sensitivity DC gain is 0 for both systems) . . . . . . . . . . 77

4.16 Complimentary sensitivity frequency response . . . . . . . . . . . . . . . . . . . . . 78

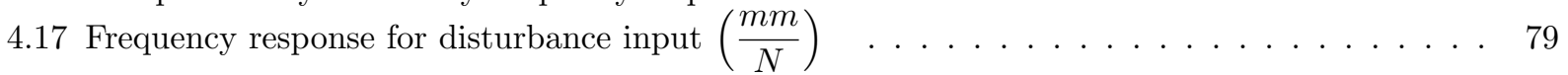

4.18 Loop gain frequency response (DC loop gain is infinite for both systems) . . . . . . . . . 80

4.19 Simulated reference tracking performance for a series of step inputs. Solid lines at $1 \mathrm{~mm}$ represent the boundary where the thrust disk contacts the stator. . . . . . . . . . . . . . . 80

4.20 Simulated sinusoidal reference tracking with input frequencies of 5 and $50 \mathrm{~Hz}$, small and large amplitude. . . . . . . . . . . . . . . . . . . . . . . 81

4.21 Simulated response to sinusoidal disturbance forces with frequencies of 5 and $50 \mathrm{~Hz}$. . . . 82

4.22 Simulated response to $1000 \mathrm{~N}$ disturbance step input. . . . . . . . . . . . . . . . . . . . . . 82

4.23 Recorded noise signal and simulated response to $1000 \mathrm{~N}$ disturbance step input with sensor noise added to position output signal. . . . . . . . . . . . . . . . . . . . . . . 83

4.24 Amplified noise and simulated response to $1000 \mathrm{~N}$ disturbance step input with amplified sensor noise added. . . . . . . . . . . . . . . . . . . . . . . . . . . 84

4.25 Frequency response for disturbance rejection while operating off-center. . . . . . . . . . . 85

4.26 Tracking performance of a sinusoidal reference while operating off-center. . . . . . . . . . . 85

4.27 Frequency response of FEA model, approximate analytic model and approximate analytic model with $c$ and $R^{0}$ optimized to fit FEA. . . . . . . . . . . . . . . . . . . . . 86

4.28 Monte Carlo analysis of robustness to model variation with a $1000 \mathrm{~N}$ step disturbance force for 1000 combinations of plant parameters. The disturbance response shows upper and lower bounds for each control type. Nominal disturbance response based on linear analysis is also shown. Plant model parameters were varied according to the distribution shown. . . . . . . .

4.29 Monte Carlo analysis for FBL-PID control of an idealized thrust AMB without eddy current effects and a typical thrust AMB with eddy current effects. The disturbance response shows upper and lower bounds for actuator. . . . . . . . . . . . . . . . . . 87

4.30 Frequency response for PID and FBL-PID control $\left(\frac{F}{m m}\right) \ldots \ldots$

4.31 Flux densities for $5 \mathrm{~A}$ current, static and $20 \mathrm{~Hz}$ perturbation. . . . . . . . . . . . . 88 


\section{Chapter 1}

\section{Introduction}

\subsection{Brief History of Magnetic Bearings}

Magnetic bearings were invented by experimental physicist Jesse Beams at the University of Virginia in the 1930's. Beams sought to develop a centrifuge with a rotational speed limited only by material strength [8]. Around this time, centrifuges had become useful in chemistry and biology for molecular sedimentation used to determine the molecular weight or size of large molecules. However, prior to 1930, centrifuges were limited, primarily by friction, to speeds of several thousand rpm. Heat induced by friction also led to convection currents and experimental error [4]. Beams design built on that of a previous design which involved a rotor suspended by and driven by compressed air [8]. To surpass speed limitations induced by friction, Beams used a vacuum chamber [8] and developed the first magnetic bearing, which supported the rotor axially, Figure 1.1 $[4,8]$.

The electromagnet used to suspend the rotor was placed outside the vacuum chamber and mounted in a flexible housing allowing it to track the whirl of the rotor [8]. A servo mechanism was used to stabilize the axial position of the rotor. This servo mechanism included a light which was shone horizontally across the top of the rotor onto a photoelectric cell which fed back to the suspension electromagnet [8]. Position sensing in later designs used a conducting loop above the rotor which acted as a variable resistor in response to rotor position [8]. In order to spin the rotor, torque was provided by an induction motor, which was also located outside the vacuum chamber $[4,8]$. This design met Beams's goal of reaching rotational speeds limited only by the component material strength, and thereby achieving a maximum rotational speed of $1.5 \times 10^{6} \mathrm{rps}$ which still holds as the fastest rotation speed ever achieved [5]. Peripheral speeds reached $1100 \mathrm{~m} / \mathrm{s}$. 


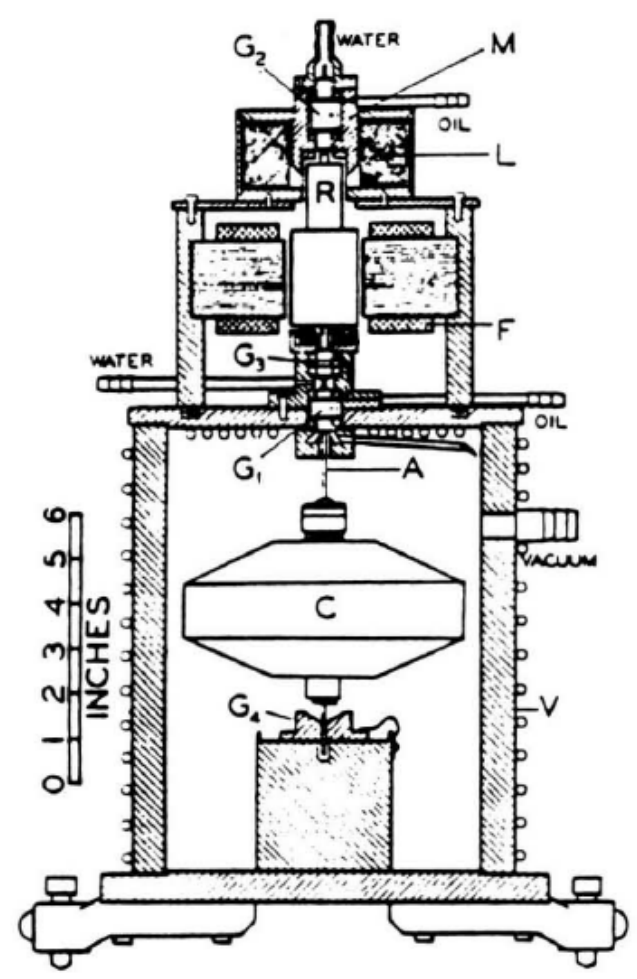

Figure 1.1: Drawing of Jesse Beams vacuum-type magnetically suspended ultracentrifuge. Shaft (A), rotor (C), induction motor $(\mathrm{F})$, suspension electromagnet $(\mathrm{M})$, magnetic suspension core (R), coil windings (L), oil gland $\left(\mathrm{G}_{1}\right)$, oil bearing $\left(\mathrm{G}_{2}\right.$ and $\left.\mathrm{G}_{3}\right)$, viscous damper $\left(\mathrm{G}_{4}\right)$, vacuum chamber $(\mathrm{V})$. Figure adapted from [4].

These new ultracentrifuge designs opened up new research opportunities in many fields such as strength of materials, where a material's strength could be analyzed by increasing the rotational speed until the material failed [8]. Biologists and chemists also benefited from the improved experimental accuracy of biochemical separation enabled by Beams's design. Beams also developed a method of separating isotopes via gas centrifugation that eventually became the preferred method of concentrating ${ }^{235} \mathrm{U}$ in the US [8]. Beams continued his work on magnetic suspension through the 1940's and 50's, focusing on levitation in a single plane with a constant gravity load [5]. Work on magnetic bearings was continued by other groups in the 1960's and 70's in the US, Japan, and France. However, the majority of this work focused on passive magnetic bearings [5]. It wasn't until the availability of inexpensive semiconductors capable of rapid switching of high currents in the 1970's that advancements in active magnetic bearing (AMB) technology began to accelerate $[5,9]$. Semiconductors have played a significant role in the progression of AMB technology, due to the fact that they are critical to the operation of power electronics as well as digital controllers. 


\subsection{Introduction to Active Magnetic Bearings}

Active magnetic bearings are currently used in a number of commercial applications including turbo-molecular pumps, energy storage flywheels, and compressors. In addition to axial support of the rotor weight, AMBs now support high speed rotors, subjected to large disturbance forces, both axially and radially. A typical configuration of a rotor supported by AMBs is shown in Figure 1.2, which shows an eight stage compressor supported by AMBs. Two radial AMBs, with two degrees of freedom each and one axial or thrust AMB with a single degree of freedom support the rotor without contact. Position sensors on each axis of the radial AMBs and an axial position sensor all feed their signals to a controller which in turn sends current reference signals to the amplifiers to drive the magnetic actuators which maintain a desired rotor position. Passive mechanical backup bearings, typically ball or roller element bearings, are used to minimize damage in case of any failure within the AMB system by preventing the rotor from contacting the magnetic actuators or other parts of the stator.

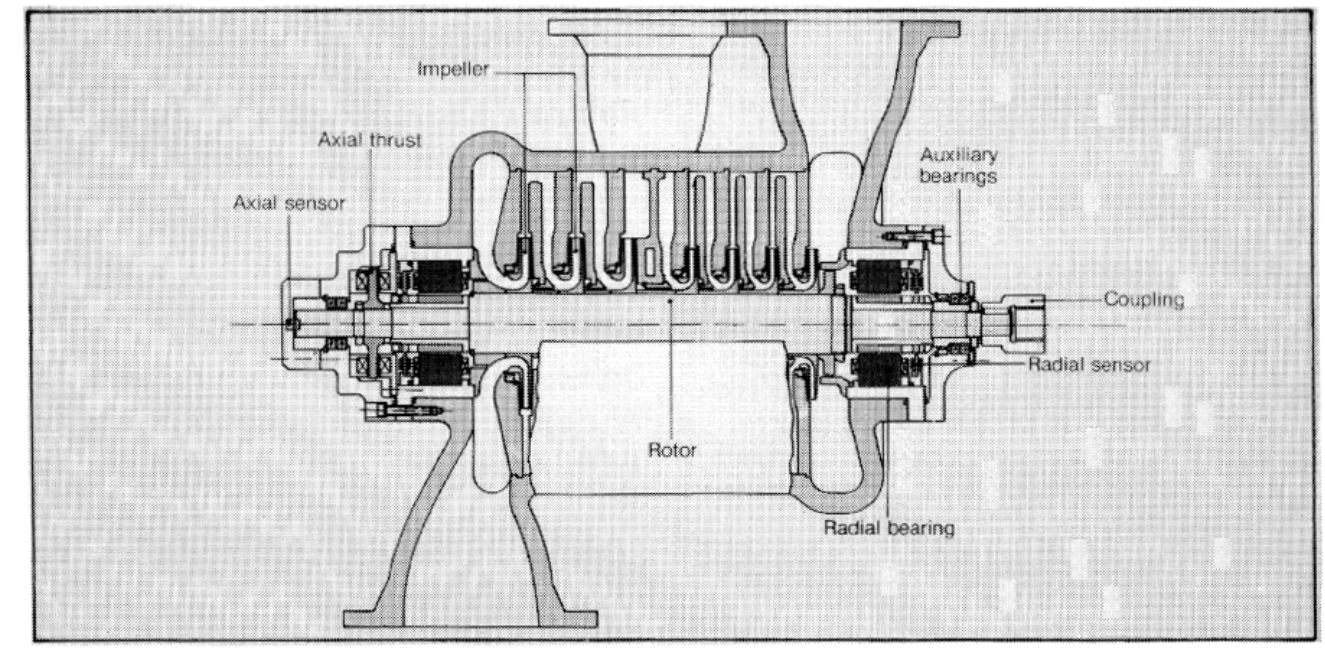

Figure 1.2: Cross section of an eight stage compressor supported by magnetic bearings. Figure adapted from [5].

Typically, AMBs are configured as opposing actuator pairs for each axis as depicted by the diagram of a thrust bearing in Figure 1.3. Magnetic thrust bearings tend to be axisymmetric such that in cross section each actuator resembles two C-type electromagnets. Viewed from the axial direction, thrust bearings look more like a disk with a groove containing an insulated coil. Each coil of the thrust bearing pair is driven by a separate power amplifier as shown in Figure 1.4. The entire thrust AMB system is also depicted in a simplified block diagram form in Figure 1.4. A rotor position signal from a sensor is routed through a signal conditioning circuit to filter noise and scale the signal, the position signal is then converted to a digital signal input to the controller which generates a desired coil current or voltage demand signal which is converted 
back to an analog signal for the power amplifiers. In current-mode, the amplifiers receive a reference signal corresponding to the desired coil currents. A feedback control loop internal to the amplifiers generates a voltage to match the current reference. The coils provide the magnetomotive force to generate flux in the electromagnetic actuator providing the force to stabilize the rotor.
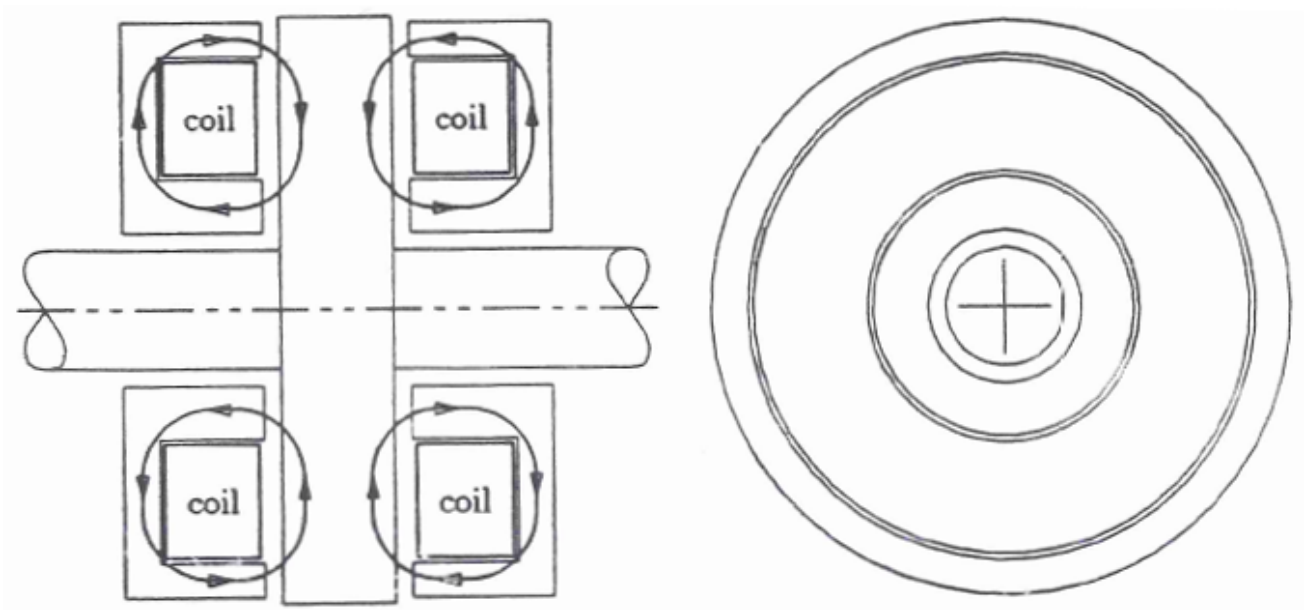

Figure 1.3: Thrust active magnetic bearing. Cross section (left) and axial view. Figure adapted from [6].

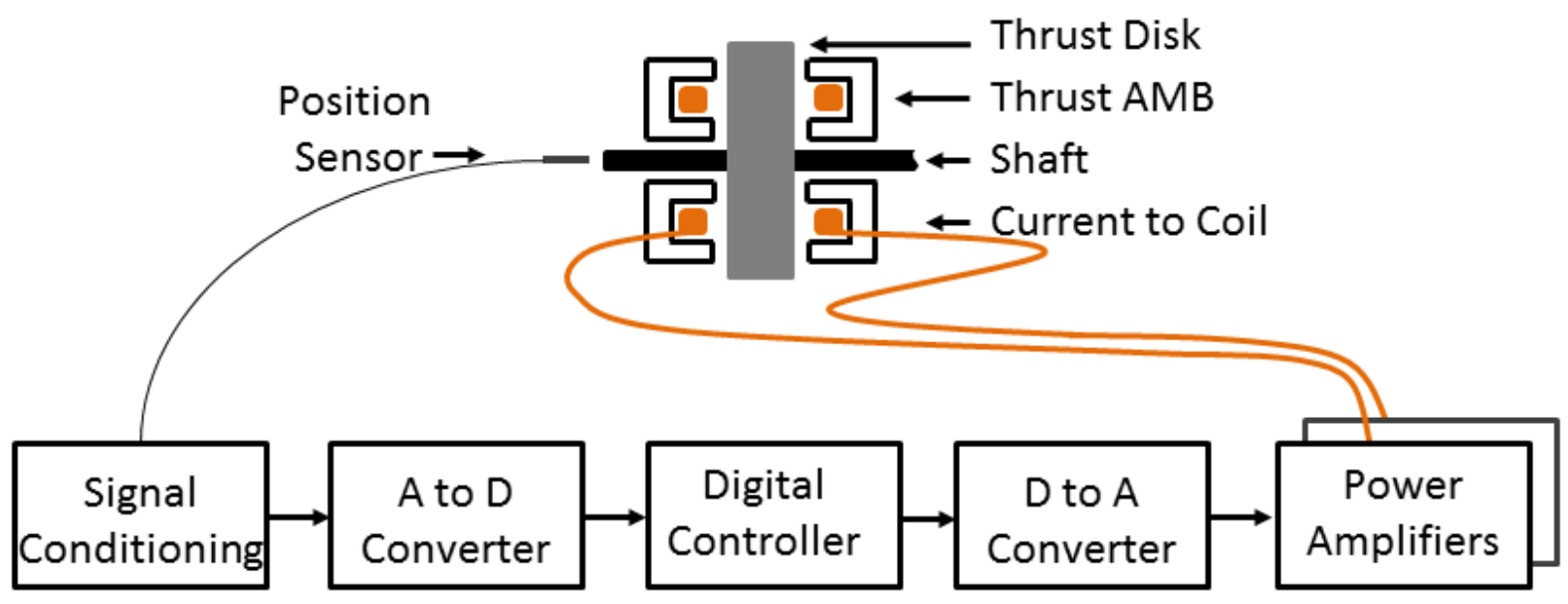

Figure 1.4: Thrust AMB system showing thrust disk portion of a rotor and the loop between rotor position and AMB including, axial position sensor, sensor signal conditioning, digital controller, power amplifier, and thrust AMB.

Thrust and radial AMBs are both made of ferromagnetic material, often silicon iron, and operate based on the same physical principles. However, there is one key difference between the two. Radial bearings are normally laminated while thrust AMBs are constructed of solid material. Laminated electromagnets are constructed of thin sheets of material that are stamped into shape and glued together as a stack as depicted in Figure 1.6 which shows two opposing quadrants of a laminated radial bearing. The laminations insulate 
the ferromagnetic material restricting the flux path to thin regions in an effort to make the flux flow more uniformly throughout the material. Electromagnetic interactions in solid material tend to generate eddy currents which reduce efficiency $[10,11,12,13,14,15,3,1,2]$. By using laminations in radial bearings much of the effects of eddy currents can be mitigated. In Figure 1.6 it can be seen that the natural flux path lies within the plane of the lamination layers. Unfortunately, thrust bearing geometry makes lamination much more complicated and expensive compared to radial AMBs.

Laminations for thrust magnetic bearings would have to be wedge shaped rather than flat sheets which would dramatically increase the cost of manufacturing. In addition, the large diameter typical of thrust disks requires high strength material to withstand the stress of high speed rotation, ruling out the possibility of a laminated thrust disk. While lamination of thrust AMBs is not practical, thrust stator segmentation has become more common recently and may be thought of as a crude form of lamination. Figure 1.5 shows a standard solid thrust AMB stator and a segmented thrust AMB. Separating the thrust stator serves the same purpose as laminations: breaking up the eddy current paths into smaller loops.

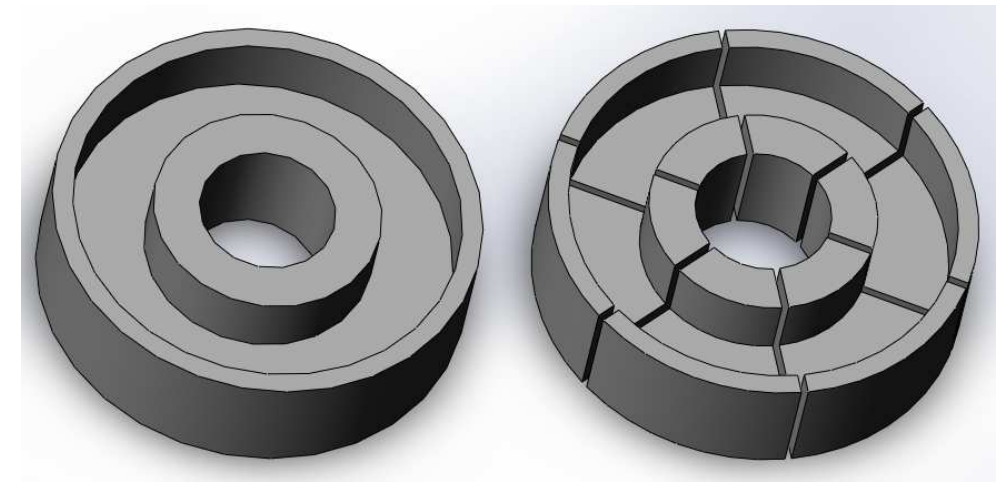

Figure 1.5: Depiction of a traditional thrust stator and a segmented stator (right).

Without laminations, eddy current effects make control of axial vibrations a challenging problem for applications with large, high frequency, axial disturbance forces. High speed compressors are often plagued by axial disturbance forces outside the bandwidth of the thrust AMB. In an effort to improve efficiency, compressors are operating at higher pressures and at the same time, magnetic bearings are gaining market share. With the higher operating pressure, larger disturbance forces are generated in the axial direction as a result of emergency shut down, overpressure release valve activation, and compressor surge. Most research in the design and control of AMBs for rotating machinery has focused on radial magnetic bearings which are generally laminated in order to minimize eddy current effects. Because non-laminated thrust AMBs suffer from reduced bandwidth as a result of eddy current effects, they tend to be a performance limiting component for many applications, therefore opening up the possibility for dramatic improvements in machine performance. 


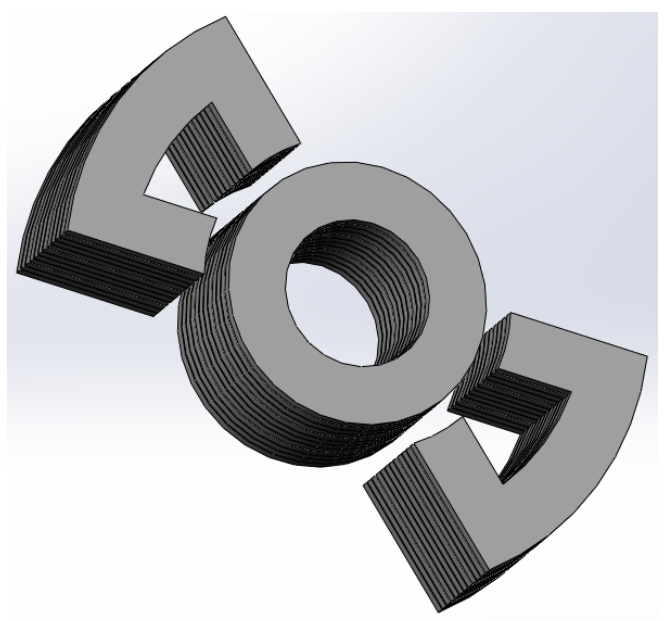

Figure 1.6: Depiction of a single axis of a laminated radial active magnetic bearing.

\subsection{Analytic Modeling of Non-laminated Magnetic Actuators}

Analytic models for non-laminated magnetic actuators have been developed by several groups. Zmood et al. developed the first model for a non-laminated C-type actuator, including eddy current effects, in 1987[13]. Zmood's model was for long C-type actuators where the length is much greater than the pole width such that eddy current effects in the ends may be neglected. This assumption simplified the differential equation to a single dimensional diffusion equation and built on the work of Stoll describing the effect of eddy currents in a solid plate [12]. Zmood et al. developed a transcendental model for total flux with respect to coil current and for perturbation force with respect to coil current and position [13]. However, in addition to the assumption that the actuator is long, flux density was assumed to follow a distribution in the air gap similar to that in the iron, which has been shown not to be the case, as will be discussed in Chapter 2 .

Feeley developed an analytic model for C-type magnetic actuators including eddy current effects at the actuator ends [15]. The analytic model for flux with respect to coil current was more elaborate than Zmood's and also included transcendental functions. Using an ad hoc approximation, a rational half-order model more suitable for control design was developed [15]. This is the first appearance of a half-order model for non-laminated actuators with eddy current effects. Feeley's model is more versatile than Zmood's but also failed to capture the difference in flux distribution in the air gap versus the iron that results from eddy current effects.

Kucera and Ahrens developed a model for non-laminated axisymmetric geometries with eddy current effects [16]. The geometry was divided into regions and a relationship for coil current to flux was determined using Maxwell's equations. The analytic model was formed by combining the general models for each region. 
Similar to the previous works cited, the flux density in the air gap was assumed to be homogeneous, which is not the case in the presence of eddy currents.

Building on the work of Zmood, Meeker et al. developed a model for laminated AMBs that included eddy current effects [14]. A magnetic circuit model was used to develop an analytic model that included flux leakage and fringing as well as eddy current effects for radial AMBs. A 1-D model for eddy current effects was combined with a magnetic circuit model of the actuator lamination network, and flux leakage and fringing paths. Finite element modeling with a static coil current was used to estimate the reluctance in the fringing and leakage paths.

More recently, Zhu et al. developed extremely accurate analytic models for axisymmetric [3] and C-type [1] electromagnetic actuators. Recognizing that flux density in the air gap is not homogeneous but rather follows a frequency dependent distribution, Zhu was able to capture this effect using magnetic circuit analysis and the idea of skin effect leading to an effective reluctance $[3,1,2]$. Axisymmetric and C-type geometries were divided into regions and effective reluctance networks were developed for each region. Frequency dependent reluctance for each region was modeled relying on the well known skin effect to determine an effective reluctance for each element in the network. The analytic models developed by Zhu et al. were the first to accurately capture the frequency dependent distribution of flux density in the air gap. Zhu's model is the starting point for the work described here and will be covered in detail in Chapters 2 and 3 .

Following up on Zhu's axisymmetric model, Sun et al. developed an analytic model for axisymmetric AMBs including flux leakage and fringing . Extensive finite element modeling was done in ANSYS for a sinusoidal current input or sinusoidal displacement using mesh morphing in the air gap elements. These techniques allowed them to compare the frequency response for position versus current inputs which were shown to be similar. Sun et al. also validated their analytic model with experimental results.

\subsection{Fractional-Order Differential Equations}

Beginning with Feeley's development of a fractional-order model for C-type actuators [15], electromagnetic actuator models including eddy current effects have all been fractional-order. The idea of fractional calculus has been around since L'Hôpital, when in 1695 he pondered the idea in a letter to Leibniz [17]. In fractional calculus the order of differentiation is generalized to include non-integers such that for

$$
D^{n} f(t)=\frac{d^{n} f(t)}{d t^{n}}
$$

$\mathrm{n}$ may be any real number with $n>0$ representing differentiation and $n<0$ representing integration [18]. 
Fractional order modeling and control have become more common recently as tools for systems with memory and complex systems of infinite order such as transmission line systems, piezoelectrics, and of course electromagnetic systems with eddy current losses [18]. Viscoelasticity provides an example of system memory where strain is time-dependent and effective stiffness depends on the rate of change in the disturbance force. The ability of fractional derivatives to model system memory can be seen in the following form of the Grünwald-Letnikov definition of a fractional derivative of order $\alpha$ given by

$$
D^{\alpha} f(t)=\sum_{k=0}^{m} \frac{f^{(k)}\left(0^{+}\right) t^{k-\alpha}}{\Gamma(m+1-\alpha)}+\frac{1}{\Gamma(m+1-\alpha)} \int_{0}^{t}(t-\tau)^{m-\alpha} f^{(m+1)}(\tau) \mathrm{d} \tau
$$

[18], where $\alpha \in R^{+}, m>\alpha-1$, and $\Gamma(\cdot)$ represents the gamma function defined by

$$
\Gamma(n)=\int_{0}^{\infty} x^{n-1} e^{-x} \mathrm{~d} x
$$

As can be seen in Equation 1.2, fractional derivations have properties of both integer order differentiation and integration. A fractional integral may be defined by the Reimann-Liouville definition given by

$$
D^{-n} f(t)=\frac{1}{\Gamma(n)} \int_{0}^{t} f(y)(t-y)^{n-1} \mathrm{~d} y
$$

where $n \in R^{+}[18]$.

Fractional order systems that are continuous-time may be represented in transfer function form by

$$
G(s)=\frac{Y(s)}{U(s)}=\frac{b_{m} s^{\beta_{m}}+b_{m-1} s^{\beta_{m-1}}+\cdots+b_{0} s^{\beta_{0}}}{a_{n} s^{\alpha_{n}}+a_{n-1} s^{\alpha_{n-1}}+\cdots+a_{0} s^{\alpha_{0}}}
$$

[18], where $\beta_{i} \in R$ and $\alpha \in R$, as opposed to integer order systems were $\beta_{i}$ and $\alpha_{i}$ are restricted to integer values.

Computational tools for analysis and simulation of fractional order systems are available as Matlab toolboxes. For example, CRONE, Ninteger, and FOMCON include many of the standard matlab functions and tools for approximating fractional order transfer functions as integer order [17]. FOMCON is the primary tool used here for analysis of fractional order systems. 


\subsection{Segmentation of Thrust AMBs to Enhance Dynamic Perfor- mance}

Active magnetic bearings (AMBs) for axial loads are often a performance limiting component in rotating machinery such as compressors and machine tool spindles. In an effort to improve efficiency, compressors are being operated at higher pressures and magnetic bearings are gaining market share in this application space. AMBs allow contact free rotor support without lubrication or wear, and reduce energy losses 5- to 20-fold below conventional ball or fluid bearings [11]. With higher operating pressure, larger axial disturbance forces are being generated as a result of emergency shut down, over-pressure release valves, and compressor surge. Most research in the design and control of AMBs for rotating machinery has focused on radial magnetic bearings which are generally laminated in order to minimize eddy current effects. Design and control of thrust AMBs has not been nearly as prominent in the literature. However, because thrust magnetic bearings are traditionally non-laminated, eddy current effects make control of thrust AMBs challenging.

Powder metals, or soft magnetic composites, are one option for reducing eddy current effects. However, powder metallurgy introduces another set of deficiencies. Poor mechanical strength of powder metals precludes its use in thrust disks which often encounter the highest stress as they tend to be the largest diameter component of the rotor. Reduced permeability and low saturation flux density compared to the base material are also a deficiency of magnetic composites which can limit overall actuator performance. Powder metals may be a good option for some, but not all, applications. High speed or a requirement for high force density would preclude the use of magnetic composites. Therefore, the focus of this work is on stator segmentation and feedback linearization (FBL) as methods for improving dynamic performance of thrust AMBs.

A transformer with a single secondary loop (Figure 1.7) provides a good analogy for the eddy current effect in solid axisymmetric thrust AMBs. In the primary coil an oscillating voltage and current generates an oscillating magnetic flux. In this example the transformer material is ideal and does not conduct electrical current which means that no eddy currents exist in the iron. Electromagnetic induction, as a result of the magnetic flux, produces a voltage $\left(v_{e}\right)$ in the secondary coil. With an impedance load in the loop $\left(z_{e}\right)$, a current $\left(i_{e}\right)$ is also produced. The single secondary loop is analogous to the conducting loop of the iron in an axisymmetric AMB. The current generated in the secondary loop in the transformer induces a magnetomotive force in opposition to that of the primary coil. This reduces the effective magnetomotive force, analogous to back EMF in a DC motor. If the secondary loop in the ideal transformer were replaced with smaller diameter loops, in parallel, placed adjacent to the iron, rather than circumscribing the iron, then the eddy current effect may not be as severe. This is the idea behind segmentation of a thrust AMB stator. 


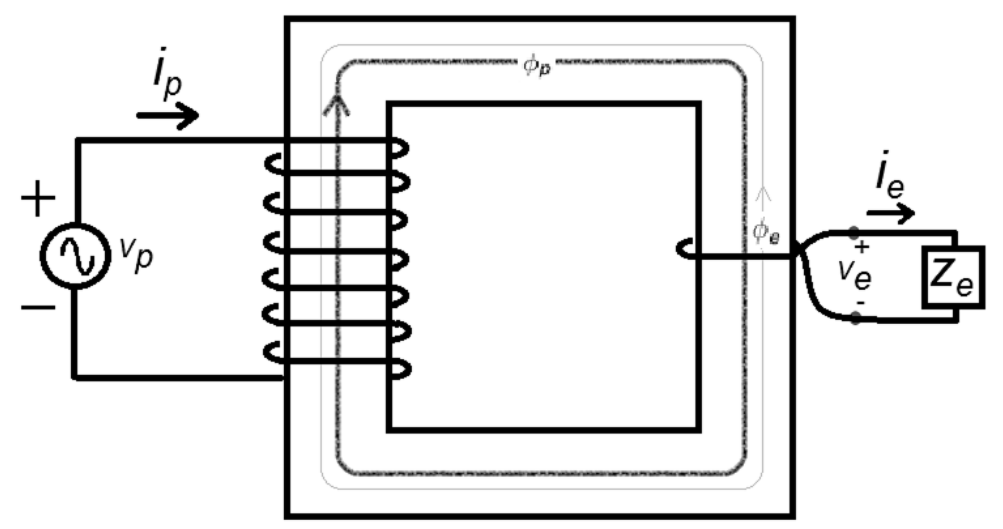

Figure 1.7: Ideal transformer with a single secondary loop.

Segmented stators (Figure 1.5 (right)) are a promising method to mitigate parasitic losses associated with eddy currents and improve dynamic performance of non-laminated thrust bearings. Stator segmentation serves the same purpose as laminations and could be considered a coarse lamination strategy. By impeding the eddy current loop around the stator periphery with a single cut, or further breaking up the eddy current paths by introducing more stator segments, the geometry approaches that of a laminated thrust bearing. However, the practical size of these segments is constrained such that eddy current effects cannot be ignored in segmented thrust bearing models as they can be for fully laminated radial bearings. The thrust disk, or flotor, must be solid to maintain its structural integrity. While segmented stator designs are currently in use, a systematic study of the effect on dynamic performance has not been published nor is an analytic model for this type of geometry currently available. Without an analytical model it is difficult to determine whether segmentation will yield adequate improvements for a given application.

\subsection{Feedback Linearization}

Most control implementations are designed for linear systems while most systems are nonlinear. Typically a nonlinear plant model is linearized about a desired operating point. As the operating conditions deviate from this point, the closed-loop performance degrades. Gain scheduling is a simple and common solution where a series of controllers are designed over a range of operating conditions and time-varying gains are determined by interpolation. This strategy is difficult to verify as simulation performance does not always translate to performance in implementation [19]. Feedback linearization (FBL) is an alternative strategy that avoids these problems.

FBL, in its simplest form, is simply an inversion of the plant which cancels nonlinearities. For example, for a plant model where the input enters through $f(\cdot)$, input $u$ may be chosen as $u=f^{-1}(v)$ where $v$ is a 
pseudo-input [19], Figure 1.8. FBL does not stabilize or control a system but does linearize the system so that a single controller may yield consistent performance over a range of conditions. Feedback linearization has been proven useful in magnetic bearings by several groups. Trumper et al. used a one degree of freedom magnetic suspension system to achieve consistent control performance across the entire operating range [20]. Lindlau et al. showed that FBL may be combined with $\mu$ control to guarantee performance over a wide operating range for a single degree of freedom magnetic bearing test rig operated in voltage mode [21, 22]. Chen et al. showed similar results for a radial AMB operated in current mode [7].

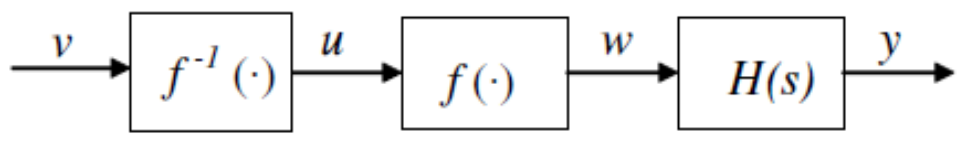

Figure 1.8: Basic example of feedback linearization.

\subsection{Overview}

In an effort to improve performance of non-laminated thrust AMBs this work focuses on both modeling and control. Current analytic models for axisymmetric magnetic actuators developed by Zhu et al. [3, 1, 2] do not cover geometries with a center hole which are typical for thrust AMBs used in rotating machinery. In Chapter 2, Zhu's model for non-laminated magnetic actuators is extended to include geometries with a center hole. The new model is validated by comparing the frequency response to results from finite element analysis.

Segmented stators have become more common recently in non-laminated thrust bearings. Based on analytic models of other actuator geometries, disrupting the eddy current path around the entire stator should reduce eddy current losses. However, a systematic analysis of the effect of stator segmentation has yet to be published in the literature. Stator segmentation disrupts the axial symmetry of thrust AMBs and shifts the geometry towards that of a C-type magnetic actuator. Building again on a model developed by Zhu et al. in $[1,2]$, an analytic model for segmented thrust AMBs is developed in Chapter 3 and verified by FEA. Based on the analytic model and extensive FEA modeling, it is shown that for most geometries, division of the stator into six segments can approximately double the bandwidth.

Accurate analytic models will aid in the design and control of non-laminated thrust AMBs. A good analytic model can also be used to improve performance of existing machines by improving control. The ability to adjust performance by changing software rather than hardware is another advantage of AMBs. In chapter $4 \mathrm{FBL}$ is explored as a way to reduce the nonlinearities in performance that are inherent to magnetic actuators. FBL has been shown to improve performance consistency for laminated magnetic bearings but 
has not previously been attempted for non-lamainted AMBs. The feedback linearization method presented in Chapter 4 compensates for position nonlinearities as well as dynamic effects due eddy currents. The combination of dynamic and nonlinear compensation is shown in simulation analysis to improve reference tracking and disturbance rejection without any loss in robustness or significant sensitivity to noise, both of which are typical concerns when implementing FBL. 


\section{Chapter 2}

\section{Analytic Model for Non-laminated}

\section{Thrust AMBs}

Modeling eddy current effects in solid magnetic actuators has traditionally required time consuming finite element analysis (FEA). Revising a finite element model can be tedious, and it requires even more effort for design optimization. Once the design phase is complete, controller design for thrust AMBs is normally done by trial and error or by using traditional magnetic circuit models that neglect eddy current effects, along with experimental identification of actuator parameters. Development of an accurate analytic model for non-laminated electromagnetic actuators would save time and greatly improve the process of design and control of non-laminated AMBs. This was the motivation for the work by Zhu et al. to develop an analytic model for solid magnetic actuators $[3,1,2]$. The analytic model they developed is a dramatic improvement over previous models accounting for eddy current effects but does not allow for axisymmetric geometries with a center hole which is typical of thrust bearings in rotating machines. In this chapter the analytic model developed by Zhu et al. is extended to include geometries with a center hole. Zhu's model, and the extension developed here, are for linear isotropic materials and do not account for leakage, fringing, hysteresis, or flux saturation $[3,1,2]$.

\subsection{Current Model for Axisymmetric Actuators}

Early models of solid magnetic actuators assumed that flux density in the air gap was homogeneous. However, due to eddy current effects, the flux density in solid actuators varies across the air gap. For an axisymmetric geometry with cross section shown in Figure 2.1, the axis of symmetry is the z-axis, flux density varies with 
radial position along the air gap. Figure 2.2 shows flux density in the air gap predicted by FEA beginning at the central axis, $r=0$ and moving out towards the outer radius, $r=r_{3}$. Moving towards the outer radius across the inner pole, flux density increases. Flux fringing and leakage across the coil was neglected in this analysis so the flux density in the coil groove, $r_{1}<r<r_{2}$, is zero. Moving out across the outer pole, $r_{2}<r<r_{3}$, flux density decreases.

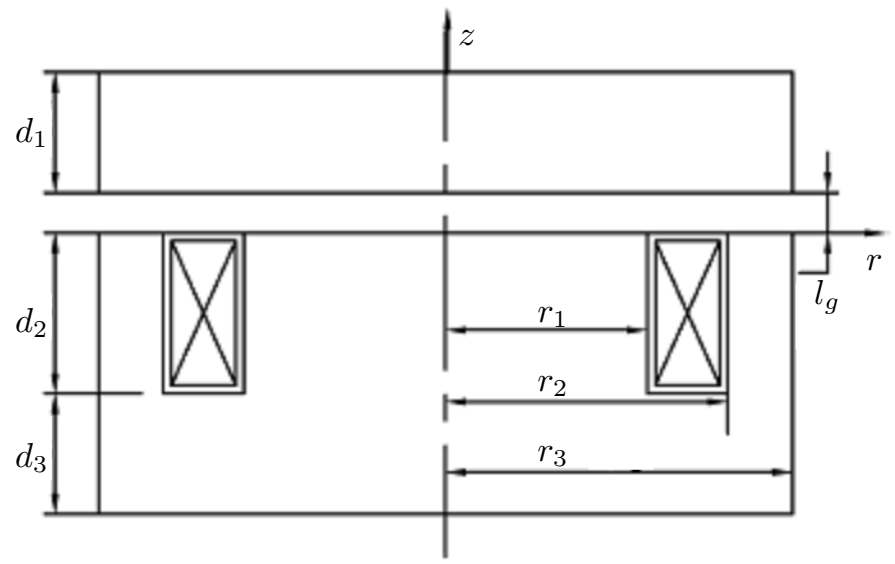

Figure 2.1: Parameters describing axisymmetric actuator geometry without a center hole.Adapted from [3].

Flux Density Across Air Gap

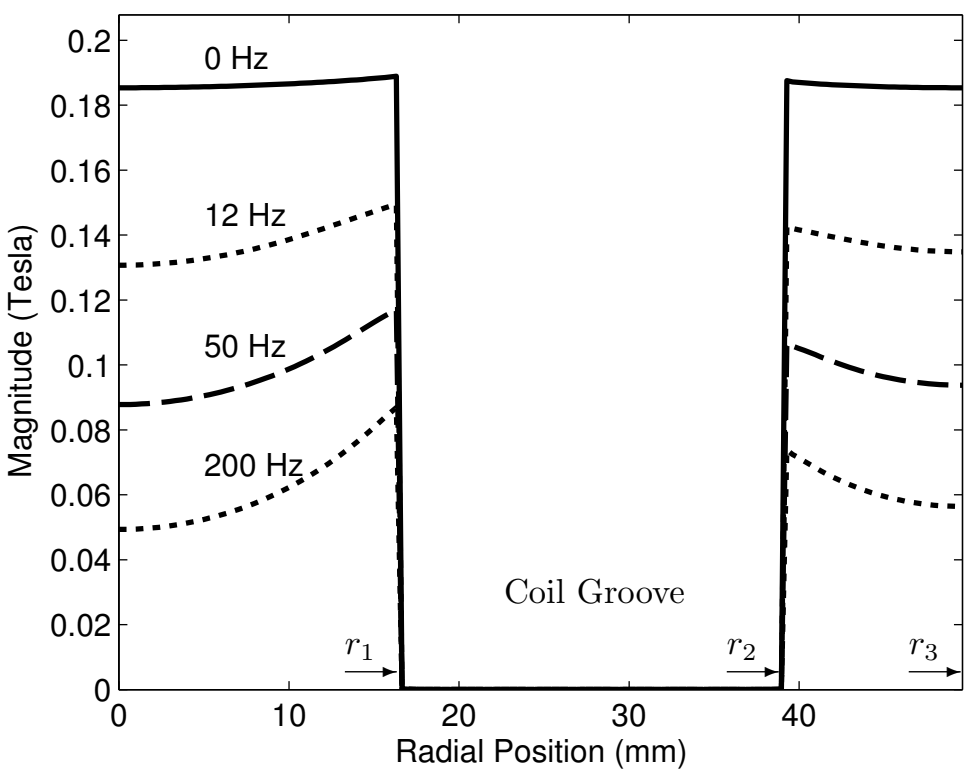

Figure 2.2: Air gap flux density varies with radial position.

Zhu et al. developed an extensive 2-D magnetic circuit model of non-laminated axisymmetric actuators, based on the ideas of skin effect and effective reluctance, that is able to capture this variation in air gap flux density. Eddy current-induced skin effect is a well studied phenomenon, resulting from eddy currents, 
describing the tendency for electromagnetic waves to travel near the surface of solid conductors. The depth at which flux amplitude is reduced by $1 / e \approx 1 / 3$ is the skin depth and is given by $\delta=1 / \sqrt{\pi f \mu_{r} \mu_{0} \sigma}[10,3]$. Neglecting the effects of eddy currents, the reluctance along a solid uniform material of length $l$ and cross sectional area $A$ is given by

$$
R=\frac{l}{\mu_{r} \mu_{0} A},
$$

where $\mu_{r}$ is the magnetic permeability of the material relative to that of free space, given by $\mu_{0}$. However, in a solid magnetic actuator with eddy current effects, the effective cross-sectional area permeable to flux is reduced with increasing frequency, consistent with the skin effect, giving rise to the idea of effective permeability and effective reluctance $[3,2]$. Using the idea of skin effect to develop a magnetic circuit model with reluctance elements dependent on frequency, i.e. effective reluctance, allowed Zhu to accurately capture the effects of eddy currents with a simple 2-D analytic model that agrees closely with FEA.

Figures 2.3 and 2.4 show the flux contours from FEA, using Finite Element Method Magnetics (FEMM) [23], for a static excitation current and a harmonic excitation current of $50 \mathrm{~Hz}$. The flux contours show that the flux is generally flowing parallel to the air gaps or vertically in the z-axis. This key observation allowed Zhu et al. to divide the geometry in such a way that a series of 1-D magnetic circuits would accurately model the effective reluctance of the actuator [3, 2]. Figure 2.1 shows how the axisymmetric geometry was defined using polar coordinates in $[3,1,2]$. The geometry was then divided into six regions as shown in Figure 2.5. The regions were chosen based on the flux contours mentioned above. Region 1 includes the inner air gap, the upper portion of the stator and the inner portion of the floater. To get an idea of how the effective reluctance for each region was determined, Figure 2.6 shows the reluctance network for Region 1, developed by Zhu et al. in [2], which includes the details of the derivation of effective reluctances for each region. In the figure it can be seen that the flux path originating below the air gap, with magnetomotive force $\left(F_{M}\right)$, flows mostly parallel to the air gap within the stator. Crossing the air gap the flux is perpendicular to the material surface flowing in the $\mathrm{z}$ direction. The path across the air gap is modeled as an infinite number of reluctance elements in a parallel circuit. In the floater, flux once again flows predominantly in the radial direction parallel to the air gap surface. Regions 1 and 3 have similar reluctance networks and are more complicated than the other regions because they include the air gap. Regions 2 and 5 have similar reluctance networks with flux flowing predominantly in the radial direction. Regions 4 and 6 also have similar reluctance networks with flux flowing primarily parallel to the z-axis. Reluctance networks for each of these regions are covered in detail in [2]. 


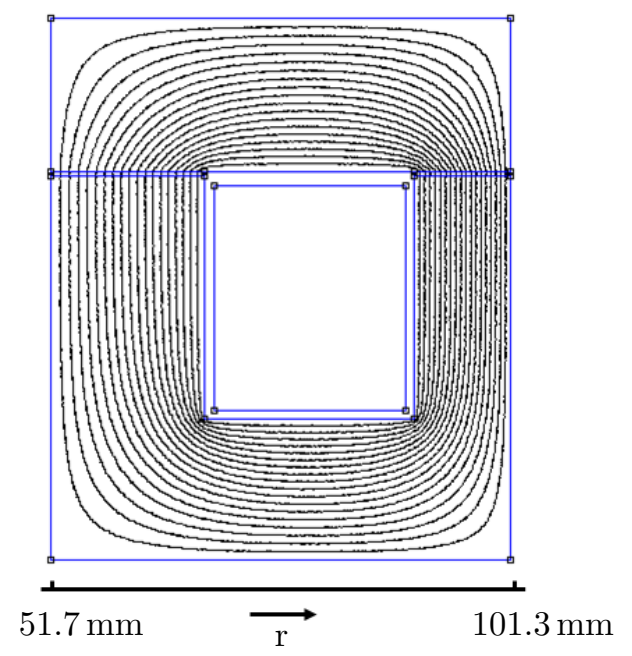

Figure 2.3: Flux contours of geometry with a center hole with a static current excitation.


Figure 2.4: Flux contours real (left) and imaginary (right) from FEA with a $50 \mathrm{~Hz}$ excitation current.

\subsection{Model for Axisymmetric Actuators With a Center Hole}

Thrust bearings for rotating machinery typically have a center hole to accommodate a shaft. Zhu's model does not allow for geometries with a center hole. Of the six regions in Zhu's axisymmetric model, Regions 1 and 6, border the central axis or center hole. To accommodate geometries with a center hole, effective reluctance models of these two regions must be modified. To describe the geometry containing a center hole a new parameter $\left(r_{0}\right)$ representing the radius of the center hole is introduced.

Following the procedure used by Zhu, [3, 2], the effective reluctance for Region 1 with a center hole is developed first. From the reluctance network, Figure 2.6, and circuit theory it can be seen that the magnetomotive force at a radial position in the floater, $F_{M_{f l}}(r)$, is given by the total magnetomotive force, 


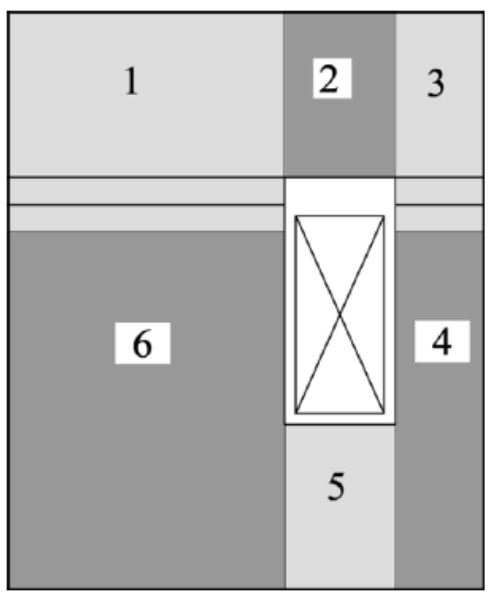

Figure 2.5: Axisymmetric geometry divided into six regions, each with an effective reluctance dependent on frequency.

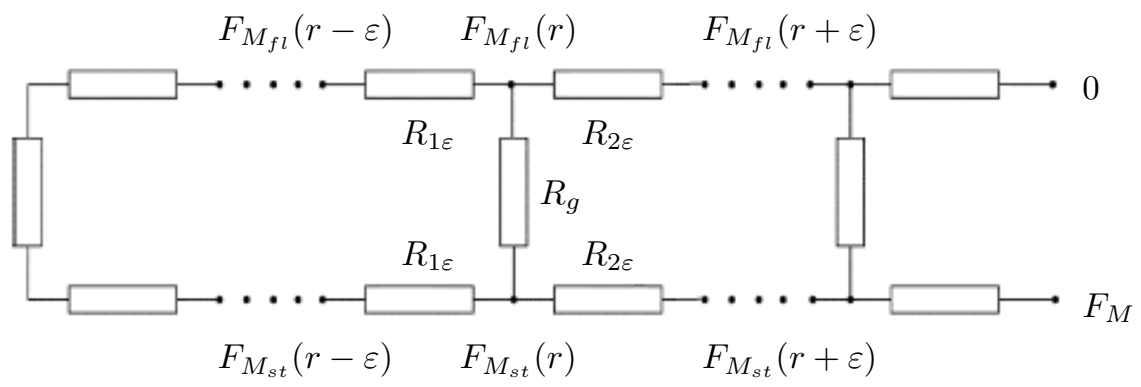

Figure 2.6: Zhu's reluctance network for Region 1.

$F_{M}$, minus the loss in magnetomotive force along the stator such that

$$
F_{M_{f l}}(r)=F_{M}-F_{M_{s t}}(r),
$$

where $F_{M}$ is the magnetomotive force equal to the number of coil turns $(N)$ times the coil current $(i)$. From the reluctance network for Region 1, Zhu et al. developed the following ordinary differential equation [2]:

$$
\frac{d^{2} F_{M_{s t}}(r)}{d r^{2}}+\frac{1}{r} \frac{d F_{M_{s t}}(r)}{d r}-\alpha_{1}^{2} F_{M_{s t}}(r)=-\frac{\alpha_{1}^{2}}{2} F_{M},
$$

where $\alpha=\sqrt{s \sigma \mu_{r} \mu_{0}}$ and $\alpha_{1}=\sqrt{\frac{2 \alpha}{\mu_{r} l_{g}}}$. Equation 2.3 is a modified Bessel equation with the complete solution shown in 2.4 below [2],

$$
F_{M_{s t}}(r)=\frac{F_{M}}{2}+C_{1} I_{0}\left(\alpha_{1} r\right)+C_{2} K_{0}\left(\alpha_{1} r\right)
$$


where $I_{n}(\cdot)$ is the nth-order modified Bessel function of the first kind and $K_{n}(\cdot)$ is the nth-order modified Bessel function of the second kind. Substitution of 2.2 into 2.4 also gives

$$
F_{M_{f l}}(r)=\frac{F_{M}}{2}-C_{1} I_{0}\left(\alpha_{1} r\right)-C_{2} K_{0}\left(\alpha_{1} r\right)
$$

For small $q, K_{0}(q) \approx \log \frac{2}{q}[2]$. Therefore, for $q \rightarrow 0,\left|K_{0}(q)\right| \rightarrow \infty$ and thus for $r_{0}=0, C_{2}=0$. However, for $r_{0}>0$, i.e. a geometry with a center hole, the values of constants $C_{1}$ and $C_{2}$ must be re-evaluated, resulting in:

$$
\begin{aligned}
C_{1} & =\frac{1}{2} \frac{F_{M} K_{1}\left(\alpha_{1} r_{0}\right)}{I_{1}\left(\alpha_{1} r_{0}\right) K_{0}\left(\alpha_{1} r_{1}\right)+I_{0}\left(\alpha_{1} r_{1}\right) K_{1}\left(\alpha_{1} r_{0}\right)} \\
C_{2} & =\frac{1}{2} \frac{F_{M} I_{1}\left(\alpha_{1} r_{0}\right)}{I_{1}\left(\alpha_{1} r_{0}\right) K_{0}\left(\alpha_{1} r_{1}\right)+I_{0}\left(\alpha_{1} r_{1}\right) K_{1}\left(\alpha_{1} r_{0}\right)}
\end{aligned}
$$

in this case. Substituting Equation 2.6 into Equations 2.4 and 2.5 results in two equations for magnetomotive force at the surface of the stator, $F_{M_{s t}}$, and floater, $F_{M_{f l}}$, in the air gap as a function of radial position $r$ :

$$
\begin{aligned}
& F_{M_{s t}}(r)=\frac{F_{M}}{2}+\frac{F_{M}}{2} \frac{I_{0}\left(\alpha_{1} r\right) K_{1}\left(\alpha_{1} r_{0}\right)}{I_{1}\left(\alpha_{1} r_{0}\right) K_{0}\left(\alpha_{1} r_{1}\right)+I_{0}\left(\alpha_{1} r_{1}\right) K_{1}\left(\alpha_{1} r_{0}\right)}+\frac{F_{M}}{2} \frac{K_{0}\left(\alpha_{1} r\right) I_{1}\left(\alpha_{1} r_{0}\right)}{I_{1}\left(\alpha_{1} r_{0}\right) K_{0}\left(\alpha_{1} r_{1}\right)+I_{0}\left(\alpha_{1} r_{1}\right) K_{1}\left(\alpha_{1} r_{0}\right)} \\
& F_{M_{f l}}(r)=\frac{F_{M}}{2}-\frac{F_{M}}{2} \frac{I_{0}\left(\alpha_{1} r\right) K_{1}\left(\alpha_{1} r_{0}\right)}{I_{1}\left(\alpha_{1} r_{0}\right) K_{0}\left(\alpha_{1} r_{1}\right)+I_{0}\left(\alpha_{1} r_{1}\right) K_{1}\left(\alpha_{1} r_{0}\right)}-\frac{F_{M}}{2} \frac{K_{0}\left(\alpha_{1} r\right) I_{1}\left(\alpha_{1} r_{0}\right)}{I_{1}\left(\alpha_{1} r_{0}\right) K_{0}\left(\alpha_{1} r_{1}\right)+I_{0}\left(\alpha_{1} r_{1}\right) K_{1}\left(\alpha_{1} r_{0}\right)}
\end{aligned}
$$

The flux density in the air gap $B_{g}(r)=\frac{\mu_{0}}{l_{g}}\left(F_{M_{s t}}(r)-F_{M_{f l}}(r)\right)[2]$ may then be evaluated as:

$$
B_{g}(r)=\frac{\mu_{0} F_{M}}{l_{g}}\left[\frac{K_{1}\left(\alpha_{1} r_{0}\right) I_{0}\left(\alpha_{1} r\right)+I_{1}\left(\alpha_{1} r_{0}\right) K_{0}\left(\alpha_{1} r\right)}{I_{1}\left(\alpha_{1} r_{0}\right) K_{0}\left(\alpha_{1} r_{1}\right)+I_{0}\left(\alpha_{1} r_{1}\right) K_{1}\left(\alpha_{1} r_{0}\right)}\right] .
$$

Solving for the total flux in the air gap, for Region 1, is achieved by integrating $B_{g}(r)$ from $r_{0}$ to $r_{1}$ :

$$
\phi_{g}=\int_{r_{0}}^{r_{1}} B_{g}(r) \cdot 2 \pi r \mathrm{~d} r=\frac{2 \pi \mu_{0} r_{1} F_{M}}{l_{g} \alpha_{1}}\left[\frac{I_{1}\left(\alpha_{1} r_{1}\right) K_{1}\left(\alpha_{1} r_{0}\right)-I_{1}\left(\alpha_{1} r_{0}\right) K_{1}\left(\alpha_{1} r_{1}\right)}{I_{1}\left(\alpha_{1} r_{0}\right) K_{0}\left(\alpha_{1} r_{1}\right)+I_{0}\left(\alpha_{1} r_{1}\right) K_{1}\left(\alpha_{1} r_{0}\right)}\right]
$$

Utilizing the relationship between magnetic flux in the air gap $(\phi)$, magnetomotive force $\left(F_{M}\right)$, and reluctance $(R)$ given by:

$$
\phi=\frac{F_{M}}{R}
$$


the effective reluctance for Region 1 can be expressed as:

$$
R_{1}(s)=\frac{l_{g} \alpha_{1}}{r_{1} 2 \pi \mu_{0}} \frac{I_{1}\left(\alpha_{1} r_{0}\right) K_{0}\left(\alpha_{1} r_{1}\right)+I_{0}\left(\alpha_{1} r_{1}\right) K_{1}\left(\alpha_{1} r_{0}\right)}{I_{1}\left(\alpha_{1} r_{1}\right) K_{1}\left(\alpha_{1} r_{0}\right)-I_{1}\left(\alpha_{1} r_{0}\right) K_{1}\left(\alpha_{1} r_{1}\right)}
$$

For the static case, the absence of eddy currents suggests that the reluctance would be determined by the air gap only, and indeed the result obtained by taking the limit of $R_{1}(s)$ as the complex variable $s$ approaches zero yields

$$
R_{1}^{0}=\lim _{s \rightarrow 0} R_{1}(s)=\frac{l_{g}}{\pi \mu_{0}\left(r_{1}^{2}-r_{0}^{2}\right)}
$$

which is the expected result. In addition, for $r_{0}=0$, the solution matches that obtained by Zhu et al. for the static reluctance for Region 1.

The development of the effective reluctance model for Region 6 with $r_{0}>0$ is slightly different than that for Region 1. However, it is similar to the development of the effective reluctance of Region 4 described by Zhu et al. in [2]. For Region 6, the reluctance network is formed by a series of parallel reluctances described by the ordinary differential equation

$$
\frac{d^{2} H_{z}}{d r^{2}}+\frac{1}{r} \frac{d H_{z}}{d r}-\alpha^{2} H_{z}=0
$$

the solution to which is given by

$$
H_{z}(r)=C_{1} I_{0}(\alpha r)+C_{2} K_{0}(\alpha r)
$$

[2]. For $r_{0}=0$, the boundary conditions $H_{z}\left(r_{1}\right)=0$ and $\left.\frac{d H_{z}(r)}{d r}\right|_{r=r_{0}}$ yield $C_{2}=0$ and $C_{1}=\frac{H_{s f}}{I_{0}\left(\alpha r_{1}\right)}[2]$, where $H_{s f}$ is the magnetic field strength at the actuator surface. However, for $r_{0}>0$ the constants become:

$$
\begin{aligned}
C_{1} & =H_{s f} \frac{1}{2} \frac{F_{M} K_{1}\left(\alpha_{1} r_{0}\right)}{I_{1}\left(\alpha_{1} r_{0}\right) K_{0}\left(\alpha_{1} r_{1}\right)+I_{0}\left(\alpha_{1} r_{1}\right) K_{1}\left(\alpha_{1} r_{0}\right)} \\
C_{2} & =\frac{1}{2} \frac{F_{M} I_{1}\left(\alpha_{1} r_{0}\right)}{I_{1}\left(\alpha_{1} r_{0}\right) K_{0}\left(\alpha_{1} r_{1}\right)+I_{0}\left(\alpha_{1} r_{1}\right) K_{1}\left(\alpha_{1} r_{0}\right)}
\end{aligned}
$$

Following the development of the effective reluctance for Region 4 described in [2]

$$
\begin{array}{r}
H_{z}(r)=H_{s f} \frac{I_{1}\left(\alpha r_{0}\right) K_{0}(\alpha r)+K_{1}\left(\alpha r_{0}\right) I_{0}(\alpha r)}{I_{0}\left(\alpha r_{1}\right) K_{1}\left(\alpha r_{0}\right)+K_{0}\left(\alpha r_{1}\right) I_{1}\left(\alpha r_{0}\right)} \\
\mu_{e f f}(r)=\mu_{r} \mu_{0} \frac{I_{1}\left(\alpha r_{0}\right) K_{0}(\alpha r)+K_{1}\left(\alpha r_{0}\right) I_{0}(\alpha r)}{I_{0}\left(\alpha r_{1}\right) K_{1}\left(\alpha r_{0}\right)+K_{0}\left(\alpha r_{1}\right) I_{1}\left(\alpha r_{0}\right)} \\
\Delta R=\frac{d_{2}}{2 \pi\left[r_{2}+\frac{i\left(r_{1}-r_{0}\right)}{n}\right] \cdot \frac{r_{1}-r_{0}}{n} \cdot \mu_{e f f}},
\end{array}
$$


where $\Delta R$ is the effective reluctance of a thin ring and $n$ is a large number of concentric rings that make up Region 6, [2]. The effective reluctance for Region 6 with $r_{0}>0$ is then

$$
R_{6}=\frac{1}{\int_{r_{0}}^{r_{1}} \frac{1}{\Delta R}}=R_{6}(s)=\frac{d_{2} \alpha}{r_{1} 2 \pi \mu_{0} \mu_{r}} \frac{I_{0}\left(\alpha r_{0}\right) K_{1}\left(\alpha r_{1}\right)+I_{1}\left(\alpha r_{1}\right) K_{0}\left(\alpha_{1} r_{0}\right)}{I_{1}\left(\alpha r_{1}\right) K_{1}\left(\alpha r_{0}\right)-I_{1}\left(\alpha r_{0}\right) K_{1}\left(\alpha r_{1}\right)}
$$

The static reluctance for Region 6 is given by

$$
R_{6}^{0}=\lim _{s \rightarrow 0} R_{6}(s)=\frac{d_{2}}{\pi \mu_{r} \mu_{0}\left(r_{1}^{2}-r_{0}^{2}\right)}
$$

which is the expected result and matches Zhu's result for $r_{0}=0$.

\subsection{Approximate Analytic Model}

The transcendental functions in the analytic model presented above are not suitable for controller design. Therefore, a simplified analytic model for $R_{1}(s)$ and $R_{6}(s)$ with rational functions, is developed in this section. Zhu et al. used Taylor series and an ad hoc approximation of $R_{i}(s)$ which is the same approach followed here with comparisons to the full model to determine the best method. A second order Taylor series expansion was used with the following form

$$
R_{i}^{T}=R_{i}^{0}+\left.\frac{1}{2} \frac{d^{2} R_{i}(v)}{d v^{2}}\right|_{v=0} \cdot v^{2}
$$

where $R_{i}^{T}$ is the Taylor approximation of $R_{i}, R_{i}^{0}$ is the static reluctance of $R_{i}$, and $v$ is the complex variable given by

$$
v= \begin{cases}\sqrt[4]{s} & i=1 \\ \sqrt{s} & i=6\end{cases}
$$

The static components of the Taylor expansion, $R_{i}^{0}$, shown above in Equations 2.12 and 2.20 , and the dynamic parts of the Taylor expansion for Regions 1 and 6 with $r_{0}>0$ are as follows:

$$
\begin{aligned}
& \left.\frac{d^{2} R_{1}}{d(\sqrt[4]{s})^{2}}\right|_{s=0}=\frac{2 r_{0}^{4} \log \frac{r_{1}}{r_{0}}+\frac{3}{2} r_{0}^{4}-2 r_{1}^{2} r_{0}^{2}+\frac{1}{2} r_{1}^{4}}{2 \pi\left(r_{0}^{2}-r_{1}^{2}\right)^{2}} \sqrt{\frac{\sigma}{\mu_{r} \mu_{0}}} \\
& \left.\frac{d^{2} R_{6}}{d(\sqrt{s})^{2}}\right|_{s=0}=\frac{d_{2} r_{0}}{2 \pi r_{1}} \frac{4 r_{0}^{2} r_{1}^{2}-r_{0}^{4}-3 r_{1}^{4}+4 r_{1}^{2} \log r_{1} / r_{0}}{\left(r_{0}^{2}-r_{1}^{2}\right)^{2}} \sigma
\end{aligned}
$$


The ad hoc approximation employed by Zhu et al. [2] uses asymptotic representations of Bessel functions such that for large values of $q$,

$$
\begin{gathered}
I_{0}(q) \approx I_{1}(q) \approx \frac{1}{\sqrt{2 \pi q}} e^{q}, \text { where }-\pi / 2<\operatorname{Arg}(q)<\pi / 2, \\
K_{0}(q) \approx K_{1}(q) \approx \sqrt{\frac{q}{2 \pi}} e^{-q}, \text { where }-\pi / 2<\operatorname{Arg}(q)<\pi / 2, \text { and } \\
\tanh (q) \approx 1 .
\end{gathered}
$$

such that for each element the ad hoc reluctance, $R_{i}^{A H}$, is written in the form

$$
R_{i}^{A H}(s)=R_{i}^{0}+c_{i}^{a} \sqrt{s}
$$

The ad hoc approximations for Regions 1 and 6 with $r_{0}>0$ are as follows

$$
\begin{aligned}
& c_{1}^{A H}(s)=\frac{g}{2 \pi \mu_{0}} \alpha_{1}(s)=\frac{\sqrt{2}}{2 \pi r_{1}} \sqrt[4]{\frac{\sigma g^{2}}{\mu_{r} \mu_{0}^{3}}} \sqrt[4]{s} \\
& c_{6}^{A H}(s)=\frac{d_{2}}{2 \pi \mu_{r} \mu_{0} r_{1}} \alpha(s)=\frac{d_{2}}{2 \pi r_{1}} \sqrt{\frac{\sigma}{\mu_{r} \mu_{0}}} \sqrt{s}
\end{aligned}
$$

The complete reluctance approximations, Taylor series and ad hoc, for Regions 1 and 6 with $r_{0}>0$ are given by

$$
\begin{aligned}
& R_{1}^{T S}(s)=\frac{l_{g}}{\pi \mu_{0}\left(r_{1}^{2}-r_{0}^{2}\right)}+\frac{2 r_{0}^{4} \log \frac{r_{1}}{r_{0}}+\frac{3}{2} r_{0}^{4}-2 r_{1}^{2} r_{0}^{2}+\frac{1}{2} r_{1}^{4}}{2 \pi\left(r_{0}^{2}-r_{1}^{2}\right)^{2}} \sqrt{\frac{\sigma}{\mu_{r} \mu_{0}}} \sqrt{s} \\
& R_{1}^{A H}(s)=\frac{l_{g}}{\pi \mu_{0}\left(r_{1}^{2}-r_{0}^{2}\right)}+\frac{\sqrt{2}}{2 \pi r_{1}} \sqrt[4]{\frac{\sigma g^{2}}{\mu_{r} \mu_{0}^{3}} \sqrt[4]{s}} \\
& R_{6}^{T S}(s)=\frac{d_{2}}{\pi \mu_{r} \mu_{0}\left(r_{1}^{2}-r_{0}^{2}\right)}+\frac{d_{2} r_{0}}{2 \pi r_{1}} \frac{4 r_{0}^{2} r_{1}^{2}-r_{0}^{4}-3 r_{1}^{4}+4 r_{1}^{2} \log r_{1} / r_{0}}{\left(r_{0}^{2}-r_{1}^{2}\right)^{2}} \sigma s \\
& R_{6}^{A H}(s)=\frac{d_{2}}{\pi \mu_{r} \mu_{0}\left(r_{1}^{2}-r_{0}^{2}\right)}+\frac{d_{2}}{2 \pi r_{1}} \sqrt{\frac{\sigma}{\mu_{r} \mu_{0}}} \sqrt{s}
\end{aligned}
$$

Ad hoc and Taylor series approximations were compared to the full analytic model by plotting magnitude and phase which are shown in Figure 2.7. For Region 1, the Taylor series matches the magnitude of the full analytic model much better than the $a d$ hoc approximation (Figure 2.7 upper left). However, the phase of the Taylor series approximation doesn't match as well as the ad hoc approximation at high frequencies, but in the low frequency range from 0 to $200 \mathrm{~Hz}$, which is the frequency range of interest because of the low bandwidth typical of thrust AMBs, the Taylor series is the better approximation (Figure 2.7 lower left). 
For Region 6, the ad hoc approximation matches the full analytic model much better in both magnitude and phase (Figure 2.7). Not surprisingly, Zhu et al. also found the Taylor series approximation to be more accurate for Region 1 and the ad hoc approximation preferable for Region 6, [3, 2]. Effective reluctances for Regions 1 through 6 are shown in Table 2.1 along with the corresponding approximations. For Regions 2 through 5, the reluctance models are the same as those found in $[3,2]$ and are shown here for completeness. Regions 1 and 6 have two effective reluctance models, one for geometries with a solid center, $r_{0}=0$, and from the extension of the model developed here, a model for geometries with a center hole, $r_{0}>0$.
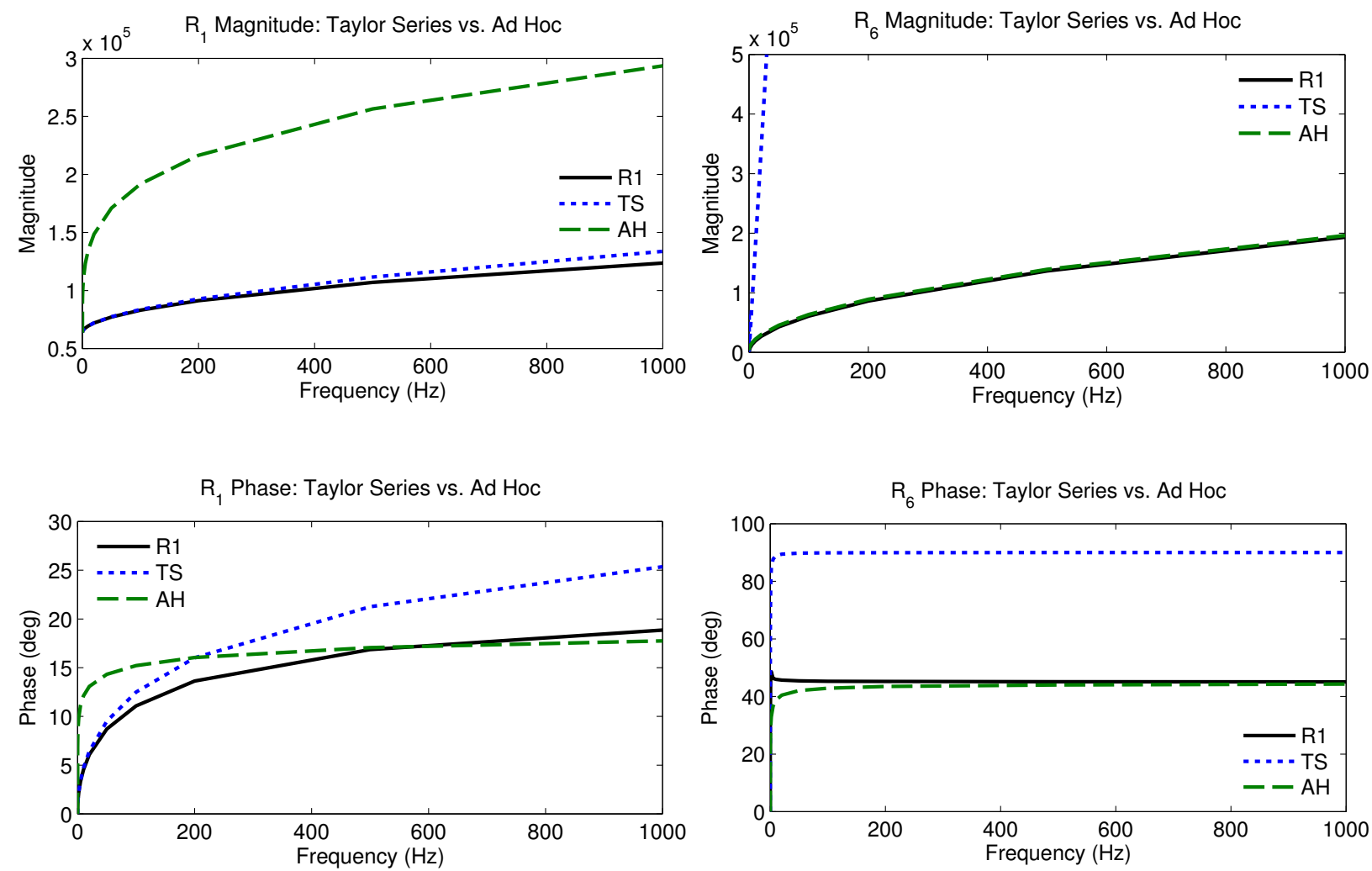

Figure 2.7: Magnitude and phase of reluctance for Regions 1 and 6 with $r_{0}>0$ approximated using Taylor series or ad hoc method.

\subsection{Current to Force Transfer Function}

With approximate reluctance models completed for geometries containing a center hole, the development of a transfer function model from perturbation force to perturbation current is undertaken in this section. The development is somewhat brief due to the fact that it follows the same process developed by Zhu et al. for axisymmetric actuators without a center hole, i.e. $r_{0}=0$, which is described in detail in [2]. From Table 2.1 
Table 2.1: Effective reluctances for Regions 1 through 6

\begin{tabular}{|c|c|c|}
\hline Region & Effective Reluctance $R_{i}(s)$ (Full Model) & Approximate Effective Reluctance $\widetilde{R}_{i}(s)$ \\
\hline $1\left(r_{0}=0\right)$ & $\frac{l_{g} \alpha_{1}}{2 \pi \mu_{0} r_{1}} \frac{I_{0}\left(\alpha_{1} r_{1}\right)}{I_{1}\left(\alpha_{1} r_{1}\right)}$ & $\frac{l_{g}}{\pi \mu_{0} r_{1}^{2}}+\frac{1}{4 \pi} \sqrt{\frac{\sigma}{\mu_{r} \mu_{0}}} \sqrt{s}$ \\
\hline $1\left(r_{0}>0\right)$ & $\frac{l_{g} \alpha_{1}}{2 \pi \mu_{0} r_{1}} \frac{I_{1}\left(\alpha_{1} r_{0}\right) K_{0}\left(\alpha_{1} r_{1}\right)+I_{0}\left(\alpha_{1} r_{1}\right) K_{1}\left(\alpha_{1} r_{0}\right)}{I_{1}\left(\alpha_{1} r_{1}\right) K_{1}\left(\alpha_{1} r_{0}\right)-I_{1}\left(\alpha_{1} r_{0}\right) K_{1}\left(\alpha_{1} r_{1}\right)}$ & $\frac{l_{g}}{\pi \mu_{0}\left(r_{1}^{2}-r_{0}^{2}\right)}+\frac{2 r_{0}^{4} \log \frac{r_{1}}{r_{0}}+\frac{3}{2} r_{0}^{4}-2 r_{1}^{2} r_{0}^{2}+\frac{1}{2} r_{1}^{4}}{2 \pi\left(r_{0}^{2}-r_{1}^{2}\right)^{2}} \sqrt{\frac{\sigma}{\mu_{r} \mu_{0}}} \sqrt{s}$ \\
\hline 2 & $\frac{\ln (r 2 / r 1)}{2 \pi \mu_{r} \mu_{0}} \frac{\alpha}{\tanh \left(\alpha d_{1}\right)}$ & $\frac{\ln \left(r_{2} / r_{1}\right)}{2 \pi \mu_{r} \mu_{0} d_{1}}+\frac{\ln \left(r_{2} / r_{1}\right)}{2 \pi} \sqrt{\frac{\sigma}{\mu_{r} \mu_{0}}} \sqrt{s}$ \\
\hline 3 & $\frac{l_{g} \alpha_{1}}{2 \pi \mu_{0} r_{2}} \frac{I_{0}\left(\alpha_{1} r_{2}\right) K_{1}\left(\alpha_{1} r_{3}\right)+K_{0}\left(\alpha_{1} r_{2}\right) I_{1}\left(\alpha_{1} r_{3}\right)}{K_{1}\left(\alpha_{1} r_{2}\right) I_{1}\left(\alpha_{1} r_{3}\right)-I_{1}\left(\alpha_{1} r_{2}\right) K_{1}\left(\alpha_{1} r_{3}\right)}$ & $\begin{array}{l}\overline{\pi \mu_{0}\left(r_{3}^{2}-r_{2}^{2}\right)} \\
+\frac{\left(2 r_{3}^{4} \log \frac{r_{3}}{r_{2}}-\frac{3}{2} r_{3}^{4}+2 r_{2}^{2} r_{3}^{2}-\frac{1}{2} r_{2}^{4}\right)}{2 \pi\left(r_{3}^{2}-r_{2}^{2}\right)^{2}} \sqrt{\frac{\sigma}{\mu_{r} \mu_{0}}} \sqrt{s}\end{array}$ \\
\hline 4 & $\frac{d_{2} \alpha}{2 \pi \mu_{r} \mu_{0} r_{2}} \frac{I_{0}\left(\alpha r_{2}\right) K_{1}\left(\alpha r_{3}\right)+K_{0}\left(\alpha r_{2}\right) I_{1}\left(\alpha r_{3}\right)}{K_{1}\left(\alpha r_{2}\right) I_{1}\left(\alpha r_{3}\right)-I_{1}\left(\alpha r_{2}\right) K_{1}\left(\alpha r_{3}\right)}$ & $\frac{d_{2}}{\pi \mu_{r} \mu_{0}\left(r_{3}^{2}-r_{2}^{2}\right)}+\frac{d_{2}}{2 \pi r_{2}} \sqrt{\frac{\sigma}{\mu_{r} \mu_{0}}} \sqrt{s}$ \\
\hline 5 & $\frac{\ln \left(r_{2} / r_{1}\right)}{2 \pi \mu_{r} \mu_{0}} \frac{\alpha}{\tanh \left(\alpha d_{3}\right)}$ & $\frac{\ln \left(r_{2} / r_{1}\right)}{2 \pi \mu_{r} \mu_{0} d_{3}}+\frac{\ln \left(r_{2} / r_{1}\right)}{2 \pi} \sqrt{\frac{\sigma}{\mu_{r} \mu_{0}}} \sqrt{s}$ \\
\hline $6\left(r_{0}=0\right)$ & $\frac{d_{2} \alpha}{2 \pi \mu_{r} \mu_{0} r_{1}} \frac{I_{0}\left(\alpha r_{1}\right)}{I_{1}\left(\alpha r_{1}\right)}$ & $\frac{d_{2}}{\pi \mu_{r} \mu_{0} r_{1}^{2}}+\frac{d_{2}}{2 \pi r_{1}} \sqrt{\frac{\sigma}{\mu_{r} \mu_{0}}} \sqrt{s}$ \\
\hline $6\left(r_{0}>0\right)$ & $\frac{d_{2} \alpha}{2 \pi \mu_{0} \mu_{r} r_{1}} \frac{I_{0}\left(\alpha r_{0}\right) K_{1}\left(\alpha r_{1}\right)+I_{1}\left(\alpha r_{1}\right) K_{0}\left(\alpha r_{0}\right)}{I_{1}\left(\alpha r_{1}\right) K_{1}\left(\alpha r_{0}\right)-I_{1}\left(\alpha r_{0}\right) K_{1}\left(\alpha r_{1}\right)}$ & $\frac{d_{2}}{\pi \mu_{r} \mu_{0}\left(r_{1}^{2}-r_{0}^{2}\right)}+\frac{d_{2}}{2 \pi r_{1}} \sqrt{\frac{\sigma}{\mu_{r} \mu_{0}}} \sqrt{s}$ \\
\hline
\end{tabular}

it can be seen that the approximate effective reluctance, denoted $\widetilde{R}_{i}$, conveniently has the same form for each region:

$$
\widetilde{R}_{i}(s)=R_{i}^{0}+c \sqrt{s}
$$

$[3,2]$. The six actuator regions are arranged in series to form a magnetic circuit with a total effective reluctance given by the sum of the effective reluctances for each region written as

$$
R=\sum_{i=1}^{6} R_{i}^{0}+c_{i} \sqrt{s} .
$$

Flux in the air gap $\left(\phi_{g}\right)$ is given by the magnetomotive force $(N I)$ divided by the reluctance $(R)$ :

$$
\phi_{g}(s)=\frac{N}{R_{e f f}(s)} I_{p}(s)=\frac{N}{\sum_{i=1}^{6} R_{i}^{0}+c_{i} \sqrt{s}} I_{p}(s)
$$

The perturbation force $F_{p}$ is then determined using the Maxwell stress tensor $[3,1,10]$.

$$
f(t)=\frac{1}{2 \mu_{0}} \oint_{S} \mathbf{B}^{2}(t, r) \mathrm{d} S,
$$


such that for a constant bias flux and time-varying perturbation flux the total flux density is $\mathbf{B}=\mathbf{B}_{b}+\mathbf{B}_{p}$, and the total force becomes

$$
f(t)=\frac{1}{2 \mu_{0}} \oint_{S}\left[\mathbf{B}_{b}^{2}+2 \mathbf{B}_{b} \mathbf{B}_{p}(t, r)+\mathbf{B}_{p}^{2}(t, r)\right] \mathrm{d} S .
$$

The perturbation force for a given perturbation current may be derived via the representation of the total perturbation flux. The rational behind this is that for a small perturbation current $\mathbf{B}_{p}^{2}(t, r) \approx 0$ and therefore the perturbation force is a function of the sum of the bias flux density and the perturbation flux density over the inner pole surface area $\left(A_{1}\right)$ and the outer pole surface area $\left(A_{2}\right)$ :

$$
\begin{aligned}
F_{p}(s) & =\frac{1}{\mu_{0}}\left[\int_{r_{0}}^{r_{1}} \mathbf{B}_{b} \mathbf{B}_{p}(s, r) \cdot 2 \pi r \mathrm{~d} r+\int_{r_{2}}^{r_{3}} \mathbf{B}_{b} \mathbf{B}_{p}(s, r) \cdot 2 \pi r \mathrm{~d} r\right] \\
& =\frac{\phi_{b}}{\mu_{0}}\left(\frac{1}{A_{1}}+\frac{1}{A_{2}}\right) \phi_{p}(s) \\
=\frac{\phi_{b}}{\mu_{0}} & \left(\frac{1}{A_{1}}+\frac{1}{A_{2}}\right)\left[\frac{N}{\sum_{i=1}^{6} R_{i}^{0}+c_{i} \sqrt{s}}\right] i_{p}(s),
\end{aligned}
$$

where the total bias and perturbation flux in the air gap $\left(\phi_{b}\right.$ and $\left.\phi_{p}\right)$, rather than flux densities at each position, are sufficient to determine the perturbation force.

\subsection{Verification of the Analytic Model}

To verify the accuracy of the analytic model for magnetic actuator geometries with a center hole, several test cases were chosen to analyze and compare with FEA results. The first test case is similar to an existing thrust bearing on a compressor surge test rig developed at the University of Virginia [24, 25]. Several other test cases are derivations of the first with geometric and material properties summarized in Table 2.2. Most of these parameters are defined in Figure 2.1 with the exception of $r_{0}$ which has been introduced as the radius of the center hole, or inner radius of the actuator. Zhu et al. performed a similar verification study and included various material properties which agreed well with predictions made via FEA [3, 2]. Since modifications to the model were to extend the type of geometry covered, verification here will focus on geometric variations rather than variations of material properties. Finite element analysis was performed using Finite Element Method Magnetics (FEMM) [23] over a range of current excitation frequencies from static to $400 \mathrm{~Hz}$. Flux magnitude and phase were plotted to determine the minimal mesh resolutions required ( Figure 2.8).

Flux magnitudes are similar for each of the mesh resolutions tested in FEMM (Figure 2.8). However, for the lowest resolution mesh, the phase diverges from that of the finer meshes for frequencies above $100 \mathrm{~Hz}$ 
(Figure 2.8). At higher frequencies the eddy-current induced skin effect restricts the flux path to the surface region adjacent to the coil (Figure 2.4). With the flux concentrated in fewer elements near the surface and a steep flux gradient at the edge of this region a finer mesh resolution is required in order to obtain accurate results. The highest mesh resolution (6 million nodes for Case 1) was used for all subsequent finite element analysis shown in this thesis that was performed using FEMM.

Table 2.2: Thrust bearing parameters

\begin{tabular}{lcccccccccccc} 
Case & $r_{0}(\mathrm{~mm})$ & $r_{1}(\mathrm{~mm})$ & $r_{2}(\mathrm{~mm})$ & $r_{3}(\mathrm{~mm})$ & $d_{1}(\mathrm{~mm})$ & $d_{2}(\mathrm{~mm})$ & $d_{3}(\mathrm{~mm})$ & $l_{g}(\mathrm{~mm})$ & $\sigma(\mathrm{MS} / \mathrm{m})$ & $\mu_{r}$ & $N$ (num. turns) \\
\hline 1 & 51.7 & 68.3 & 90.9 & 101.3 & 16.5 & 26.3 & 15.2 & 0.5 & $2 \times 10^{6}$ & 1000 & 166 \\
2 & 51.7 & 68.3 & 90.9 & 101.3 & 16.5 & 26.3 & 15.2 & 1.0 & $2 \times 10^{6}$ & 1000 & 166 \\
3 & 51.7 & 68.3 & 113.5 & 122 & 16.5 & 26.3 & 15.2 & 0.5 & $2 \times 10^{6}$ & 1000 & 332 \\
4 & 51.7 & 68.3 & 90.9 & 101.3 & 16.5 & 52.6 & 15.2 & 0.5 & $2 \times 10^{6}$ & 1000 & 332 \\
5 & 103.4 & 112.6 & 135.2 & 142.4 & 16.5 & 26.3 & 15.2 & 0.5 & $2 \times 10^{6}$ & 1000 & 166 \\
6 & 13.0 & 46.5 & 69.1 & 82.3 & 16.5 & 26.3 & 15.2 & 0.5 & $2 \times 10^{6}$ & 1000 & 166 \\
\hline
\end{tabular}

Magnitude: 2-D mesh analysis in FEMM

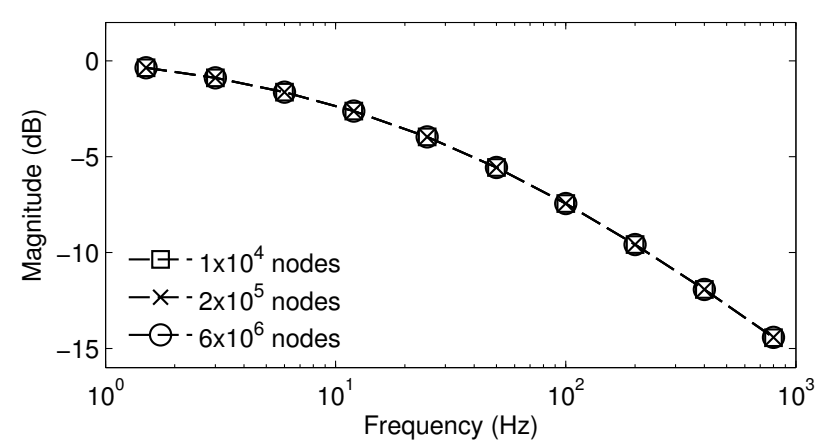

Phase: 2-D mesh analysis in FEMM

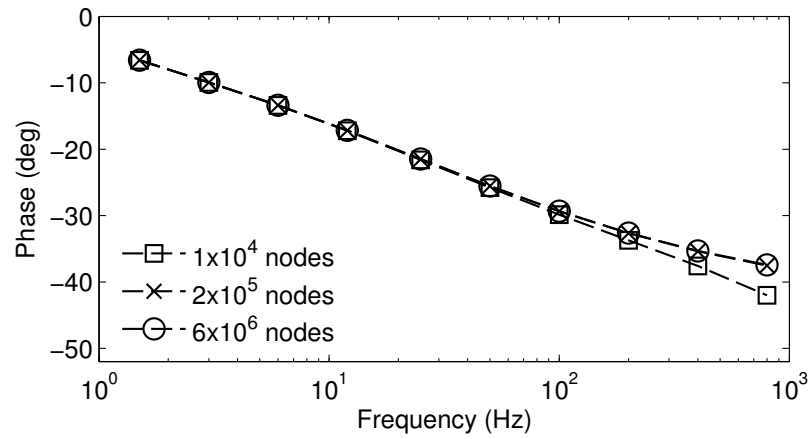

Figure 2.8: 2-D finite element modeling shows the flux magnitude and phase for three mesh resolutions.

Flux magnitude and phase for several geometries were predicted using the full and approximate analytic models and are compared to predictions from FEA models that either include or exclude flux leakage and fringing (Figures 2.9- 2.14). Flux magnitudes for each model were normalized to the static results of the FEA model, which allows leakage and fringing, as shown by

$$
M_{n o r m}=20 \log \left|\frac{G_{i}(j \omega)}{G_{L F}(0)}\right| \text {, }
$$

where $M_{n o r m}$ is the normalized flux magnitude in $d B, G_{i}(s)$ represents the analytic or FEA model being normalized, and $G_{L F}(0)$ represents the FEA model with leakage and fringing with a static perturbation current. The magnetic circuit model used to develop the analytic model does not account for flux leakage or fringing and is therefore expected to match the results of FEA that do not allow leakage or fringing which is consistent with the results presented here. Additive error (AE), multiplicative error (ME), and phase error 
(PE), commonly used in robust control design [2], were determined for the analytic models relative to the FEA models. Results are shown in Figures 2.9 through 2.14.

$$
\begin{aligned}
A E(s) & =\frac{\left|G_{A}(j \omega)-G_{F E A}(j \omega)\right|}{G_{F E A}(0)} \\
M E(s) & =\frac{\left|G_{A}(j \omega)-G_{F E A}(j \omega)\right|}{\left|G_{F E A}(j \omega)\right|} \\
P E(s) & =\angle G_{i}(j \omega)-\angle G_{F E A}(j \omega)
\end{aligned}
$$

where $G_{A}(s)$ represents an analytic model, full or approximate, and $G_{F E A}(s)$ represents a FEA model, either with or without leakage and fringing. Comparison of the analytic models with the FEA model without leakage and fringing effects, offers a more fundamental comparison, while comparison to the FEA model including leakage and fringing is a more practical assessment. The full analytic model agrees very closely with the FEA model that does not include leakage or fringing. In most cases the analytic models also agree well with FEA models that do allow leakage and fringing. However, for a large air gap or a wide coil groove, the analytic models tend to underestimate the total flux magnitude, more so at the lower frequencies, with the largest errors seen in Case 3 (Figure 2.11), where the maximum AE and ME are 0.15 and $16 \%$, respectively. The geometry of test Case 3 has a coil groove twice as wide as the standard geometry, Case 1, which is an extreme not likely to appear in an actual magnetic bearing. Even with this large coil groove, the analytic models agree well with the FEA model neglecting leakage and fringing, which strongly supports their validity. Leakage and fringing do not have much effect on phase, and in all cases, the full and approximate analytic models agree very closely with FEA models on phase with the largest error in phase (4 deg) appearing in test Cases 4 and 5 (Figures 2.12 and 2.13). The approximate analytic model also agrees well with FEA results with the exception that it estimates slightly lower force magnitude in the low to meddium frequency range. 
Flux Magnitude

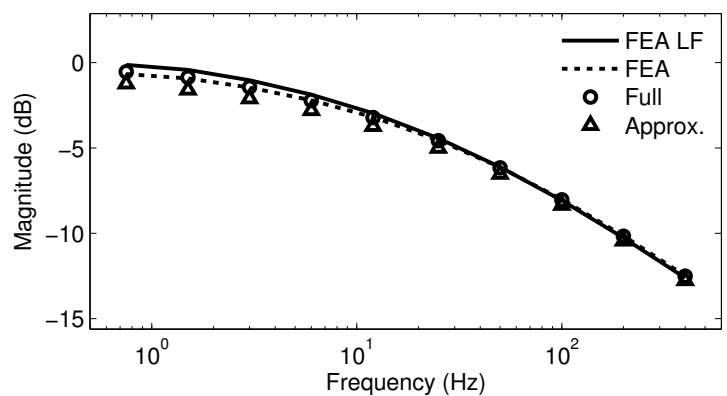

Magnitude Error, FEA without FL

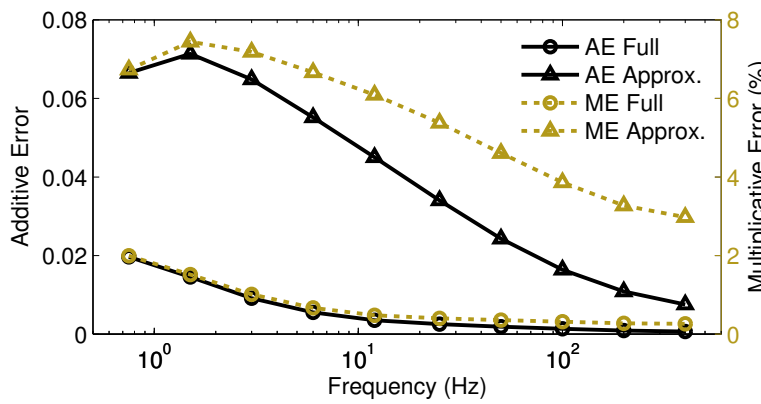

Magnitude Error, FEA with FL

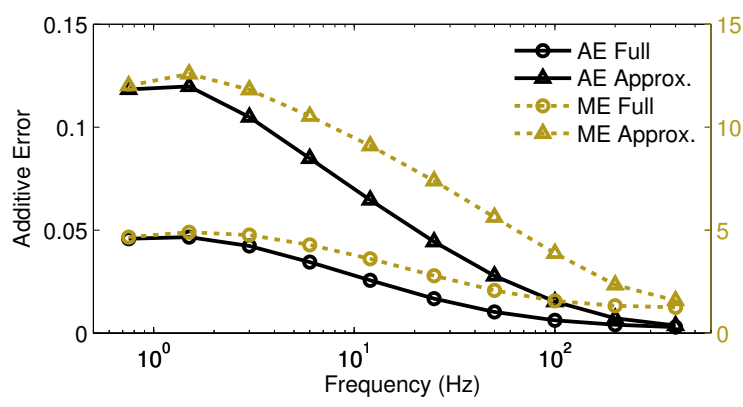

Flux Phase

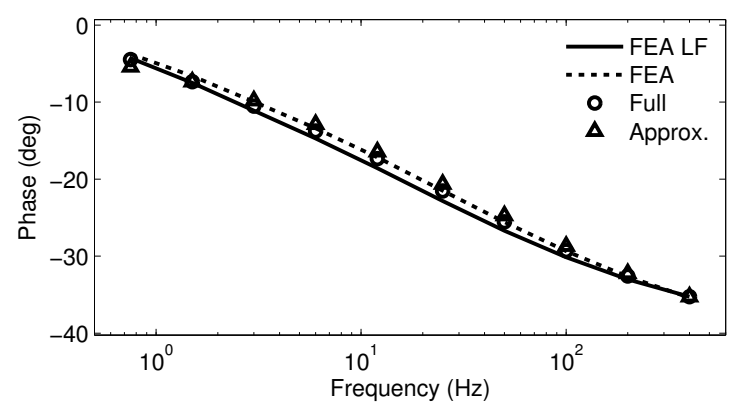

Phase Error, FEA without FL

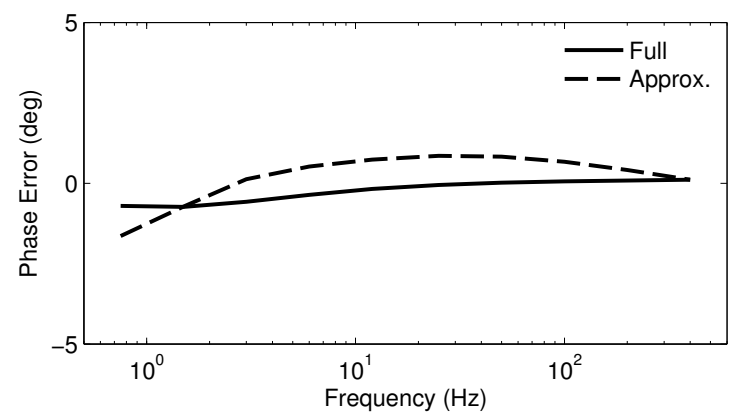

Phase Error, FEA with FL

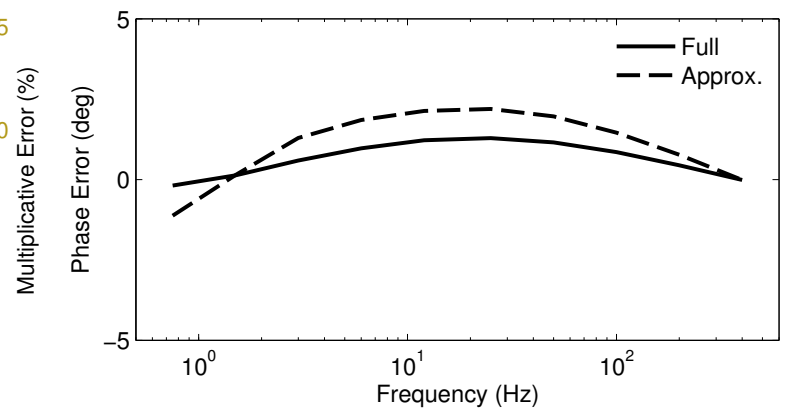

Figure 2.9: Case 1 (compressor thrust AMB): Flux magnitude and phase predicted by FEA model without flux leakage or fringing (FEA), with leakage and fringing (FEA LF), the full analytic model (Full), or the simplified analytic model (Approx.). Additive, Multiplicative, and Phase Error are also shown for the full and approximate analytic models relative to the FEA models with and without leakage and fringing. 
Flux Magnitude

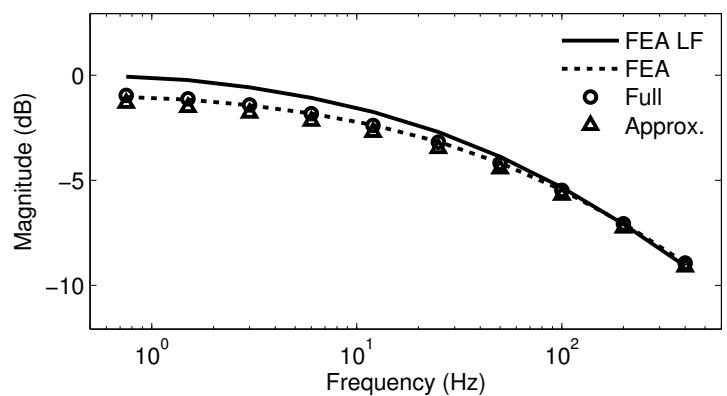

Magnitude Error, FEA without FL

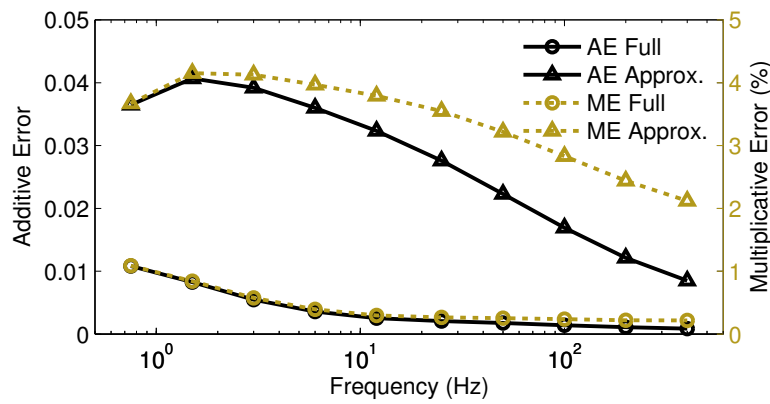

Magnitude Error, FEA with FL

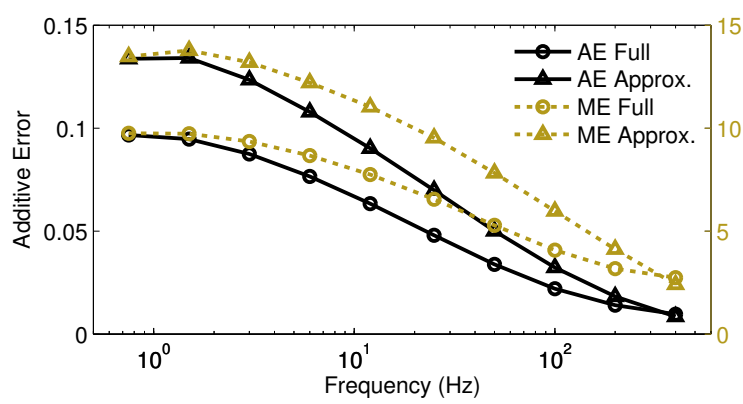

Flux Phase

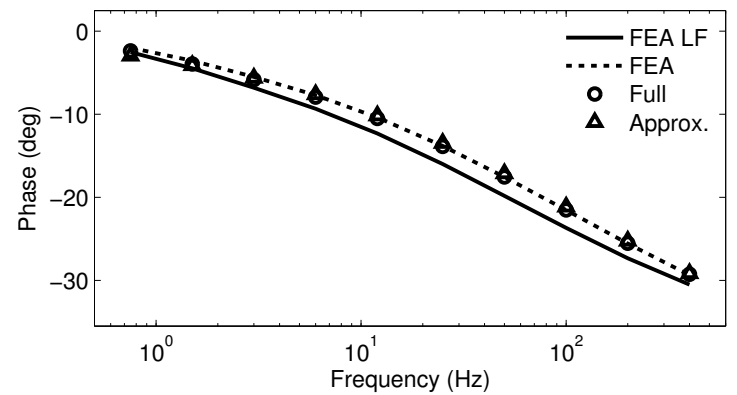

Phase Error, FEA without FL

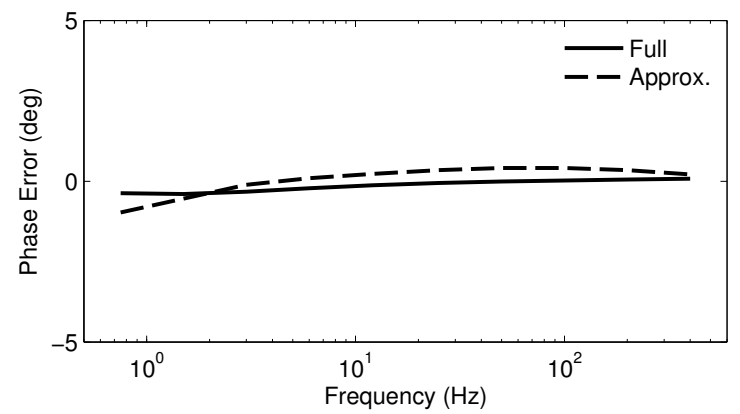

Phase Error, FEA with FL

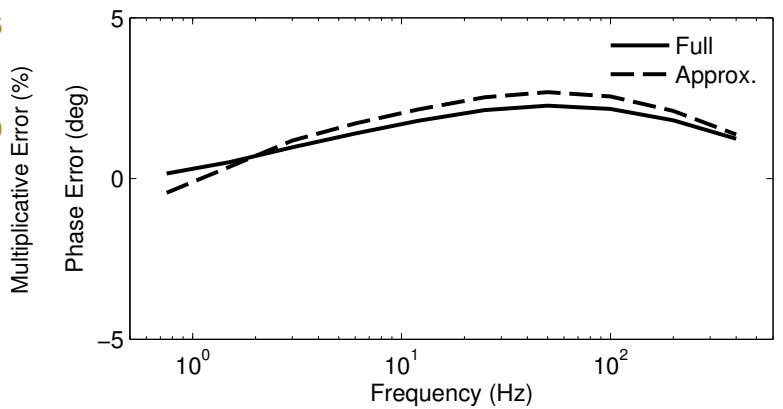

Figure 2.10: Case 2 (1mm air gap): Flux magnitude and phase predicted by FEA model without flux leakage or fringing (FEA), with leakage and fringing (FEA LF), the full analytic model (Full), or the simplified analytic model (Approx.). Additive, Multiplicative, and Phase Error are also shown for the full and approximate analytic models relative to the FEA models with and without leakage and fringing. 
Flux Magnitude

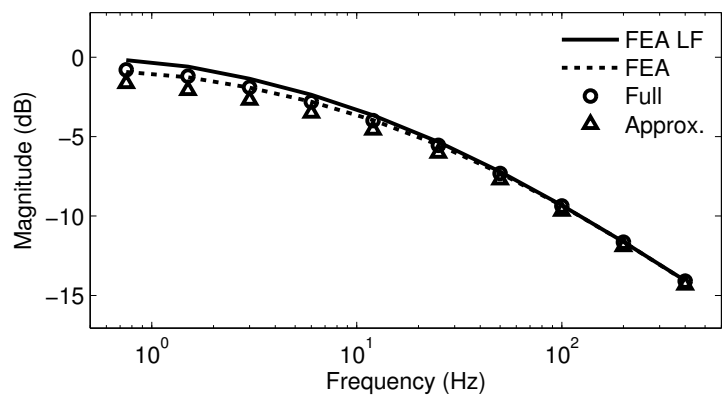

Magnitude Error, FEA without FL

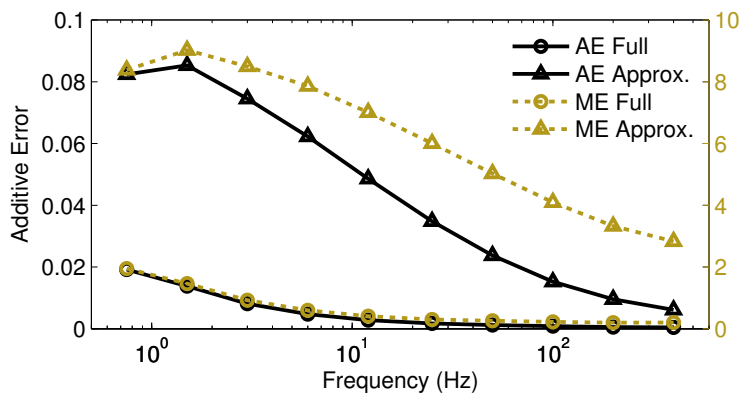

Magnitude Error, FEA with FL

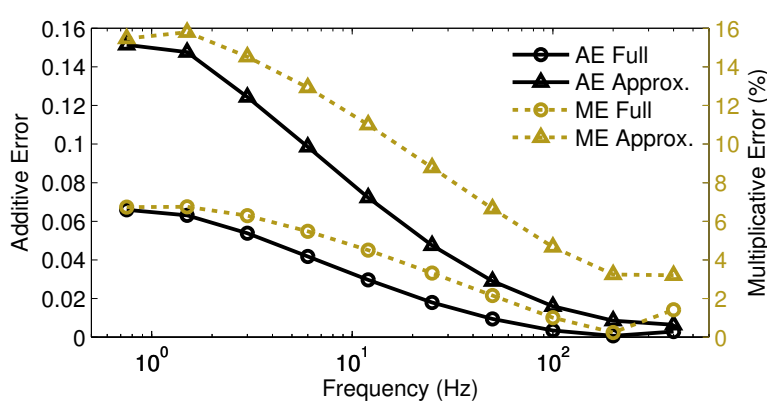

Flux Phase

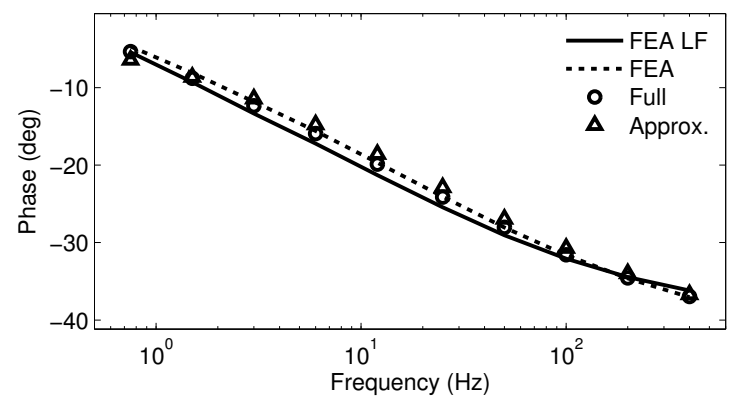

Phase Error, FEA without FL

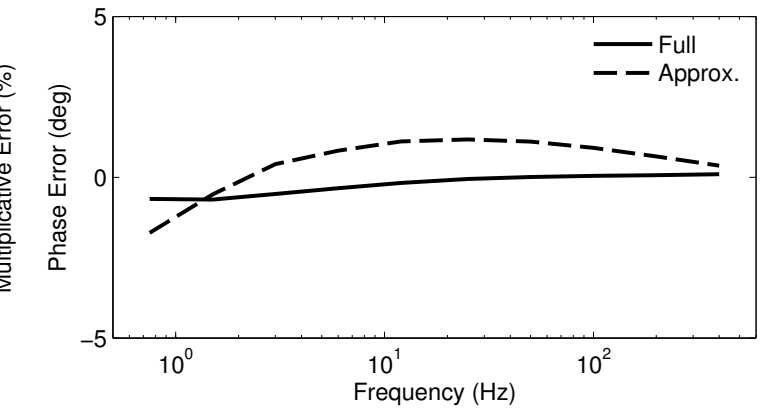

Phase Error, FEA with FL

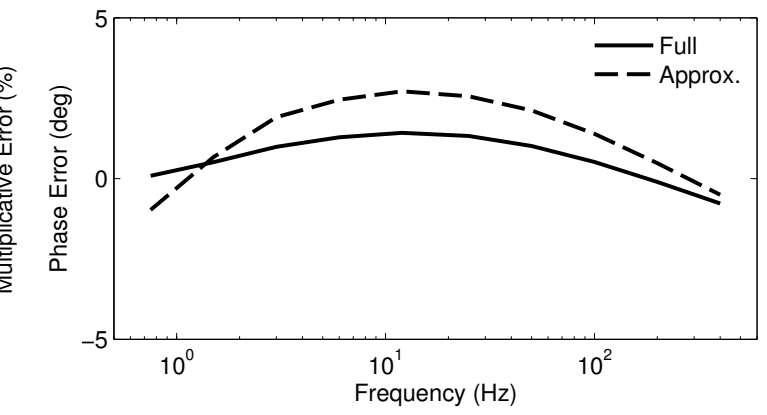

Figure 2.11: Case 3 (wide coil groove): Flux magnitude and phase predicted by FEA model without flux leakage or fringing (FEA), with leakage and fringing (FEA LF), the full analytic model (Full), or the simplified analytic model (Approx.). Additive, Multiplicative, and Phase Error are also shown for the full and approximate analytic models relative to the FEA models with and without leakage and fringing. 
Flux Magnitude

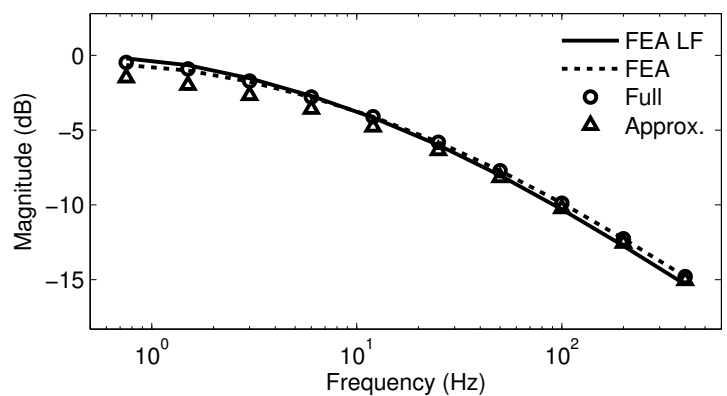

Magnitude Error, FEA without FL

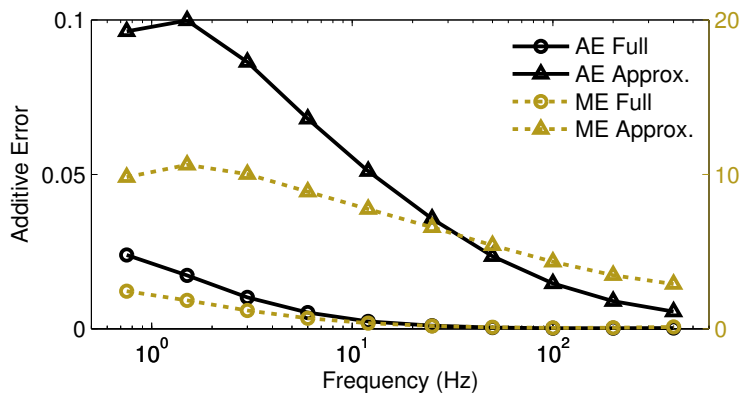

Magnitude Error, FEA with FL

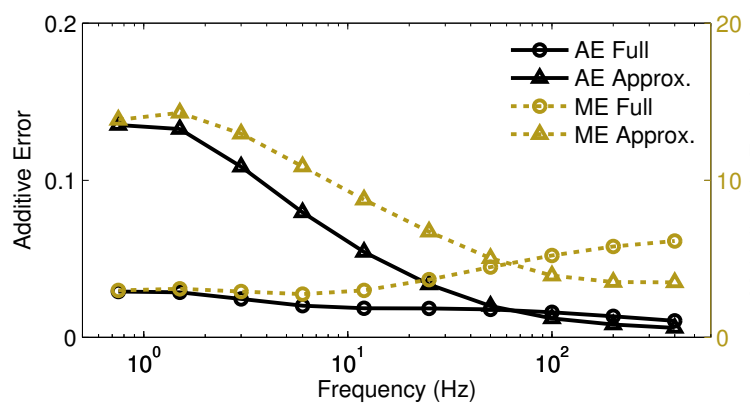

Flux Phase

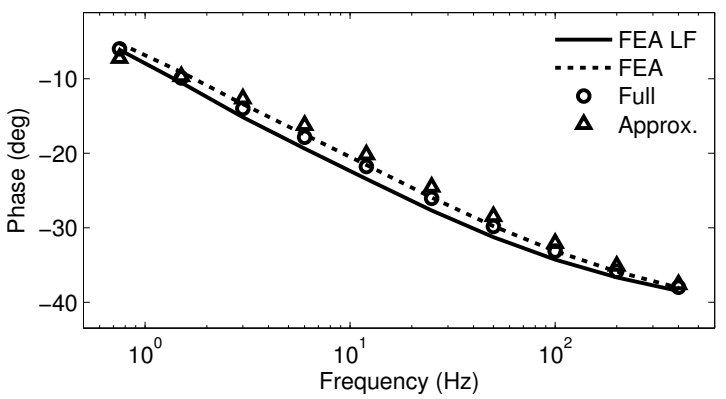

Phase Error, FEA without FL

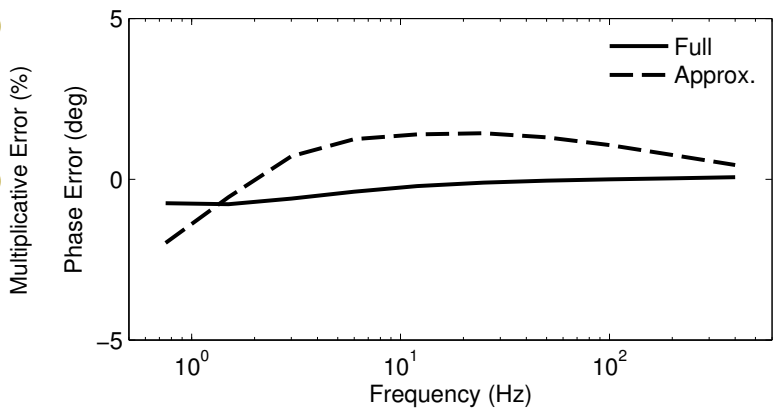

Phase Error, FEA with FL

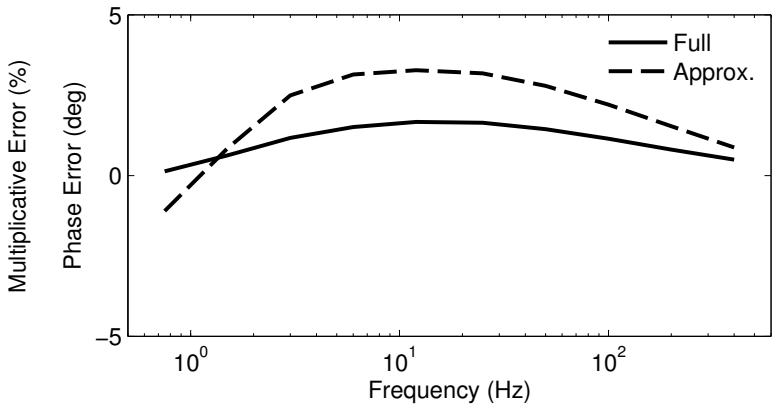

Figure 2.12: Case 4 (deep coil groove): Flux magnitude and phase predicted by FEA model without flux leakage or fringing (FEA), with leakage and fringing (FEA LF), the full analytic model (Full), or the simplified analytic model (Approx.). Additive, Multiplicative, and Phase Error are also shown for the full and approximate analytic models relative to the FEA models with and without leakage and fringing. 
Flux Magnitude

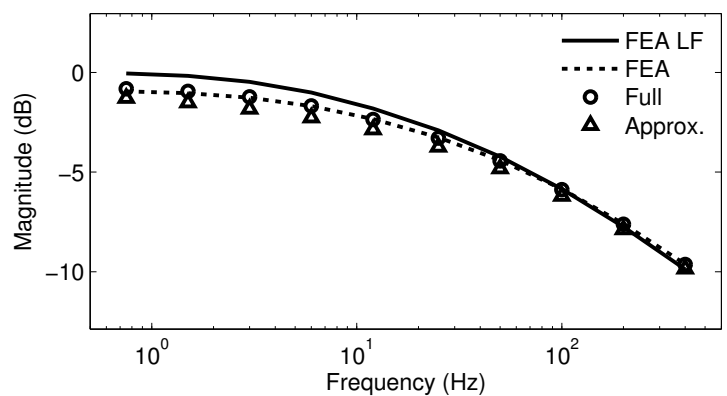

Magnitude Error, FEA without FL

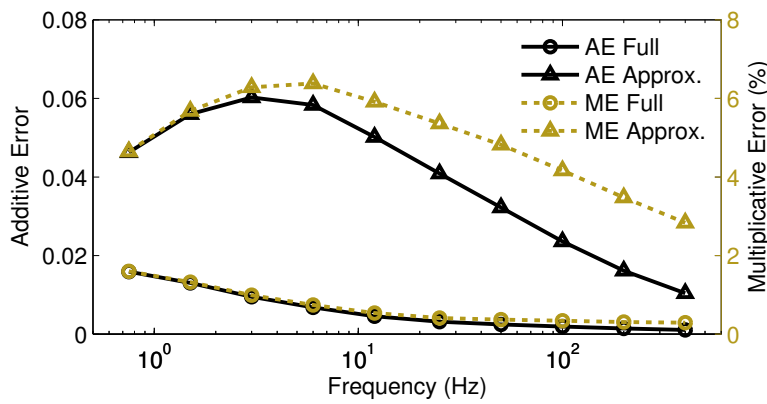

Magnitude Error, FEA with FL

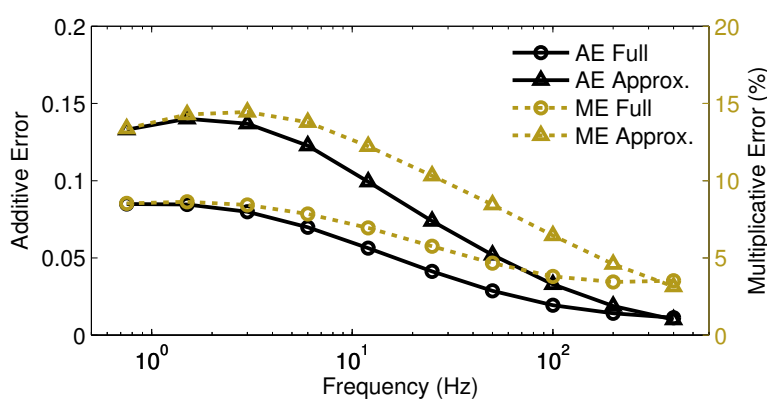

Flux Phase

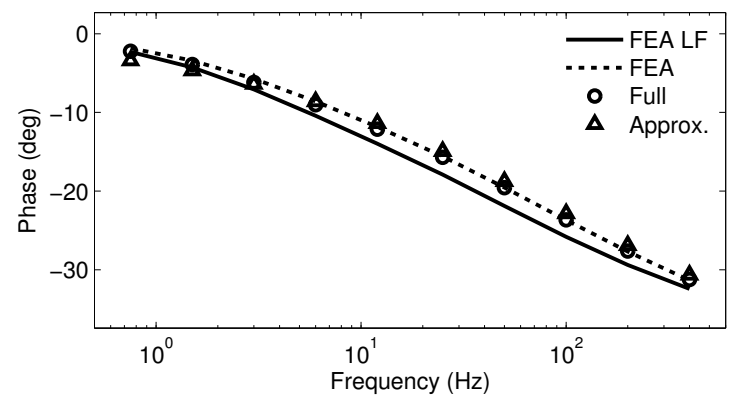

Phase Error, FEA without FL

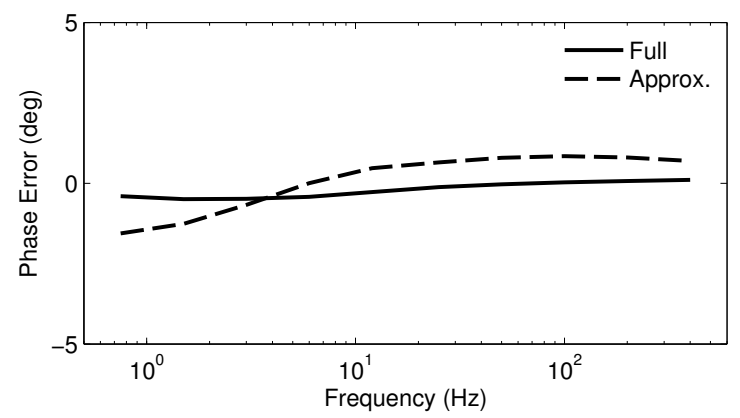

Phase Error, FEA with FL

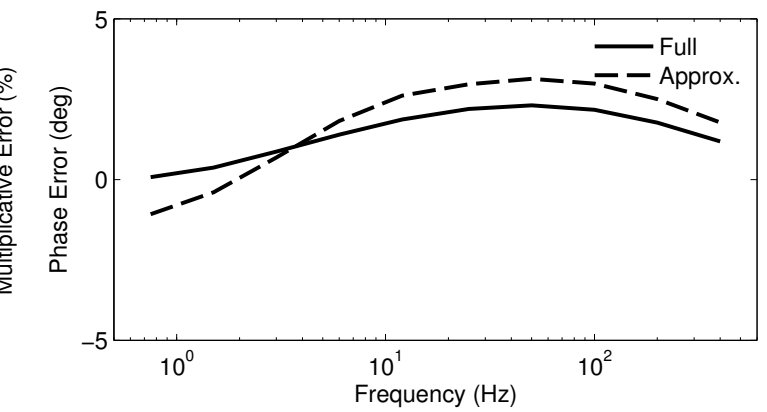

Figure 2.13: Case 5 (large inner radius): Flux magnitude and phase predicted by FEA model without flux leakage or fringing (FEA), with leakage and fringing (FEA LF), the full analytic model (Full), or the simplified analytic model (Approx.). Additive, Multiplicative, and Phase Error are also shown for the full and approximate analytic models relative to the FEA models with and without leakage and fringing. 
Flux Magnitude

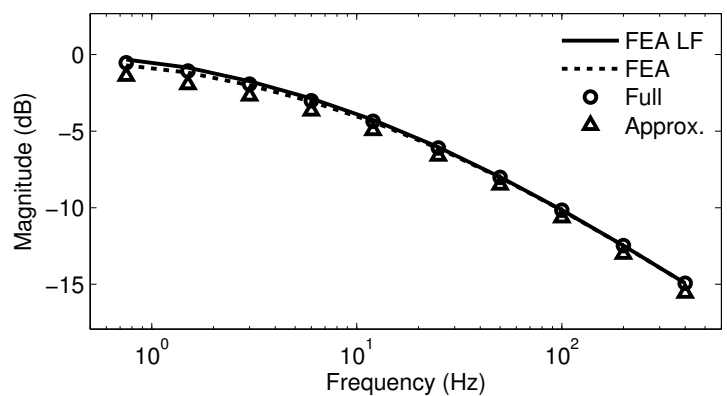

Magnitude Error, FEA without FL

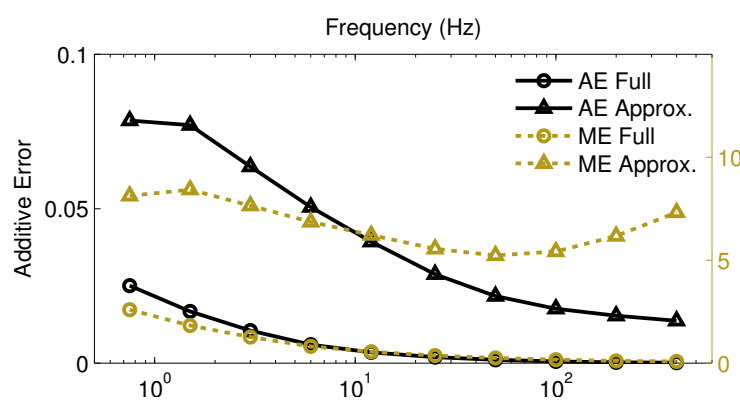

Magnitude Error, FEA with FL

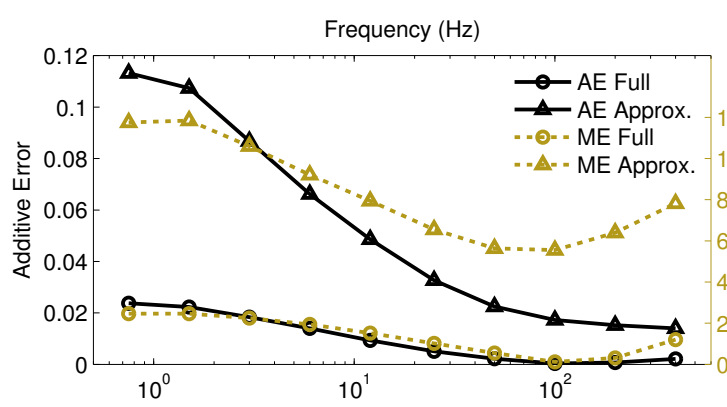

Flux Phase

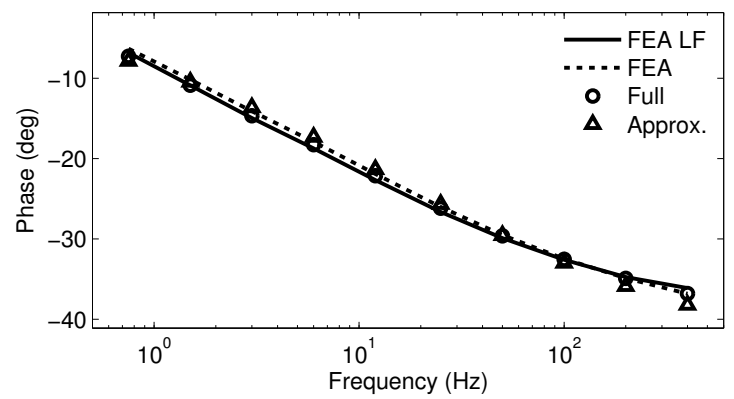

Phase Error, FEA without FL

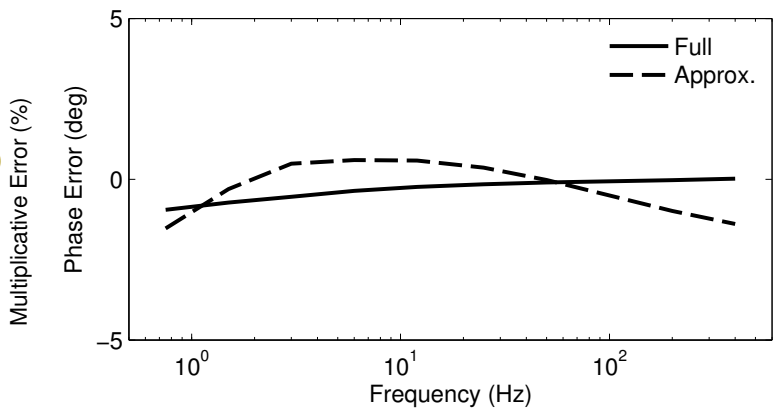

Phase Error, FEA with FL

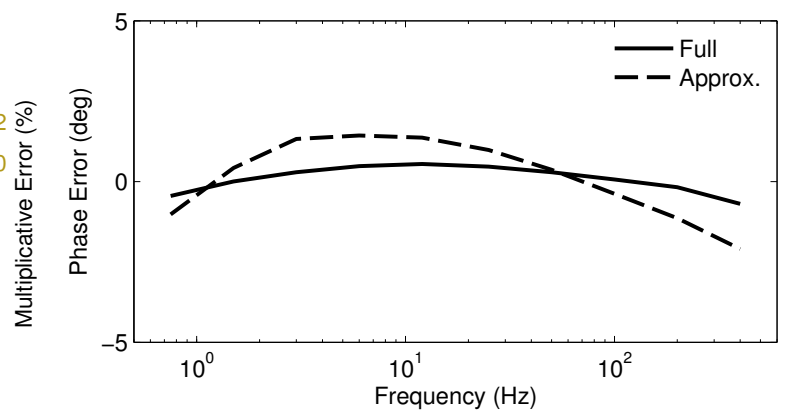

Figure 2.14: Case 6 (small inner radius): Flux magnitude and phase predicted by FEA model without flux leakage or fringing (FEA), with leakage and fringing (FEA LF), the full analytic model (Full), or the simplified analytic model (Approx.). Additive, Multiplicative, and Phase Error are also shown for the full and approximate analytic models relative to the FEA models with and without leakage and fringing. 


\section{Chapter 3}

\section{Analytic Model for Segmented Thrust}

\section{AMBs}

In this chapter an analytic model for solid segmented thrust AMBs with an arbitrary number of stator cuts is developed by adapting the analytic models for C-type non-laminated magnetic actuators developed by Zhu et al. [3, 1, 2]. The analytic model is verified by FEA and together these results show that stator segmentation improves dynamic performance of thrust AMBs. For segmented thrust bearings the analytic model is especially critical because of the time and computational resources required for FEA of segmented geometries. Segmentation disrupts the axial symmetry of the geometry which means FEA must be done in 3-D which is much more computationally intensive than 2-D FEA. For example, some of the analysis presented below, performed in ANSYS on the University of Virginia's high performance computing cluster, required seven days to solve for a single perturbation frequency.

An axisymmetric thrust bearing cut like a pie has stator segments that resemble individual C-type actuators that are curved and fit together. Figure 3.1 shows a depiction of a single segment of a thrust AMB with a cut stator. It can be seen that the geometry of the cut stator resembles that of a C-type actuator with some exceptions: the stator is curved, the pole widths and lengths are different for the inner and outer poles, and the flotor extends beyond the edges of the stator. All of these differences will be address in the segmented stator model derivation presented in the following sections. 


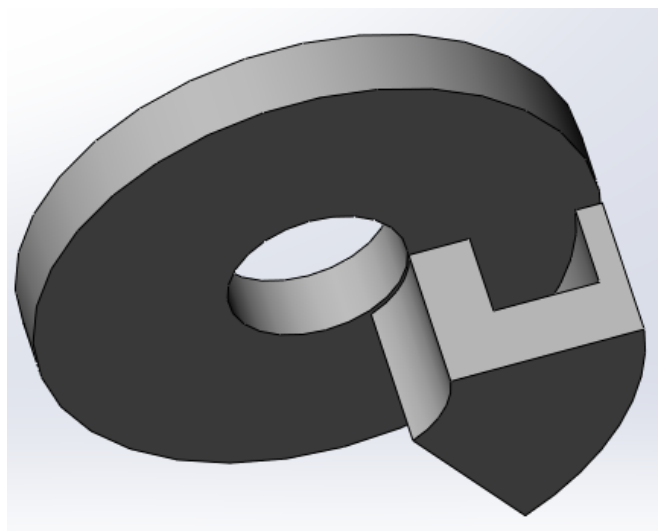

Figure 3.1: Depiction of a single segment of a cut thrust AMB.

\subsection{Analysis of Extending Flotor}

To demonstrate the aplicability of a C-type analytic model to a segmented geometry will be accurate, FEA was first performed to show that a large flotor extending beyond the edge of the stator does not significantly change the behavior of a magnetic actuator in comparison to a flotor without overhanging material. 2-D FEA using FEMM was carried out to compare the total flux over a range of frequencies for an axisymmetric geometry with a normal size flotor and an extended flotor. The geometry of Case 1 from Chapter 2 has been modified so that the outer radius of the flotor $\left(r_{3}\right)$ is increased by $50 \%$. Flux contours for the static case (Figure 3.2) show that the flux path does extend into the overhanging portion of the flotor. However, for a static perturbation the reluctance in the iron is low and the total reluctance is determined primarily by the width of the air gap. As the frequency increases, the flux path looks more like that of the standard sized flotor shown for Case 1 in Figure 2.4. At higher frequencies, the skin effect restricts the flux to the region close to the flotor surface, away from the overhung portion of the flotor. For these reasons the effective reluctance, and resulting flux, are not significantly different for a flotor extending beyond the edge of the stator, compared to a flotor matched to the stator. This can be seen clearly in Figure 3.4, which shows normalized flux magnitude and phase for Case 1 with a typical flotor compared to an overhung flotor. These results demonstrate that flotor material beyond the footprint of the stator has little effect on the frequency response. Therefore, stator segments may be analyzed independently of the rest of the actuator, allowing the results for C-type geometries to be adapted to segmented thrust bearings.

\subsection{Zhu's Model for C-type Actuators}

In developing an analytic model for thrust AMBs with segmented stators, the starting point was a model for C-type actuators developed by Zhu et al., [1,2], which will be summarized here. Development of the model 


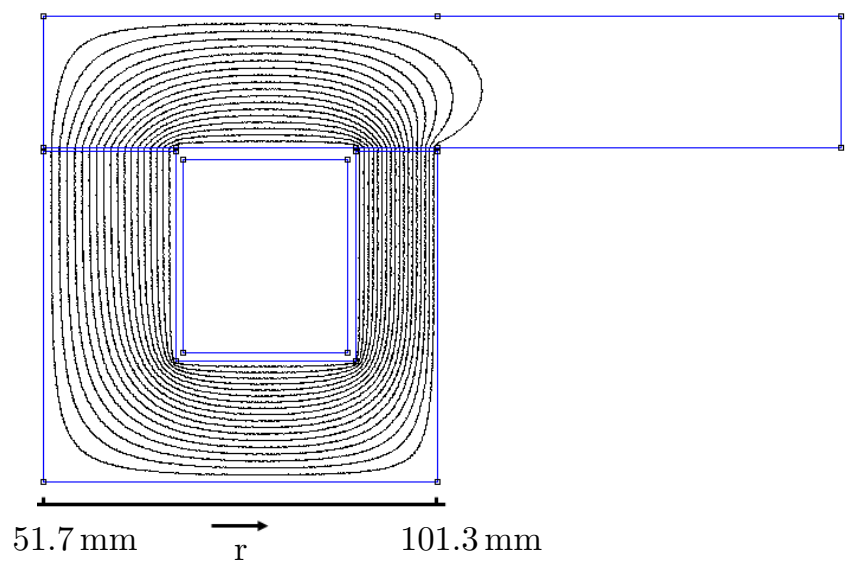

Figure 3.2: Flux contours with oversized flotor, static perturbation current.
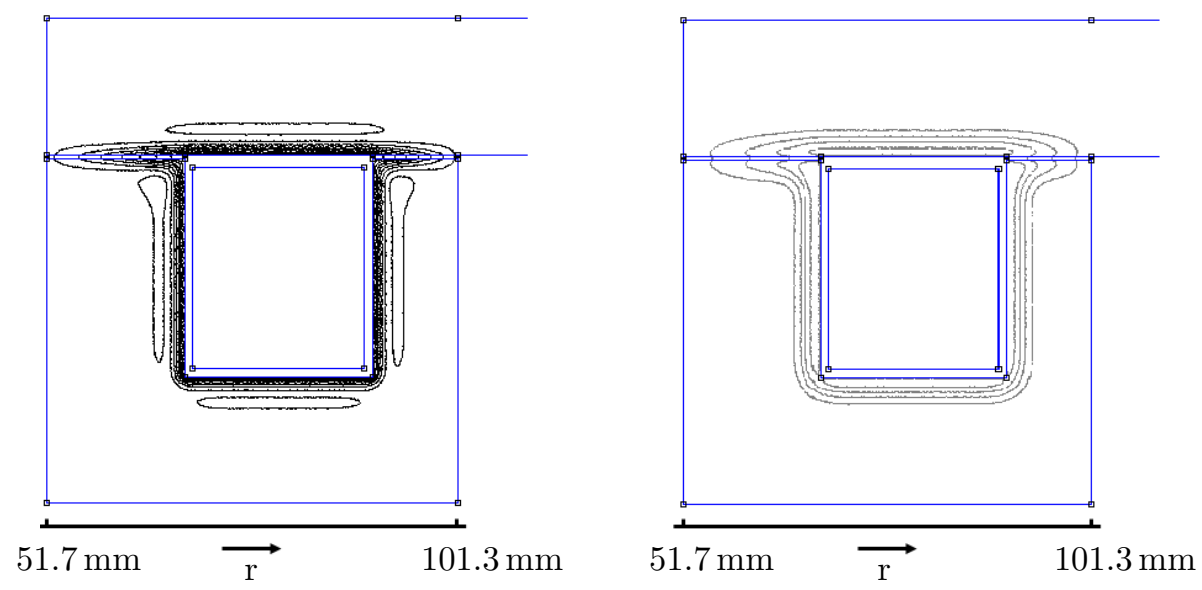

Figure 3.3: Flux contours real (left) and imaginary (right) with oversized flotor, $50 \mathrm{~Hz}$ perturbation current.

for C-type actuators follows a similar path to that of axisymmetric geometries discussed in Chapter 2 and is based on the same ideas of skin effect and effective reluctance. Just as for the axisymmetric case, the C-type geometry is divided into discrete regions and an effective reluctance is determined for each region. For the C-type geometry, Zhu's model uses four regions: one region for each air gap, one for the flotor, and one for the stator. The model could be considered as having only two regions because the reluctance models for each air gap are identical, as are the reluctance models for the stator and flotor.

Zhu's effective reluctance for the air gap regions is given by

$$
R_{g}=\frac{l_{g}}{\mu_{0}} \frac{1}{\frac{4 a \tanh \left(\alpha_{1} b\right)}{\alpha_{1}} \sum_{n=1}^{\infty}\left(\frac{4 \sqrt{2} \alpha_{1} \sqrt{b}}{(2 n-1) \pi \beta_{1 n}^{3 / 2}}\right)^{2} \tanh \left(\beta_{1 n} a\right)},
$$



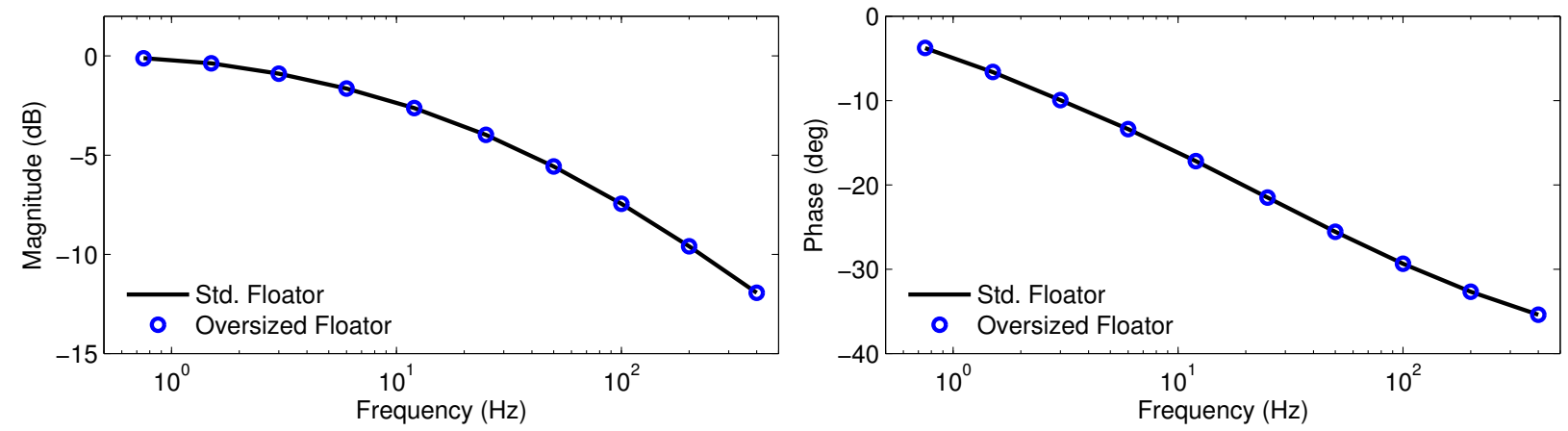

Figure 3.4: Total flux, magnitude and phase, for standard (Chapter 2 Case 1, and overhung flotor analyzed by 2 -D FEA.

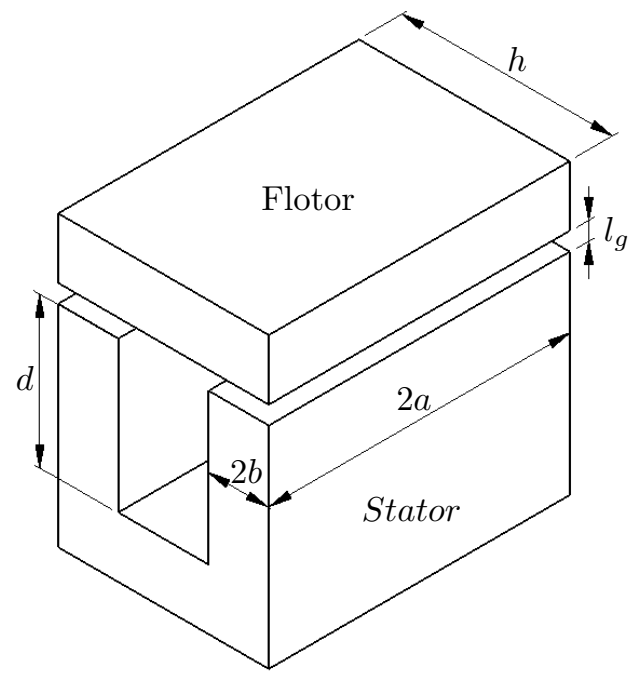

Figure 3.5: C-type actuator geometry with parameters.

where $\alpha_{1}=\sqrt{\frac{2 \alpha}{\mu_{r} l_{g}}}$ and $\alpha=\sqrt{s \sigma \mu_{r} \mu_{0}} ; a, b, d, h$, and $l_{g}$ are geometric parameters defined in Figure 3.5; and $\beta_{1 n}^{2}$ is given by

$$
\beta_{1 n}^{2}=\alpha_{1}^{2}+\frac{(2 n-1)^{2} \pi^{2}}{4 b^{2}}
$$

[2]. By taking the limit as s approaches zero, Zhu determined the static reluctance of an air gap Region to be

$$
R_{g}^{0}=\lim _{s \rightarrow 0}=\frac{l_{g}}{\mu_{0} 4 a b}
$$

[2]. $R_{g}$ is written more conveniently in terms of the static reluctance as

$$
R_{g}=R_{g}^{0} \frac{1}{\frac{4 a \tanh \left(\alpha_{1} b\right)}{\alpha_{1} b} \sum_{n=1}^{\infty} \frac{8}{(2 n-1)^{2} \pi^{2}} \frac{\alpha_{1}^{2}}{\beta_{1 n}^{2}} \frac{\tanh \left(\beta_{1 n} a\right)}{\beta_{1 n} a}}
$$


[2]. Zhu also developed a rational approximation of the effective reluctance for the air gap region given by

$$
\widetilde{R}=R_{g}^{0}+\left[\frac{b}{6 a}-\frac{32 b^{2}}{\pi^{5} a^{2}} \tanh \left(\frac{\pi a}{2 b}\right)\right] \sqrt{\frac{\sigma}{\mu_{r} \mu_{0}}} \sqrt{s}
$$

The effective reluctance of the flotor and stator regions is given by

$$
R_{i}=\frac{l_{i}}{\mu_{r} \mu_{0}} \frac{1}{\frac{4 a \tanh (\alpha b)}{\alpha} \sum_{n=1}^{\infty}\left(\frac{4 \sqrt{2} \alpha_{1} \sqrt{b}}{(2 n-1) \pi \beta_{2 n}^{3 / 2}}\right)^{2} \tanh \left(\beta_{2 n} a\right)}
$$

where $l_{i}$ is the length of the iron region, $l_{i}=h$ for the flotor and $l_{i}=h+2 c$ for the stator, and $\beta_{2 n}^{2}$ is given by

$$
\beta_{2 n}^{2}=\alpha^{2}+\frac{(2 n-1)^{2} \pi^{2}}{4 b^{2}}
$$

[2]. The static reluctance of an iron region, stator or flotor, is

$$
R_{i}^{0}=\lim _{s \rightarrow 0}=\frac{l_{i}}{\mu_{0} 4 a b}
$$

[2]. The effective reluctance of the flotor and stator regions is also written more conveniently in terms of the static reluctance, as

$$
R_{i}=R_{i}^{0} \frac{1}{\frac{4 a \tanh (\alpha b)}{\alpha} \sum_{n=1}^{\infty} \frac{8}{(2 n-1)^{2} \pi^{2}} \frac{\alpha_{1}^{2}}{\beta_{1 n}^{2}} \frac{\tanh \left(\beta_{1 n} a\right)}{\beta_{1 n} a}},
$$

and has a rational approximation given by

$$
\widetilde{R}_{i}=R_{i}^{0}+\frac{l_{i}}{4(a+b)} \sqrt{\frac{\sigma}{\mu_{r} \mu_{0}}} \sqrt{s}
$$

$[1,2]$. Just as in the axisymmetric case (Chapter 2) the approximate effective reluctance is of the form: $c_{i}(s) \sqrt{s}+R_{i}^{0}$, where $R_{i}^{0}$ is the static reluctance and $c_{i}(s)$ represents the dynamic component of effective reluctance. Each of these regions are components of a magnetic circuit arranged in series. The approximate effective reluctance of a C-type actuator $(\widetilde{R})$ is given by the sum of the effective reluctance of each region:

$$
\widetilde{R}=\left(c_{s t}(s)+c_{f l}(s)+2 c_{g}(s)\right) \sqrt{s}+R_{s t}^{0}+R_{f l}^{0}+2 R_{g}^{0},
$$


where subscripts $s t$ represent the stator region, $f l$ represents the flotor region, and $g$ represents the air gap regions, and the perturbation flux is given by

$$
\phi_{p}(s)=\frac{N}{R} i_{p}(s)
$$

\subsection{Analytic Model for Segmented Thrust AMBs}

If it were possible to cut the flotor and stator then Zhu's analytic model for C-type actuator geometries could be used directly for each segment and the reluctance of each segment, which forms the symmetric branches of a parallel magnetic circuit, could be used to determine the total reluctance. However, as mentioned previously, a segmented flotor is not practical in rotating machinery due to mechanical strength requirements. Since the C-type model has separate reluctance terms for the flotor and stator the flotor may be modeled as a C-type flotor by neglecting the curvature and using the average circumference of the inner and outer poles as the length parameter. However, because C-type actuators have two free ends, this strategy assumes that the flotor has a single cut. It has been shown that if the length of a C-type actuator is much greater than the pole width then the electromagnetic field interactions at the ends of the actuator contribute little to the dynamics of the entire actuator [13,2]. Circumference for an axisymmetric geometry is analogous to length for a C-type geometry. Therefore, if the radius is large enough relative to other geometric parameters, then the effect of a single cut in the flotor will be negligible. Reluctance of the flotor is a small part of the total effective reluctance and reluctance of the air gaps is the dominant factor at low frequencies. As perturbation frequency increases, effective reluctance in the iron takes on a more dominant role. However, with a longer iron path in the stator relative to the flotor, stator cuts will have more influence on frequency which means that modeling an uncut flotor as having a single cut will have little impact on the result. In order to verify this statement, FEA was carried out to determine the effect of making a single cut in the floater for an uncut stator as well as for a stator with a single cut. Using Case 1 from Chapter 2 normalized flux magnitude and phase were analyzed for each of these cases. These results (Figure 3.6) show that a single flotor cut has little effect on flux magnitude, which is proportional to perturbation force, and phase validating the proposed strategy to model the flotor as a C-type flotor. As expected, FEA predicts that a single stator cut will have more impact on magnitude and phase than a single cut in the flotor.

Using Zhu's full analytic model of reluctance for the flotor, air gap, and stator regions where the reluctance is the product of a static term $\left(R^{0}\right)$ multiplied by a dynamic factor $\left(R^{d}\right)$,

$$
R_{i}=R_{i}^{0} R_{i}^{d}(s)
$$


the total reluctance of an actuator with $m$ stator segments in parallel may be expressed as:

$$
R=\frac{R_{s}^{0} R_{s}^{d}(s)}{m}+R_{f}^{0} R_{f}^{d}(s)+2 R_{g}^{0} R_{g}^{d}(s)
$$

The transfer function from perturbation current $\left(i_{p}\right)$ to perturbation flux $\left(\phi_{p}\right)$ becomes:

$$
\phi_{p}(s)=\frac{N}{\frac{R_{s}^{0} R_{s}^{d}(s)}{m}+R_{f}^{0} R_{f}^{d}(s)+2 R_{g}^{0} R_{g}^{d}(s)} i_{p}(s)
$$

The perturbation force $\left(F_{p}\right)$ can then be determined via the Maxwell stress tensor, $[3,10]$, to be:

$$
\begin{aligned}
f(t) & =\frac{1}{2 \mu_{0}} \oint_{S} \mathbf{B}^{2}(t, r) \mathrm{d} S \\
\text { For } B=B_{b}+B_{p}, f(t) & =\frac{1}{2 \mu_{0}} \oint_{S}\left[\mathbf{B}_{b}^{2}+2 \mathbf{B}_{b} \mathbf{B}_{p}(t, r)+\mathbf{B}_{p}^{2}(t, r)\right] \mathrm{d} S,
\end{aligned}
$$

such that for a small perturbation current, $\mathbf{B}_{p}^{2}(t, r) \approx 0$, and the first component of the force, $\frac{1}{2 \mu_{0}} \oint_{S} \mathbf{B}_{b}^{2} \mathrm{~d} S$, is static. Therefore, the dynamic component of the force is represented by the middle term in the Maxwell stress tensor (Equation 3.16). The Laplace transform of this term is given by:

$$
\begin{gathered}
\mathcal{L}\left[f_{p}(t)\right]=\mathcal{L}\left[\frac{1}{\mu_{0}} \oint_{S} \mathbf{B}_{b} \mathbf{B}_{p}(t, r) \mathrm{d} S\right] \\
F_{p}(s)=\frac{1}{\mu_{0}} \oint_{S} \mathbf{B}_{b} \mathbf{B}_{p}(s, r) \mathrm{d} S .
\end{gathered}
$$

Integrating flux density gives the total perturbation flux $\left(\phi_{p}\right)$. Since the largest component of perturbation force is given by the integral of flux density, rather than flux density squared, $\mathbf{B}_{p}=\frac{\phi_{p}}{A}$ can be substituted into Equation 3.17 to generate the transfer function representing the force output for a given perturbation flux input [2] as

$$
\begin{aligned}
F_{p}(s) & =\frac{1}{\mu_{0}}\left[\int_{r_{0}}^{r_{1}} \mathbf{B}_{b} \mathbf{B}_{p}(s, r) \cdot 2 \pi r \mathrm{~d} r+\int_{r_{2}}^{r_{3}} \mathbf{B}_{b} \mathbf{B}_{p}(s, r) \cdot 2 \pi r \mathrm{~d} r\right] \\
& =\frac{\phi_{b}}{\mu_{0}}\left(\frac{1}{A_{1}}+\frac{1}{A_{2}}\right) \phi_{p}(s)
\end{aligned}
$$


Substituting Equation 3.15 into Equation 3.18 results in the full analytic model for a perturbation force output for a given perturbation current for a thrust AMB with a segmented stator as:

$$
F_{p}(s)=\frac{\phi_{b}}{\mu_{0}}\left(\frac{1}{A_{1}}+\frac{1}{A_{2}}\right)\left[\frac{N}{\frac{R_{s}^{0} R_{s}^{d}(s)}{m}+R_{f}^{0} R_{f}^{d}(s)+2 R_{g}^{0} R_{g}^{d}(s)}\right] i_{p}(s)
$$

Using the approximate model for perturbation flux (Equation 3.12) the approximate half-order transfer function model for a segmented geometry with $m$ stator segments may be written as:

$$
F_{p}(s)=\frac{\phi_{b}}{\mu_{0}}\left(\frac{1}{A_{1}}+\frac{1}{A_{2}}\right) \frac{N}{c(s) \sqrt{s}+R^{0}} i_{p}(s),
$$

where the static and dynamic components of effective reluctance are given by:

$$
\begin{aligned}
R^{0} & =\frac{R_{s t}^{0}}{m}+R_{f l}^{0}+2 R_{g}^{0} \\
c(s) & =\frac{c_{s t}(s)}{m}+c_{f l}(s)+2 c_{g}(s)
\end{aligned}
$$

\subsection{3-D Finite Element Analysis}

Due to the disruption of axisymmetry by stator segmentation, 3-D FEA is required to analyze the effect of stator cuts. For benchmarking the 3-D ANSYS model, the uncut geometry with 64-fold symmetry was used for comparison of mesh resolutions with the 2-D FEMM results from Chapter 2 (Figure 2.8) as a reference. Disruption of the axisymmetry of the actuator by the introduction of stator cuts requires that larger wedges be modeled resulting in more nodes and larger FEA formulations. The worst case, from a computational perspective, is a 2 -fold symmetry model for a stator with 1 or 2 cuts. Stator cuts are modeled by an air gap or by using a parallel current boundary condition. FEA results shown here neglect leakage and fringing as does the analytic model.

Figure 3.7 shows a comparison of two mesh resolutions in ANSYS with the finest mesh resolution used in the 2-D FEA from Chapter 2, focusing on frequencies from 100 to $1000 \mathrm{~Hz}$. Flux magnitudes generated using ANSYS 3-D FEA were very close to the magnitudes from the 2-D model (Figure 3.7). However, the phase estimated using the standard mesh resolution in ANSYS, diverges from the results obtained using FEMM for frequencies above $100 \mathrm{~Hz}$, Fig. 3.7. A similar phenomenon was observed in Chapter 2 with the lower mesh resolutions matching the magnitude but not the phase. At higher frequencies, eddy-current

effects restrict the flux path to fewer elements near the material surface which is likely causing the errors 
observed at higher frequencies. With 2-D FEA this problem is easily addressed by increasing the mesh resolution. However, with 3-D modeling, increasing the mesh resolution presents a computational challenge. Using a finer 3-D mesh, the phase results at $400 \mathrm{~Hz}$ are very close to matching the 2-D results from FEMM. However, due to the excessively large times required for computation, up to $72 \mathrm{hr}$ for a single frequency for the 64-fold symmetry model, it was not deemed practical to use the finer mesh resolution for FEA of segmented geometries. Instead the mesh resolution was limited, dependent on the geometry, and the slight phase error was deemed acceptable. For each case, the mesh resolution used was that which could be solved in a 7 day time period for the worst-case symmetry.

Plots of flux distribution for Case 1, generated using ANSYS, clearly show the effect of stator segmentation (Figure 3.8). Actuators with an uncut stator and a stator with 16 cuts were driven with an amplitude of $1 \mathrm{~A}$ at frequencies of $3 \mathrm{~Hz}$ and $50 \mathrm{~Hz}$. The magnitude of the flux distributions generated by ANSYS are shown in Figure 3.8. With an uncut stator, eddy currents are more severe making the skin effect more apparent with higher flux densities close to the iron surface. However, with a segmented stator, the eddy current loops circumscribing the stator are broken, restricting eddy currents to smaller regions. Smaller eddy current loops reduce the effective reluctance in the iron which leads to a more uniform flux distribution. The effect this has on dynamic performance is significant, as will be shown in the remainder of this chapter.

Normalized magnitude (Equation 2.41) and phase of the perturbation force were calculated by FEA for perturbation frequencies from 3 to $400 \mathrm{~Hz}$ (Figure 3.9). With a single stator cut, a significant shift in magnitude is observed, with smaller shifts for additional cuts (Figure 3.9). FEA also shows a phase improvement with an increasing number of stator cuts (Figure 3.9). These results demonstrate the potential for dynamic performance enhancements as a result of stator segmentation.

For frequencies above $100 \mathrm{~Hz}$, the phase lag predicted by 3-D FEA is an overestimate. This is obvious when comparing ANSYS results with those from FEMM for the uncut geometry (Figure 3.9). However, by comparing only 3-D FEA results it may be concluded, assuming the phase error at high frequencies is similar for each number of stator cuts, that a significant reduction in phase lag will occur (Figure 3.9). Using several test cases FEA was used to verify the analytic model presented in Section 3.3.

\subsection{Verification of the Analytic Model}

To verify the analytic model for segmented thrust bearings developed in Section 3.3, the same test cases used in Chapter 2, Table 2.2, are used as the uncut base geometries. The first test case is based on an existing thrust bearing on the compressor surge test rig developed at the University of Virginia [24, 25]. Additional test cases present variations in coil width, height, air gap, and inner diameter. Each geometry maintains 
the same pole face area. The pertubation force estimated by the analytic model, full and approximate, was compared to the results obtained by 3-D FEA.

Adaptation of a segmented thrust AMB to an analytic model developed for C-type geometries requires a choice of pole parameters, length and width, since these values differ for inner and outer poles of a thrust AMB. The simple solution is to use the average of the inner and outer pole length and width. This solution will provide accurate results for most thrust bearing geometries. However, for thrust AMBs with extreme ratios of pole face area, $A_{p}=\pi\left(r_{1}^{2}-r_{0}^{2}\right)=\pi\left(r_{3}^{2}-r_{2}^{2}\right)$, to coil groove area, $A_{c g}=\pi\left(r_{2}^{2}-r_{1}^{2}\right)$, using the average values of pole length and width is not an adequate solution. Exteme geometries are likely to be rare in industrial applications, but were included here as a test of the modeling strategy. Weighting parameters for adaptation of the inner and outer pole dimensions to the C-type analytic model are given in Table 3.1 where $2 a$ is the adapted pole length, $2 b$ is the adapted pole width, $W_{a}$ is the weighting parameter for pole length, and $W_{b}$ is the weighting parameter for pole width. In general, for $0.5<\frac{A_{p}}{A_{c g}}<0.6$ using the average of the inner and outer pole length and width resulted in a generally accurate approximation. For $\frac{A_{p}}{A_{c g}}<0.5$, $W_{a}=0.1$ and $W_{b}=0.9$ and for $\frac{A_{p}}{A_{c g}}>0.6, W_{a}=1.0$ and $W_{b}=0$ generally produced the best results. If necessary, the pole width parameter may be tuned to match the static force predicted by FEA as the shape of the frequency response is not sensitive to changes in $b$. Results are not highly sensitive to $a$ either, which is the reason why three sets of values can be recommended for a wide range of geometries.

$$
\begin{aligned}
& 2 a=\pi\left[\left(1-W_{a}\right)\left(r_{0}+r_{1}\right)+W_{a}\left(r_{2}+r_{3}\right)\right] \\
& 2 b=\left(1-W_{b}\right)\left(r_{3}-r_{2}\right)+W_{b}\left(r_{1}-r_{0}\right)
\end{aligned}
$$

Table 3.1: Guidlines for adaptation of segmented thrust AMB geometry to C-type analytic model.

\begin{tabular}{lccc} 
Case & $\frac{A_{p}}{A_{c g}}$ & $W_{a}$ & $W_{b}$ \\
\hline 1 & 0.55 & 0.5 & 0.5 \\
2 & 0.55 & 0.5 & 0.5 \\
3 & 0.24 & 0.1 & 0.9 \\
4 & 0.55 & 0.5 & 0.5 \\
5 & 0.36 & 0.1 & 0.9 \\
6 & 0.76 & 1.0 & 0 \\
\hline
\end{tabular}

Using the recommendations outlined above, force was estimated by the analytic model for each of the six test cases. Normalized magnitude and phase for the analytic and FEA model are shown in Figures 3.10 through 3.15. For Case 1, FEA was carried out for 1, 2, 4, 8, and 16 cuts with current perturbation frequencies 
of $0,3,6,12,25,50,100,200$, and $400 \mathrm{~Hz}$. For Cases 2 through 6, FEA analysis was only performed for 4, 8 , and 16 cuts with current perturbation frequencies of $0,3,12,50$, and $200 \mathrm{~Hz}$. In each case, the analytic model agrees very closely with the results of FEA in magnitude, and phase with the exception of the highest frequencies. At high frequency, the phase estimated using 3-D finite element analysis (ANSYS) is lower than that estimated by the analytic model. This is believed to be an artifact of the discretization in the finite element modeling that is unavoidable for the 3-D cases due to limited computational resources. As discussed in Section 3.4, this discrepancy in phase also appears in the comparison of the uncut geometry when comparing the results of 3-D and 2-D FEA. Therefore, the phase results of the analytic model at high frequency is potentially more accurate than the FEA results. These results suggest that the analytic model developed above for segmented thrust AMBs works very well for most thrust bearing geometries likely to be encountered in practice. However, accuracy cannot be guaranteed for geometries with a $\frac{A_{p}}{A_{c g}}$ ratio far outside the range of the test cases presented here. Due to computational limitations, flux leakage and fringing were not analyzed for segmented stator geometries.

\subsection{Dynamic Performance Enhancement by Stator Segmentation}

For Case 1, bandwidth predictions were made based on the full analytic model and FEA. Close agreement can be seen for each model, further supporting the accuracy of the analytic model. For the remaining cases, bandwidth was predicted based on the analytic model results only due to the reduced set of FEA-based frequency response data generated for these cases. It should be noted that for this work bandwidth was defined as the $-3 \mathrm{~dB}$ point.

Plotting the bandwidth as a function of stator cuts shows that the first stator cut has the greatest impact on performance (Fig. 3.16). The relationship between bandwidth and cuts appears to be affine for 1 to 16 cuts (Fig. 3.16) with cuts beyond the first resulting in a smaller bandwidth improvement. With 8 cuts in the stator, well within manufacturing and material constraints, a 2- to 2.5-fold increase in bandwidth is predicted for each case. These results confirm the hypothesis that stator segmentation leads to significant performance enhancements for non-laminated thrust AMBs.

\subsection{Flotor Segmentation}

As discussed in Section 3.3, adaptation of the C-type analytic model to segmented actuators requires that at least one cut is modeled for the flotor and stator. At low perturbation frequencies, cuts in the stator or flotor do not have much impact on force output. As perturbation frequency increases, eddy current effects, and thus 
the effects of segmentation, become more apparent. Because the flotor has a shorter iron path length than the stator, it is expected that cuts in the stator will impact dynamic performance more than cuts in the flotor.

FEA (Figure 3.6) showed that for an uncut stator and for a stator with a single cut, cutting the flotor had very little impact on force magnitude and phase output. However, the effect of more than one flotor cut has not been considered. Using the full analytic model, the effect of segmenting both the flotor and stator was compared to a segmented stator with a single cut in the flotor for Case 1. Results obtained using the analytic model are consistent with the results from FEA for low numbers of flotor cuts. Figure 3.17 shows that with two cuts or eight cuts in the stator, having a single cut in the flotor versus the same number of flotor and stator cuts has little impact on force output. However, as the number of stator and flotor cuts approaches 16, the dynamic effect of cutting the flotor along with the stator increases.

Figure 3.18 shows the corresponding bandwidths predicted by the full analytic model for a given number of stator cuts with either one flotor cut or the same number of cuts in the flotor and stator. It is clear from this analysis that a small number of flotor cuts has very little impact on performance but with more than eight cuts in the stator, having the same number of cuts in the flotor is predicted by the full analytic model to increase bandwidth significantly. While high stress levels in the flotor make segmentation impractical for high speed machines. These results suggest that if these difficulties could be overcome, or in the case of low operating speed, flotor and stator segmentation combined would provide better dynamic performance than stator segmentation alone. 

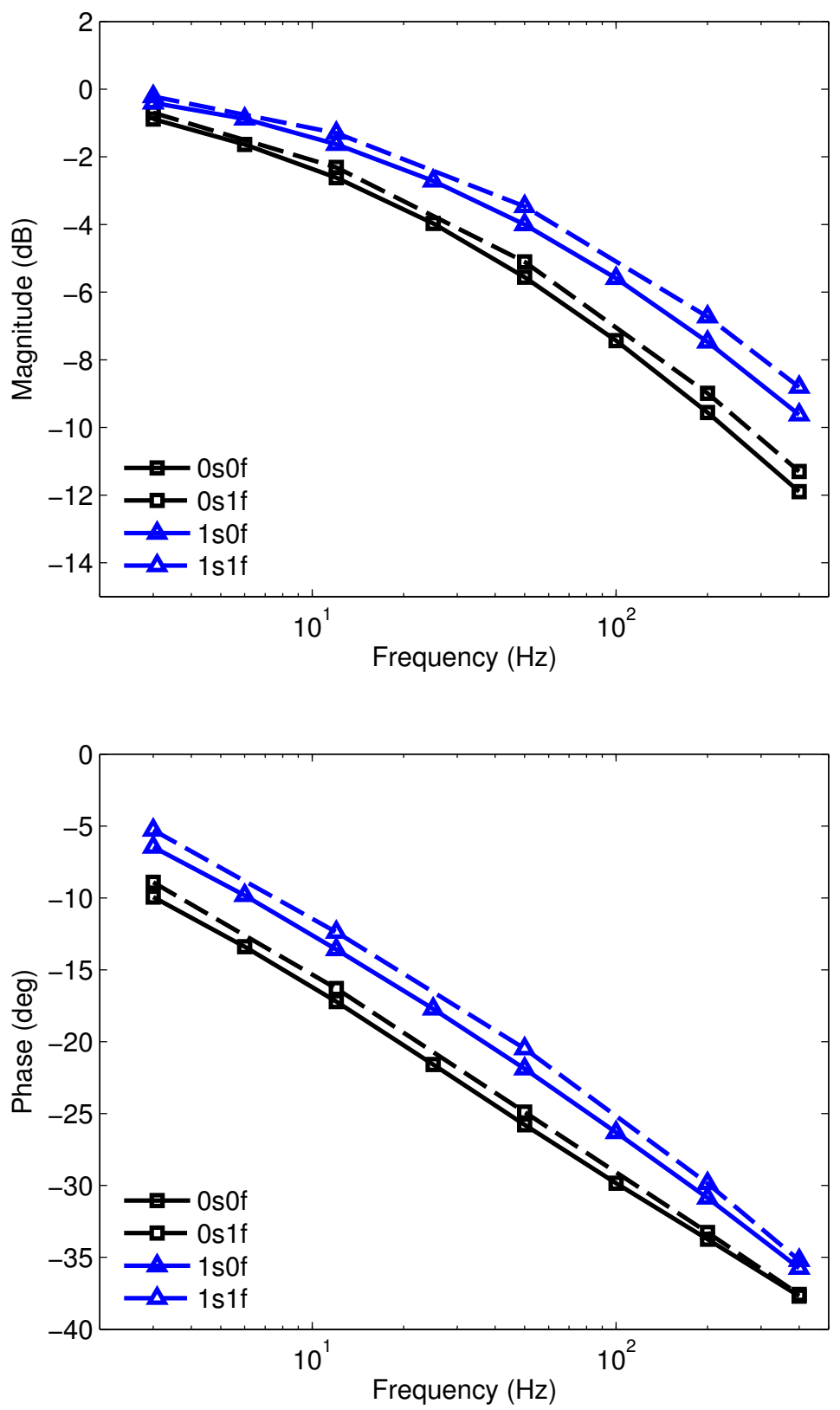

Figure 3.6: 3-D FEA results showing normallized flux magnitude and phase for an uncut stator with a flotor with 0 (0s0f) or 1 cut (0s1f) and a stator with a single cut with flotor with 0 (1s0f) or 1 cut (1s1f). 


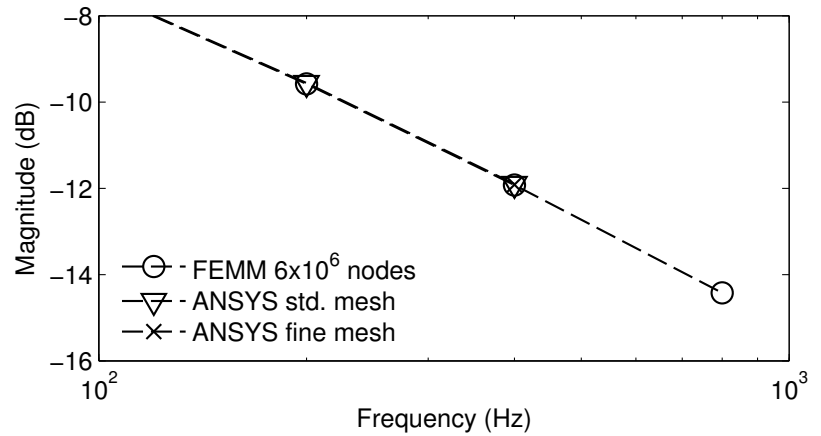

Magnitude: 3-D mesh analysis

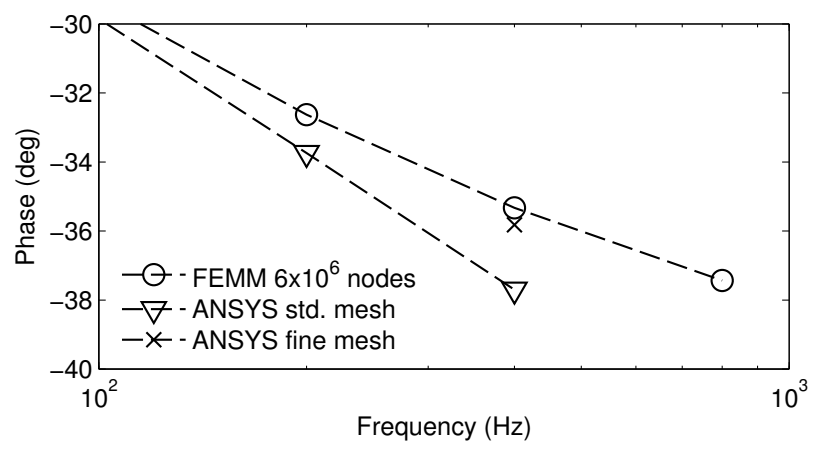

Phase: 3-D mesh analysis

Figure 3.7: Finite element modeling with perturbation current input and magnetic flux output shows magnitude and phase for a standard and fine mesh resolutions. 2-D FEA results, FEMM, are included as a reference. 


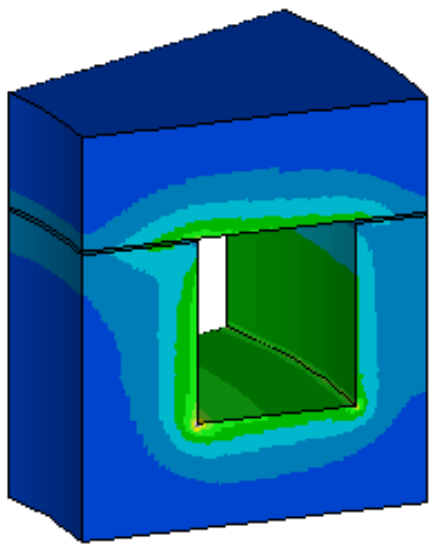

uncut stator

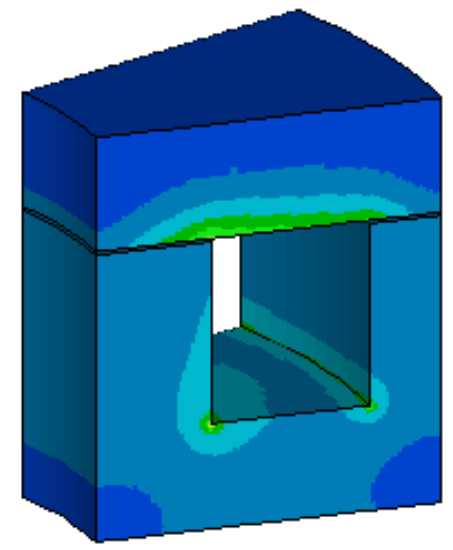

16 stator cuts

$3 \mathrm{~Hz}$ perturbation frequency

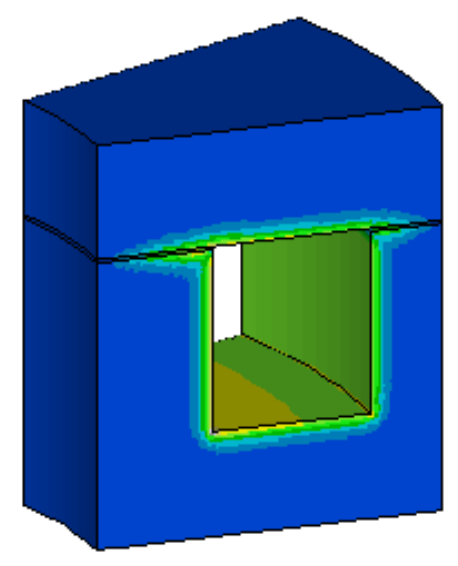

uncut stator

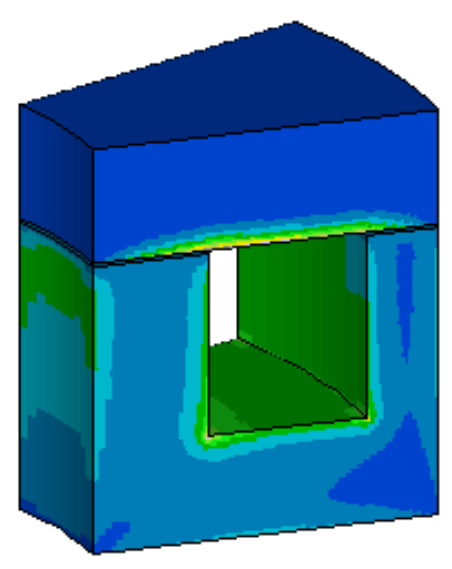

16 stator cuts

$0 \mathrm{~T}$

$1.4 \mathrm{~T}$

$50 \mathrm{~Hz}$ perturbation frequency

Figure 3.8: Magnetic flux density magnitude for a stator, case 1, with 0 or 16 cuts. The flotor is not cut. 

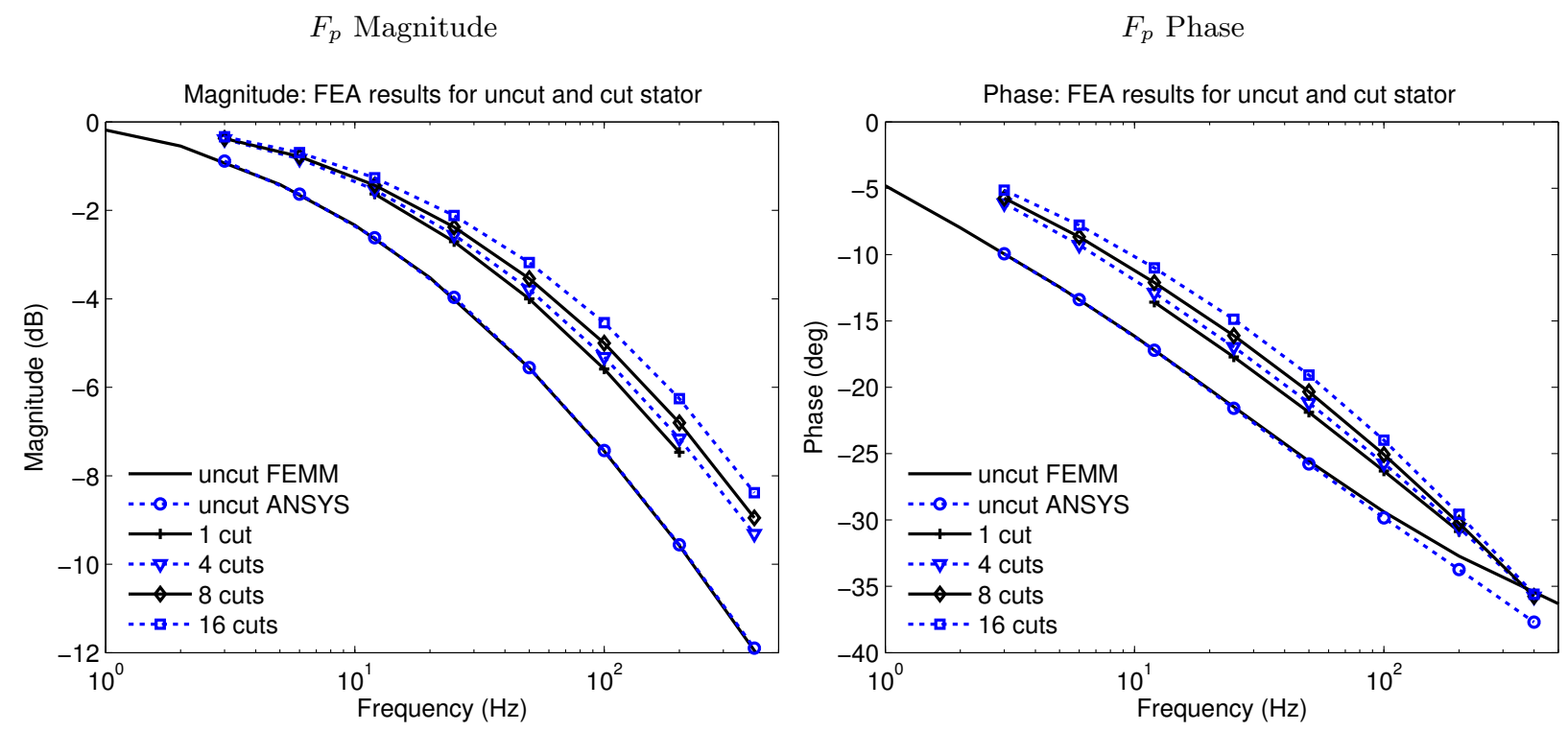

Figure 3.9: FEA Magnitude and phase from of thrust bearing, case 1, with unsegmented stator or cut stator. 
1 Stator Cut: Magnitude

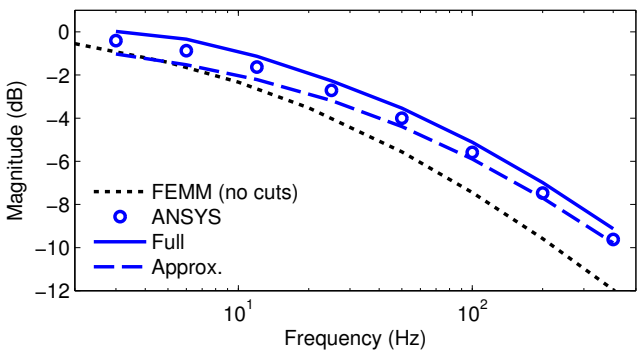

2 Stator Cuts: Magnitude

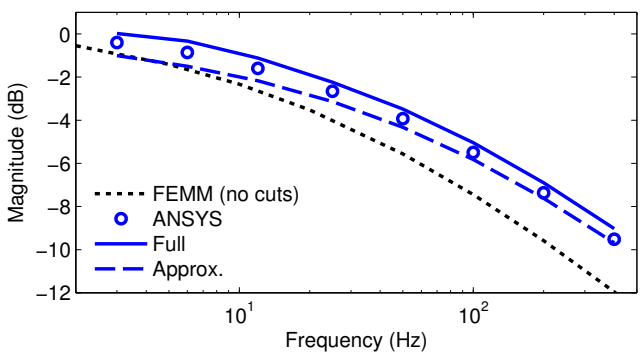

4 Stator Cuts: Magnitude

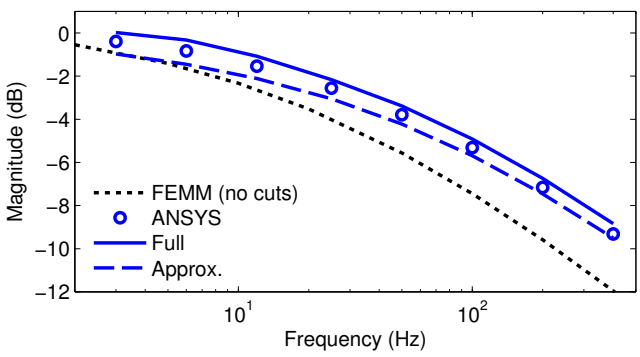

8 Stator Cuts: Magnitude

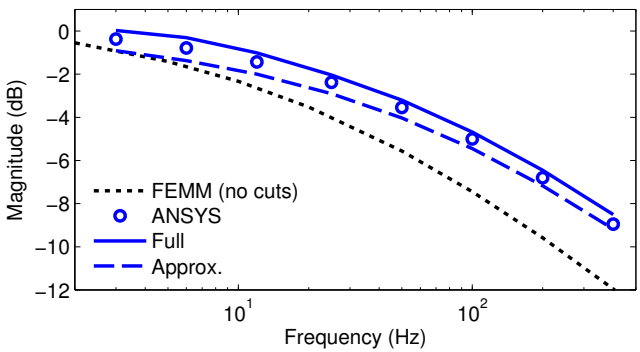

16 Stator Cuts: Magnitude

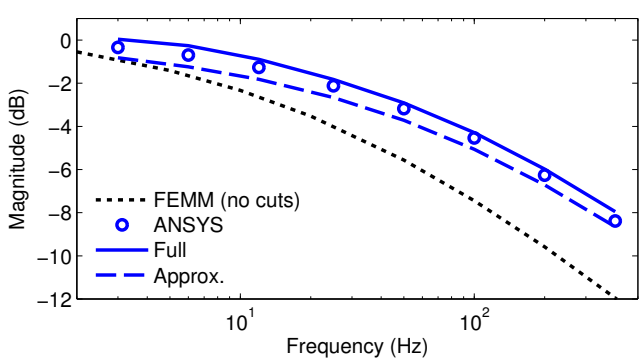

1 Stator Cut: Phase

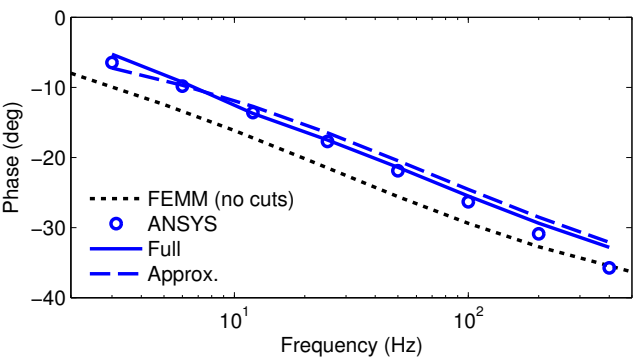

2 Stator Cuts: Phase

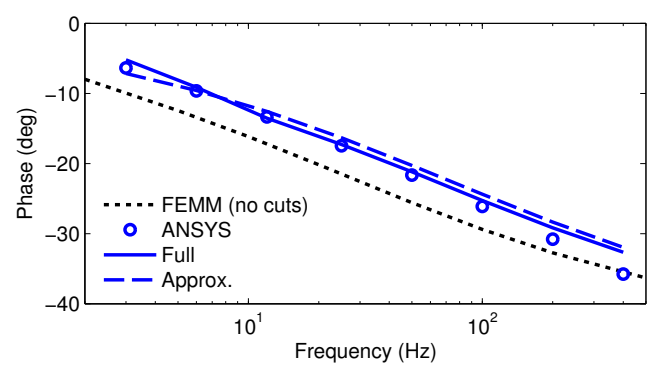

4 Stator Cuts: Phase

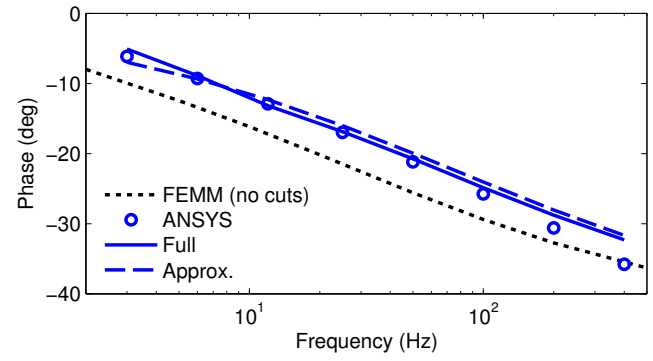

8 Stator Cuts: Phase

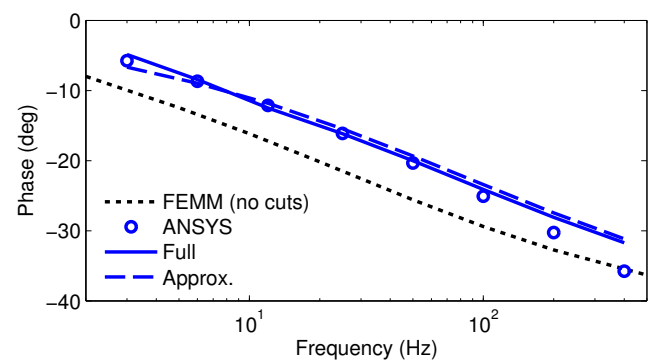

16 Stator Cuts: Phase

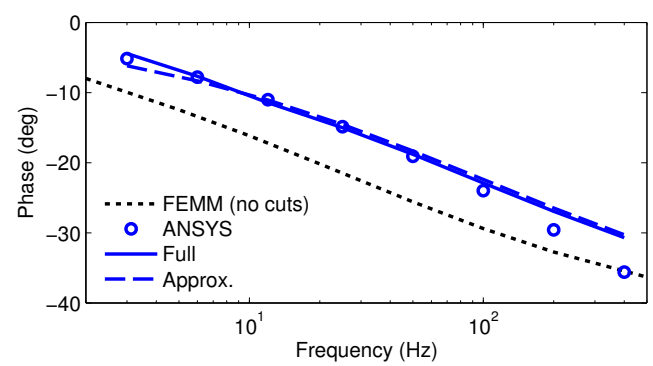

Figure 3.10: Case 1 (compressor thrust AMB) - frequency response for 1 to 16 stator cuts. 
4 Stator Cuts: Magnitude

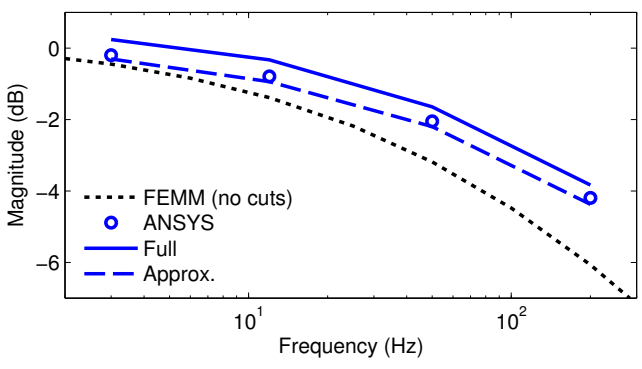

8 Stator Cuts: Magnitude

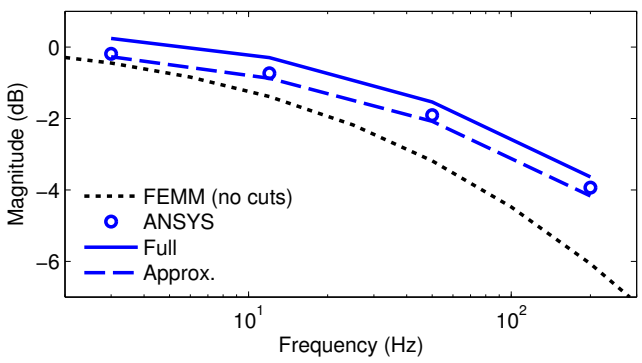

16 Stator Cuts: Magnitude

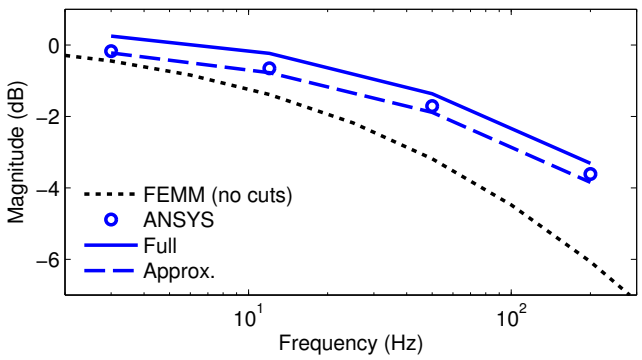

4 Stator Cuts: Phase

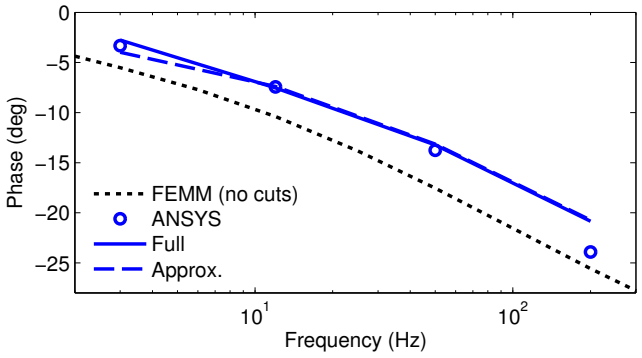

8 Stator Cuts: Phase

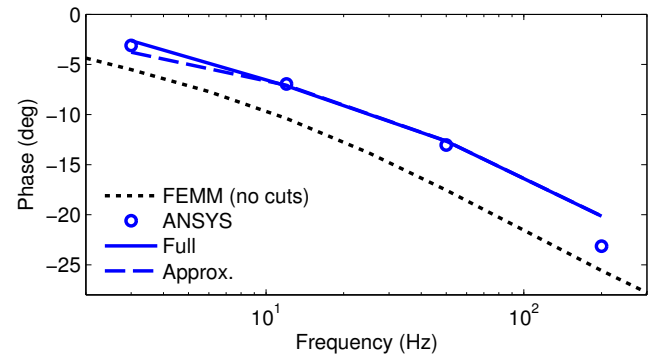

16 Stator Cuts: Phase

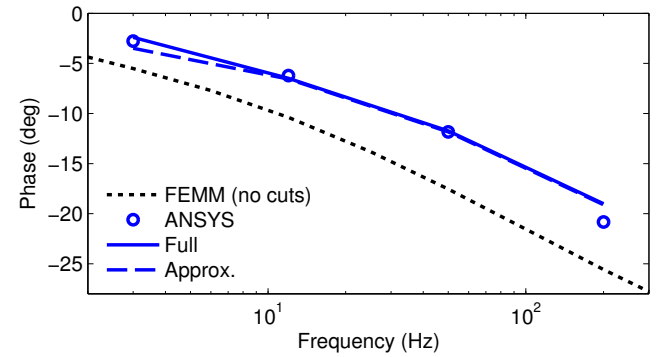

Figure 3.11: Case 2 (1mm air gap) - frequency response for 1 to 16 stator cuts. 
4 Stator Cuts: Magnitude

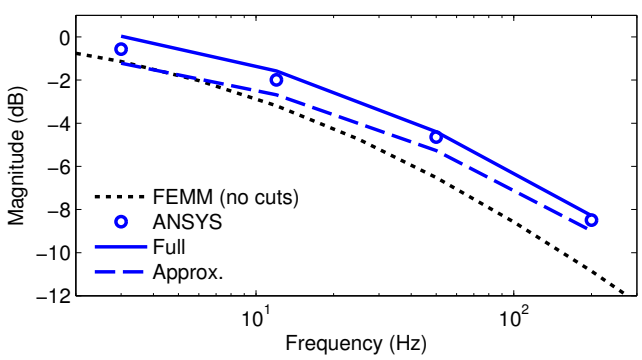

8 Stator Cuts: Magnitude

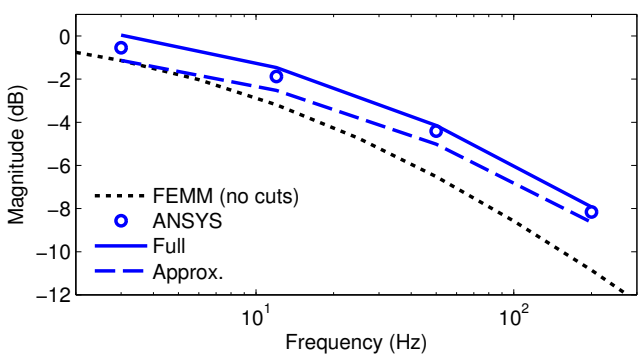

16 Stator Cuts: Magnitude

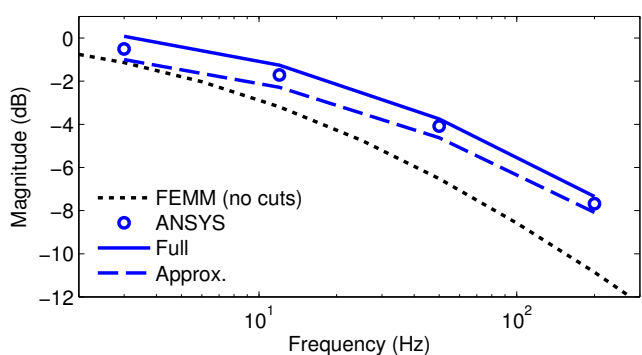

4 Stator Cuts: Phase

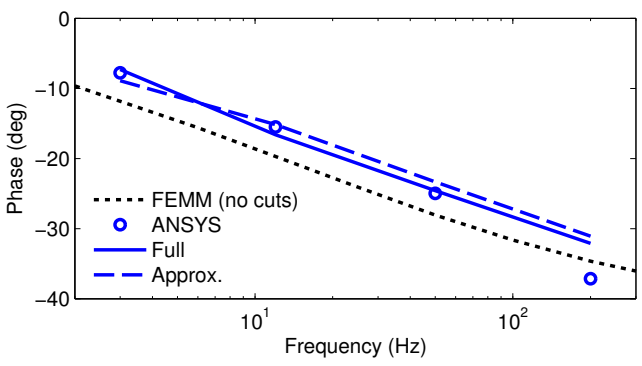

8 Stator Cuts: Phase

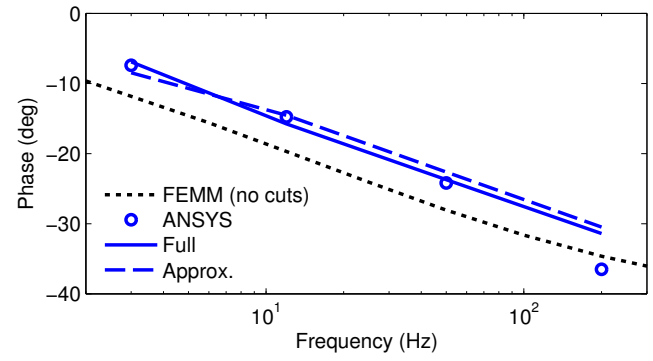

16 Stator Cuts: Phase

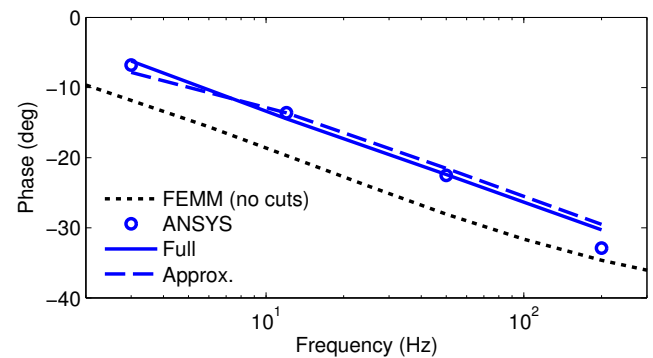

Figure 3.12: Case 3 (wide coil groove) - frequency response for 1 to 16 stator cuts. 
4 Stator Cuts: Magnitude

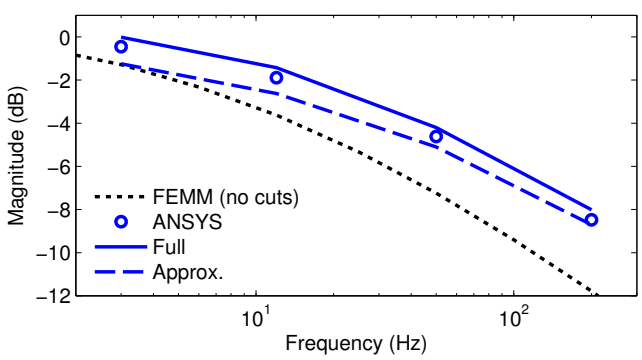

8 Stator Cuts: Magnitude

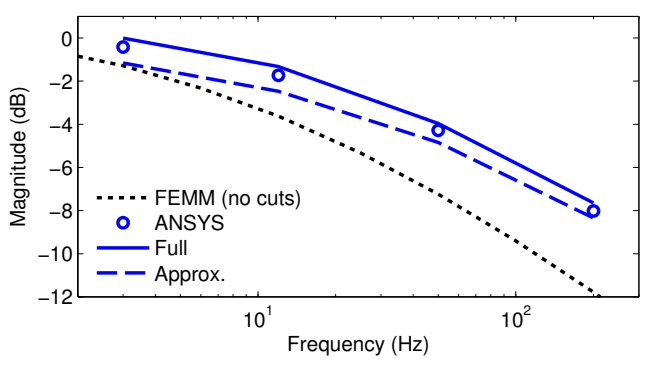

16 Stator Cuts: Magnitude

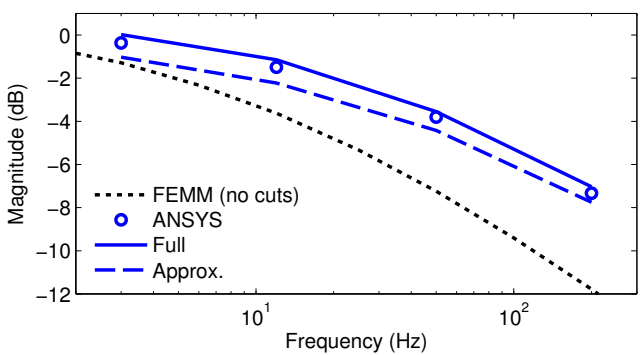

4 Stator Cuts: Phase

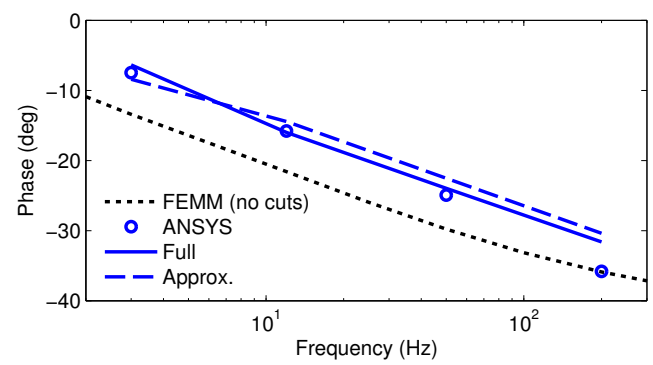

8 Stator Cuts: Phase

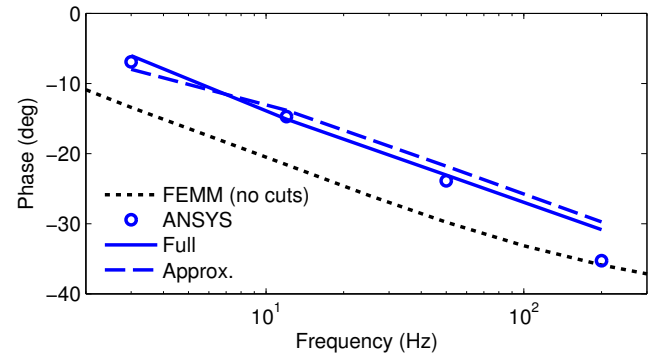

16 Stator Cuts: Phase

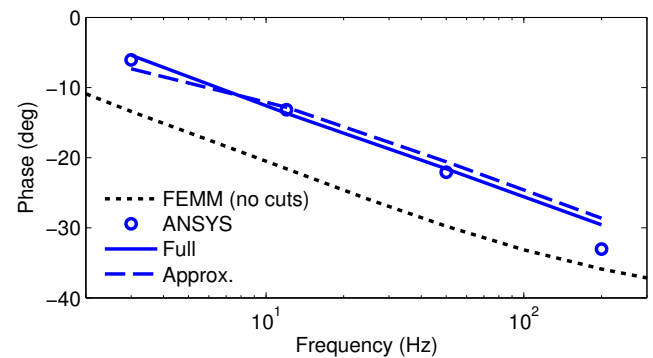

Figure 3.13: Case 4 (deep coil groove) - frequency response for 1 to 16 stator cuts. 
4 Stator Cuts: Magnitude

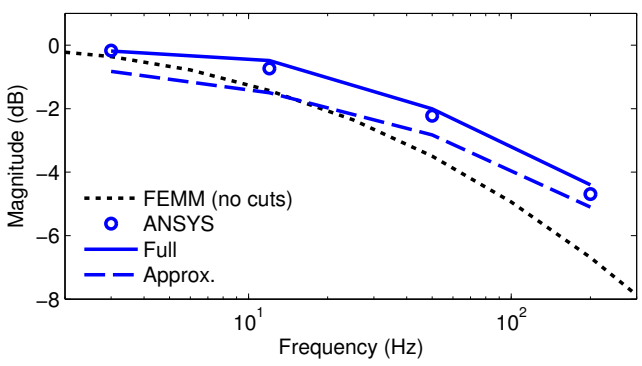

8 Stator Cuts: Magnitude

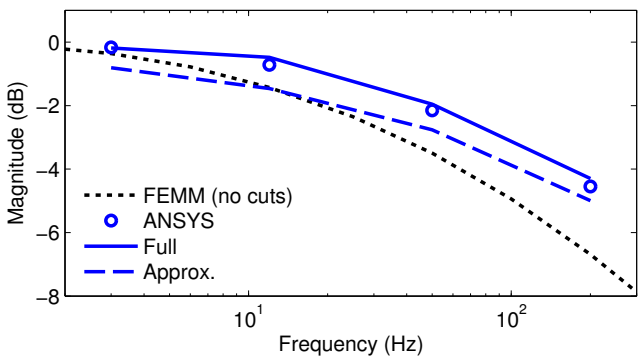

16 Stator Cuts: Magnitude

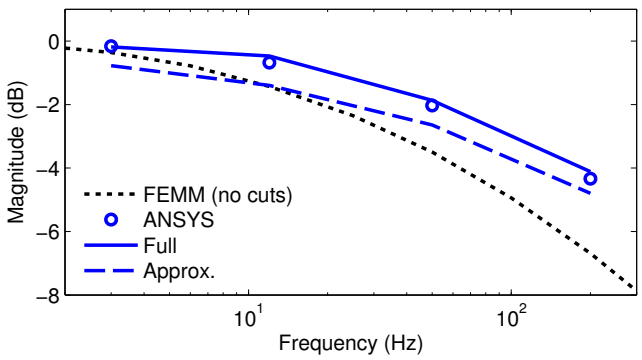

4 Stator Cuts: Phase

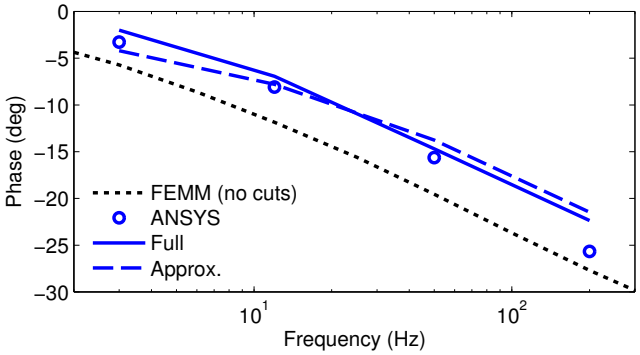

8 Stator Cuts: Phase

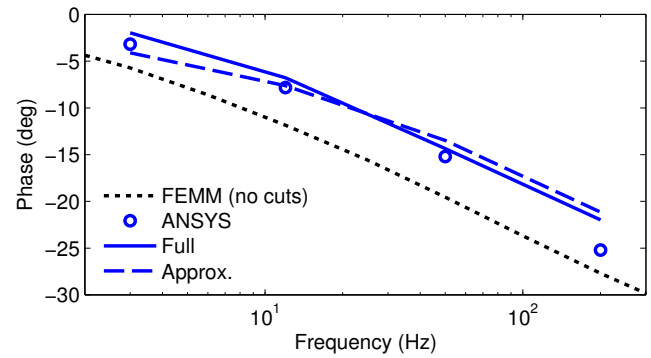

16 Stator Cuts: Phase

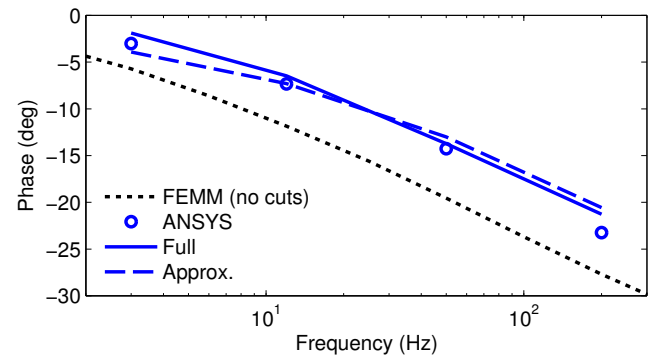

Figure 3.14: Case 5 (large inner radius) - frequency response for 1 to 16 stator cuts. 
4 Stator Cuts: Magnitude

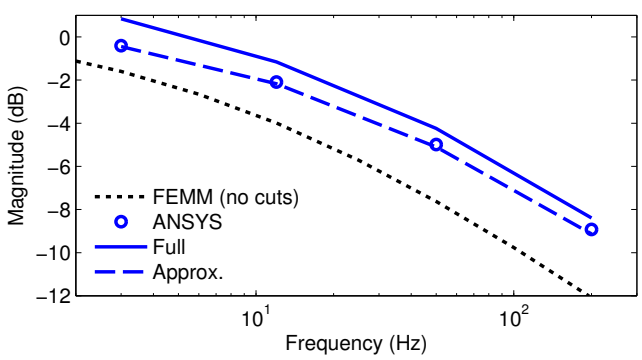

8 Stator Cuts: Magnitude

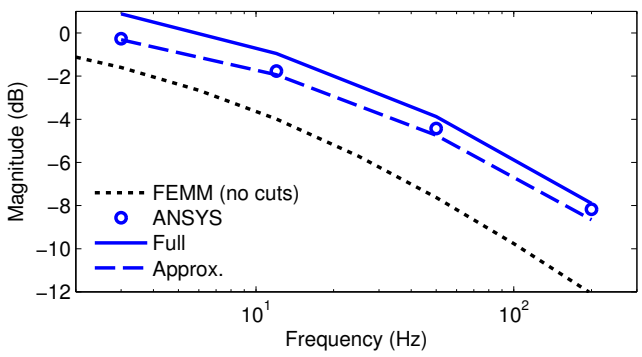

16 Stator Cuts: Magnitude

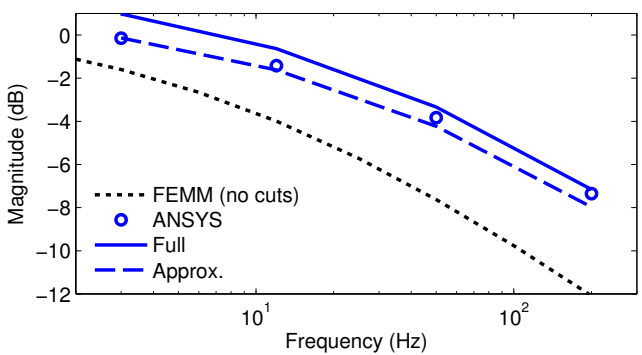

4 Stator Cuts: Phase

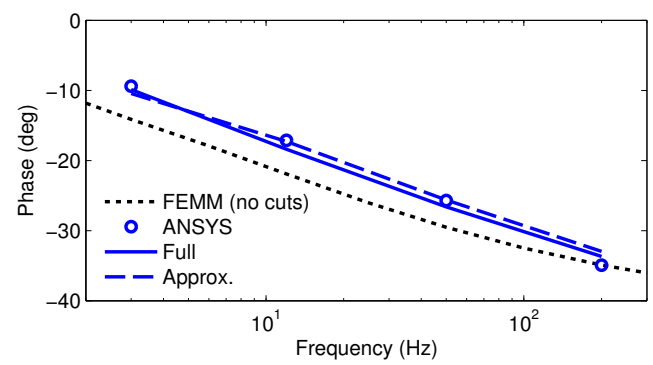

8 Stator Cuts: Phase

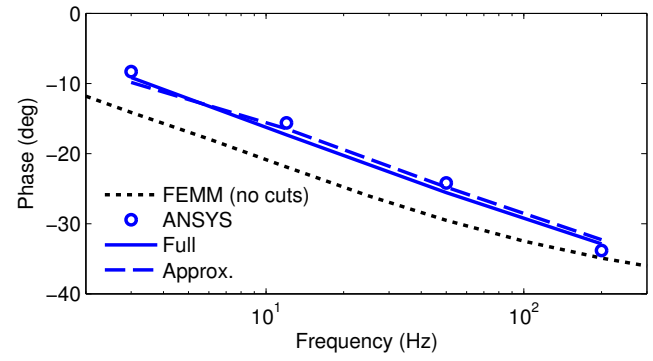

16 Stator Cuts: Phase

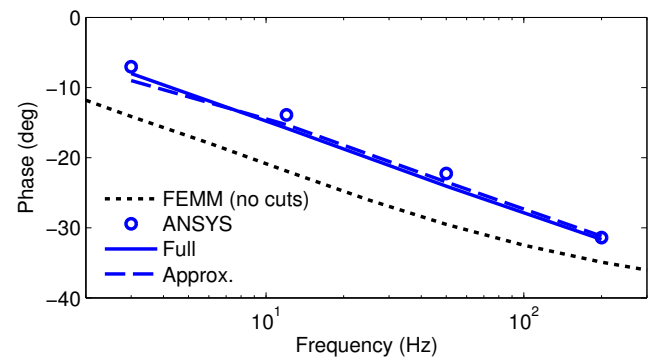

Figure 3.15: Case 6 (small inner radius) - frequency response for 1 to 16 stator cuts. 
Case 1 (compressor thrust AMB)

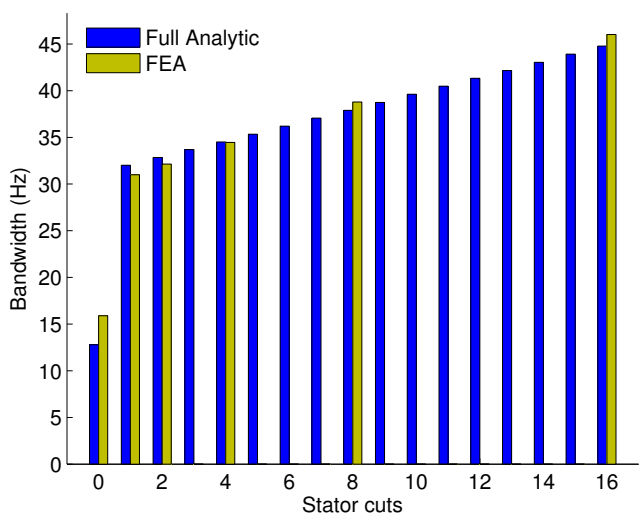

Case 3 (wide coil groove)

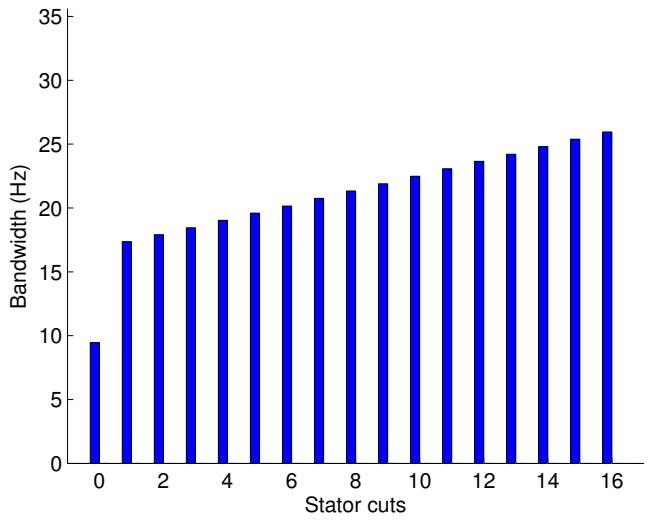

Case 5 (large inner radius)

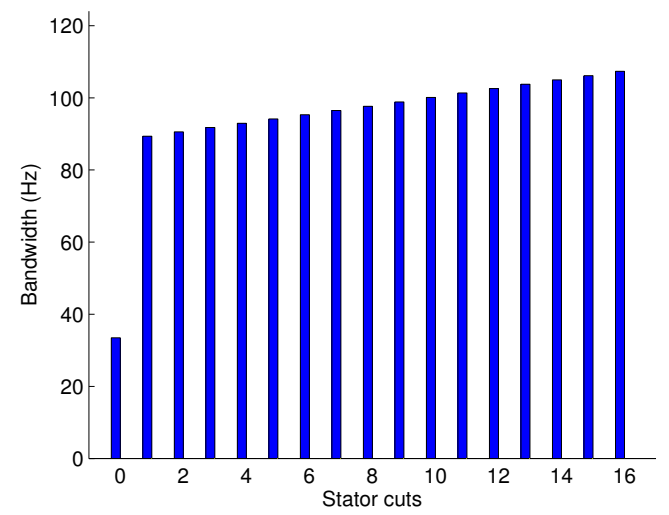

Case 2 (1mm air gap)

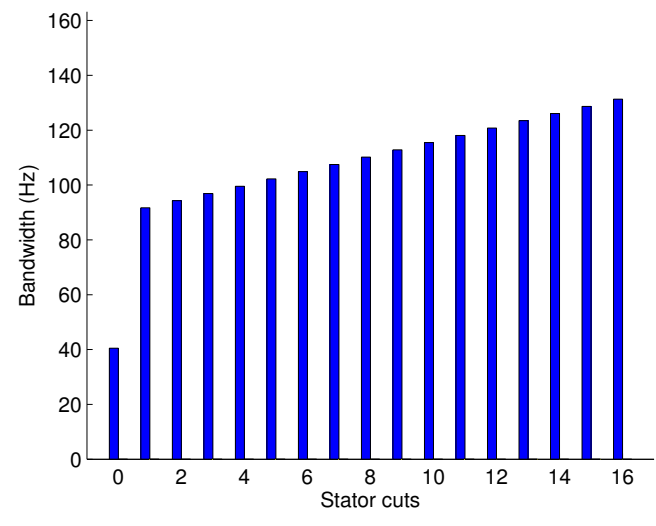

Case 4 (deep coil groove)

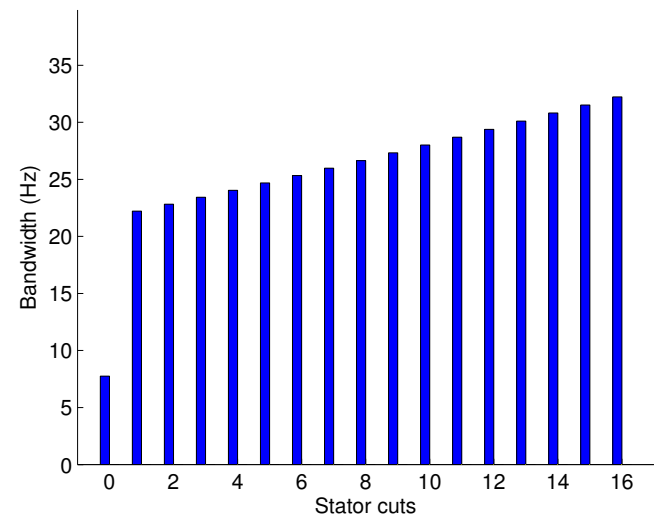

Case 6 (small inner radius)

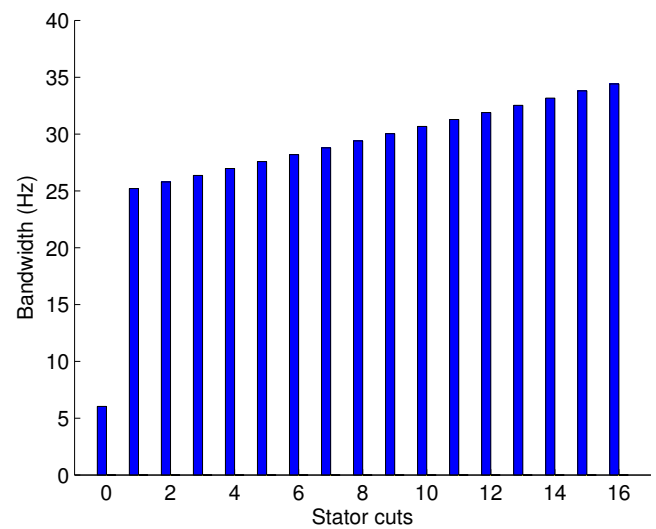

Figure 3.16: Bandwidth as a function of stator cuts, for each test case. 
2 Stator Cuts: Magnitude

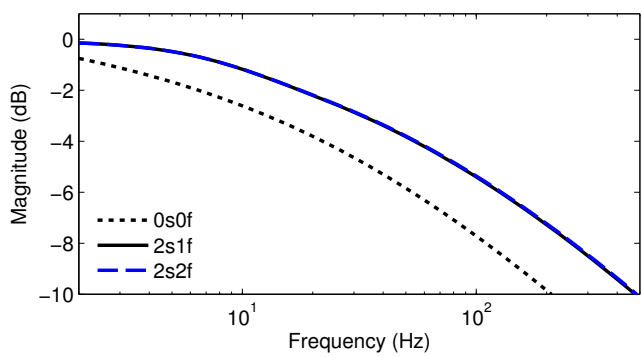

4 Stator Cuts: Magnitude

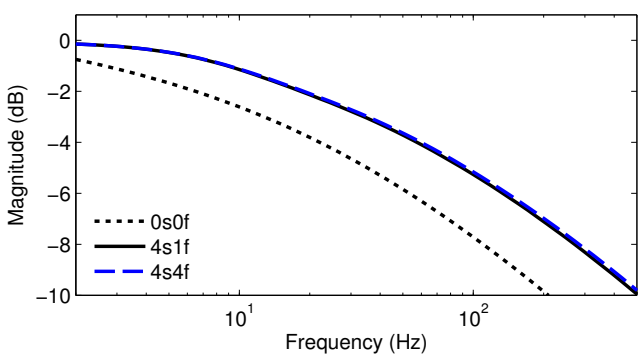

8 Stator Cuts: Magnitude

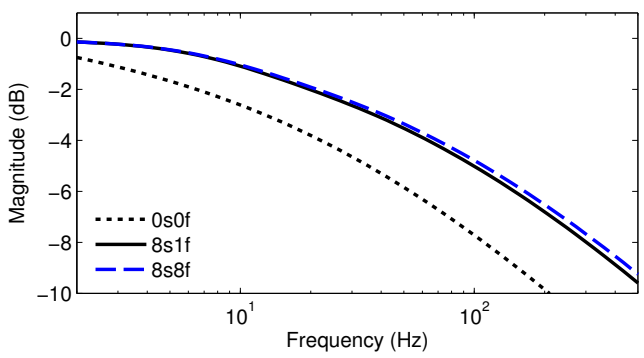

16 Stator Cuts: Magnitude

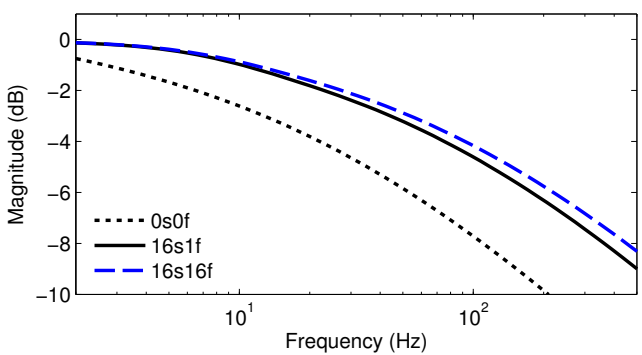

2 Stator Cuts: Phase

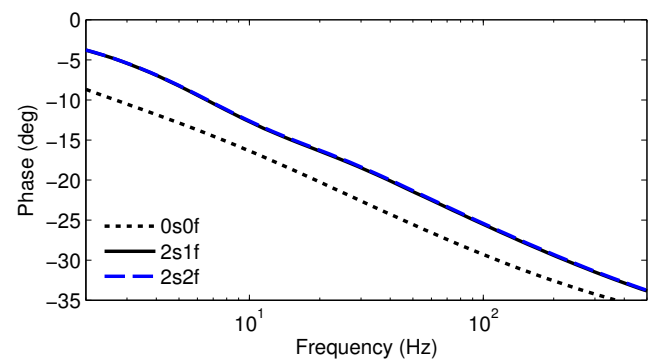

4 Stator Cuts: Phase

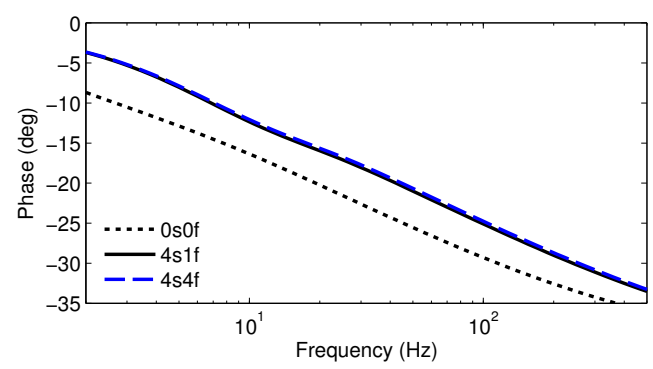

8 Stator Cuts: Phase

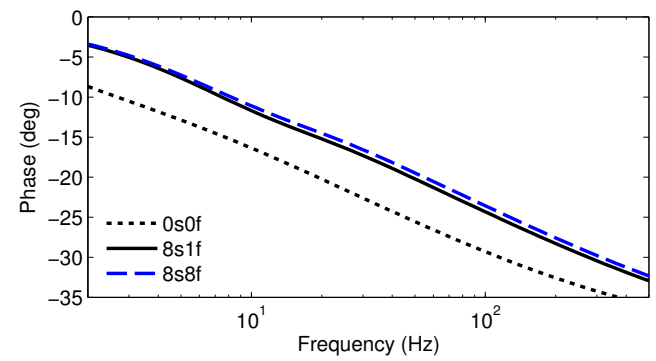

16 Stator Cuts: Phase

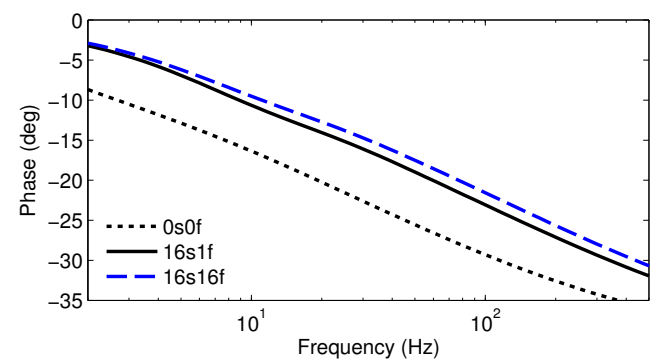

Figure 3.17: Analytic analysis, using the full model, of the effect of a single flotor cut on dynamic performance of Case 1 with 2, 4, 8, and 16 cuts. Labels nsmf correspond to analysis of an actuator with $\mathrm{n}$ stator cuts and $\mathrm{m}$ flotor cuts. Uncut actuator is included as a reference (0s0f). 


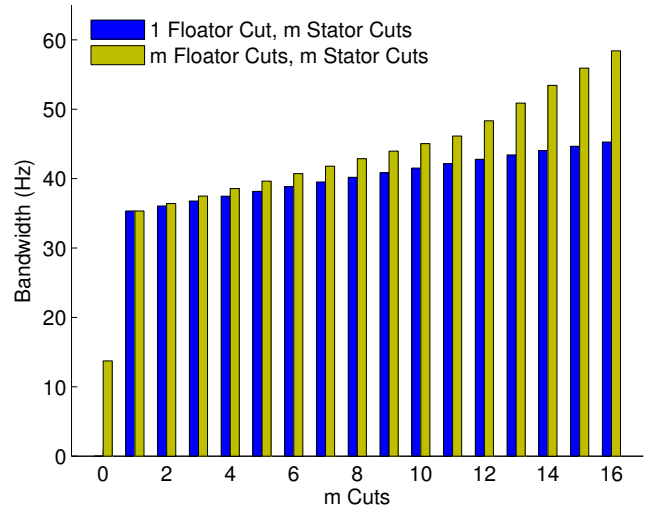

Figure 3.18: Bandwidth for Case 1 with a single flotor cut and 1 to 16 stator cuts compared to Case 1 with the same number of cuts in the flotor and stator. 


\section{Chapter 4}

\section{Control of Non-laminated AMBs Using Feedback Linearization}

The goal of this overall research effort is to develop tools that will enhance the performance of industrial machines with non-laminated AMBs. The work discussed in previous chapters has been directed towards greatly reducing the time and effort required for magnetic bearing design and development. Improved control design can also enhance performance for non-laminated AMB applications.

Magnetic bearings are highly nonlinear devices designed for a small range of operating conditions. For compressors subject to large axial disturbance forces, deviation from the nominal air gap degrades performance significantly. Low bandwidth associated with non-laminated thrust AMBs also degrades performance. Stator segmentation can improve bandwidth, as discussed in Chapter 3, but even with 16 stator segments the bandwidth will be much less than a laminated actuator. Ideally the controller would compensate for the effects of low bandwidth and a time-varying air gap. Feedback linearization (FBL) is a candidate control design technique capable of achieving this and has already been used successfully in laminated AMB systems to improve operating consistency over a wide range of air gaps. FBL may also prove capable of improving system performance by incorporating dynamic compensation of eddy current effects. The potential for performance improvement via feedback linearization will be addressed in this chapter. Based on detailed nonlinear simulation studies, it will be shown that (FBL) improves system response to unknown disturbance forces as well as improving low frequency reference tracking. 


\subsection{Feedback Linearization for Magnetic Bearings}

Nonlinear models of AMBs are typically linearized about a nominal air gap. This approach is generally well suited for AMBs since they typically operate near a single set point. However, in machines with large disturbance forces, or in the case of a novel surge control mechanism where servo control of the thrust AMB is used to cancel oscillations in pressure [24, 25], consistent operation over a wide range of displacements is desirable. Control by FBL seeks to cancel plant nonlinearities in order to improve performance consistency.

An AMB system with nonlinearity dependent on position in the air gap may be represented generally by the block diagram shown in Figure 4.1. Feedback linearization is common for this type of system and has been implemented successfully for AMB systems $[21,22,7]$ in voltage- and current-mode operation. Many industrial AMB systems are operated in current-mode which will be the focus of this work. Chen et al. demonstrated FBL for a laminated radial AMB in current-mode [7].

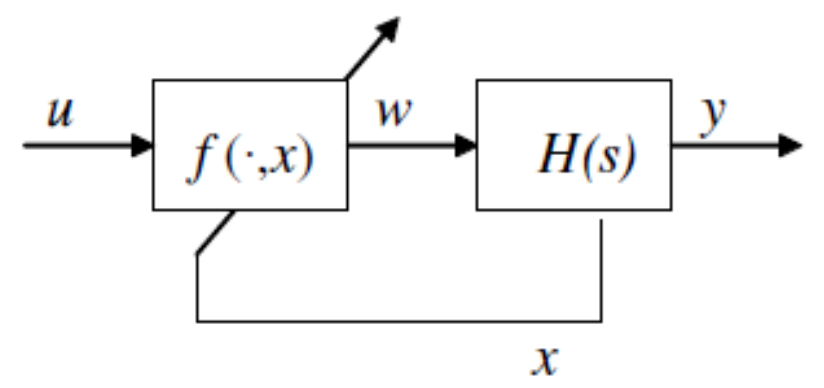

Figure 4.1: System with state-dependent nonlinearity.

Chen et al. determined the relationship between current, position, and force by experiment. This relationship was then represented by a Taylor series expansion of force around $I_{p, 0}=0$ and $x_{0}=0$ as

$$
f\left(I_{p}, x\right)=a_{0}+a_{1} I_{p}+a_{2} x+a_{3} I_{p}^{2}+a_{4} I_{p} x+a_{5} x^{2}+a_{6} I_{p}^{3}+a_{7} I_{p}^{2} x+\ldots
$$

However, instead of using the first three terms, as in traditional Jacobian linearization, a 5th order representation of the relationship was used and found to be highly accurate. A nonlinear compensator was then developed which generated the required current $\left(I_{p}\right)$ in order to determine the reference force $\left(F_{c}\right)$ output by a controller. The block diagram shown in Figure 4.2 depicts this arrangement graphically. In this case, for a given $F_{c}$ and $x$, multiple solutions exist for perturbation currents $\left(I_{p}\right)$ which satisfy the 5 th order Taylor series expression shown in Equation 4.1. However, the desired solution is real valued and less than the bias current. With these constraints, a unique 2-D lookup table was generated and used to implement the nonlinear compensation. 


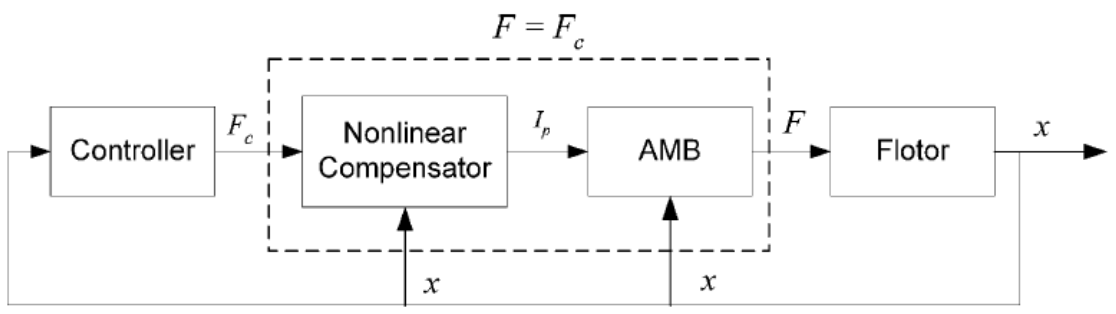

Figure 4.2: System with state-dependent nonlinearity [7].

In the example mentioned above, and in other examples of FBL for AMBs, the actuators were laminated and the FBL was static and based on experimental calibration. For non-laminated thrust bearings, it is proposed that nonlinear and dynamic compensation used in combination, offers a solution to improve not only performance consistency over the operating range but also to improve the bandwidth by compensating for eddy current effects. In addition, the accurate analytic models developed in Chapters 2 and 3 may prove adequate for the elimination of the need for time-consuming experimental calibration.

\subsection{Nonlinear Modeling}

For compensator development two nonlinear relationships were chosen as a focus: air gap to force and the full nonlinear Maxwell stress tensor which describes the relationship between flux density and force. In addition, the compensator will also include an inversion of the dynamic eddy current effect in an effort to extend the bandwidth. As a starting point, the model of a single-sided non-laminated actuator, including eddy current effects, developed by Zhu et al. [2] was used. Referring to Figure 4.3, the input is a perturbation voltage $\left(v_{p}\right)$ and the output is position $(z), R$ is coil resistance, and $K_{\phi}=\frac{1}{\mu_{0}}\left(\frac{1}{A_{1}}+\frac{1}{A_{2}}\right) \frac{N i_{b}}{R^{0}}$.

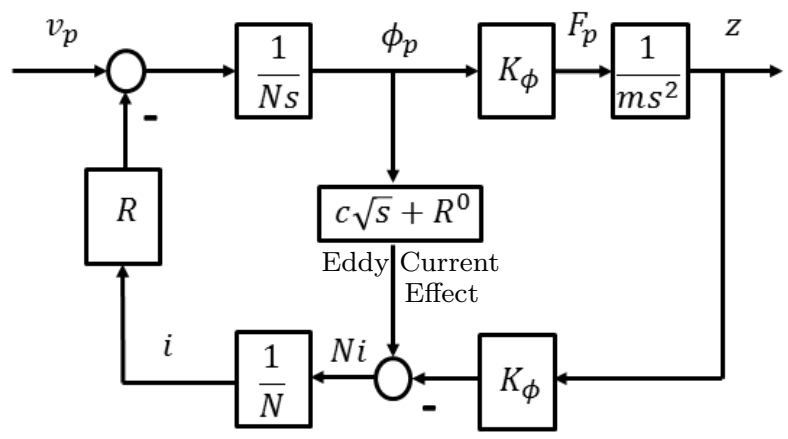

Figure 4.3: Block diagram representation of single-sided non-laminated magnetic actuator with voltage input.

The initial model (Figure 4.3) was modified to include the nonlinear relationships mentioned above. A transconductance amplifier was also added for operation in current-mode. Figure 4.4 shows the modified 
block diagram with the perturbation current reference input $\left(i_{p r e f}\right)$ and amplifier gain $\left(K_{a}\right)$ added. The block diagram was then rearranged to isolate the amplifier as an inner feedback loop (Figure 4.5). For large amplifier gain $\left(K_{a}\right)$ the inner loop is approximately equal to $N$ at low frequencies and may be simplified, along with the $1+\frac{R}{K_{a}}$ block in the outer feedback loop.

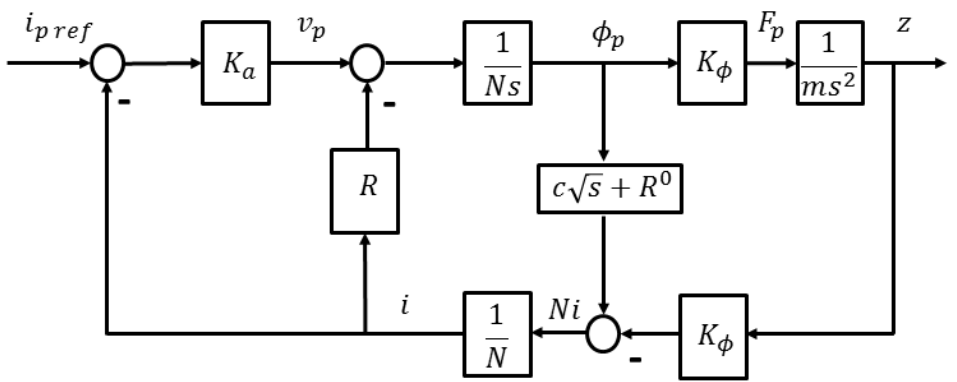

Figure 4.4: Non-laminated magnetic actuator with transconductance amplifier.

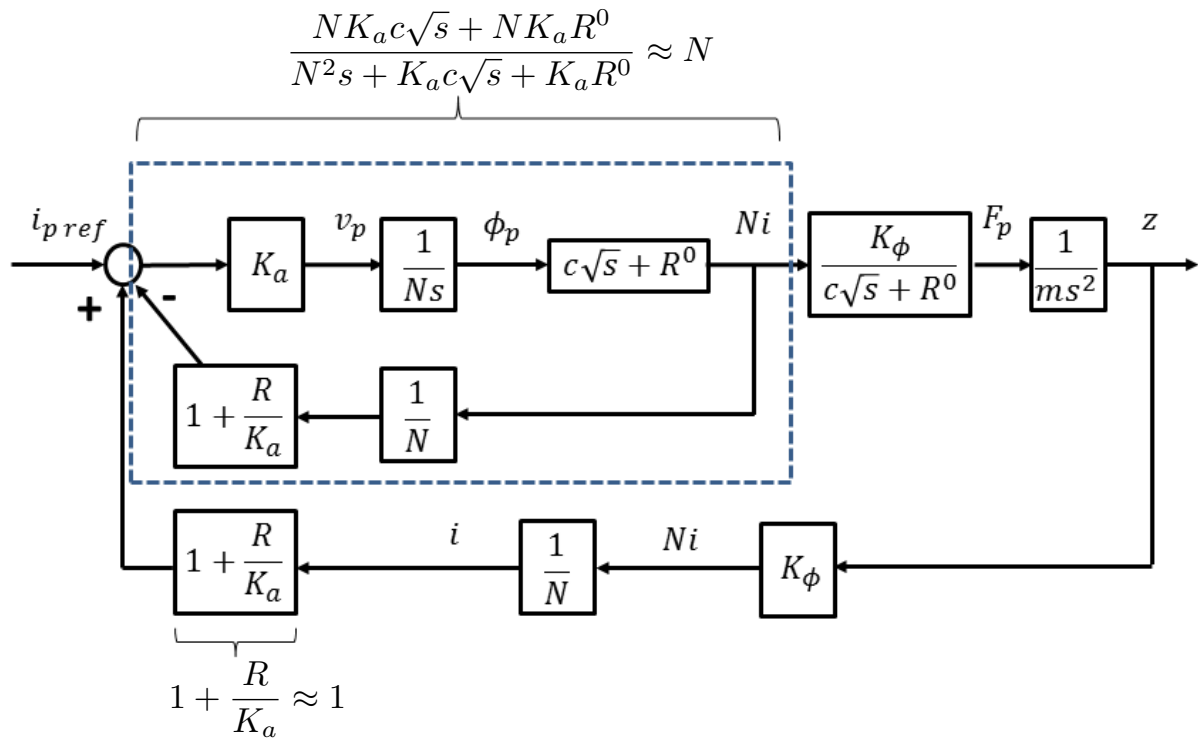

Figure 4.5: Non-laminated magnetic actuator with amplifier in a separate inner feedback loop.

Transfer functions relating current to force, developed in previous chapters, were based on an approximation of the Maxwell stress tensor, shown again in Equation 4.2 for convenience.

$$
\begin{aligned}
f(t) & =\frac{1}{2 \mu_{0}} \oint_{S} \mathbf{B}^{2}(t, r) \mathrm{d} S \\
& =\frac{1}{2 \mu_{0}} \oint_{S}\left[\mathbf{B}_{b}^{2}+2 \mathbf{B}_{b} \mathbf{B}_{p}(t, r)+\mathbf{B}_{p}^{2}(t, r)\right] \mathrm{d} S
\end{aligned}
$$


The approximation of the Maxwell stress tensor was based on the assumption that the perturbation current will be small relative to the bias current in which case the third term $\left(B_{p}^{2}(t, r)\right)$ of Equation 4.2 may be ignored. However, in high speed compressors, large axial disturbance forces will be accompanied by large perturbation currents and this approximation breaks down. Therefore, the full Maxwell stress tensor will be included in the nonlinear model for compensation as shown in Figure 4.6, where $F_{i}$ represents the three components of force given by Equation 4.2 and $f\left(\phi_{p}\right)$ represents the relationship between perturbation flux and the third force component of the Maxwell stress tensor $\left(F_{3}\right)$ given by:

$$
f\left(\phi_{p}\right)=\frac{1}{\mu_{0}} \oint_{S}\left[\mathbf{B}_{p}^{2}(t, r)\right] \mathrm{d} S
$$

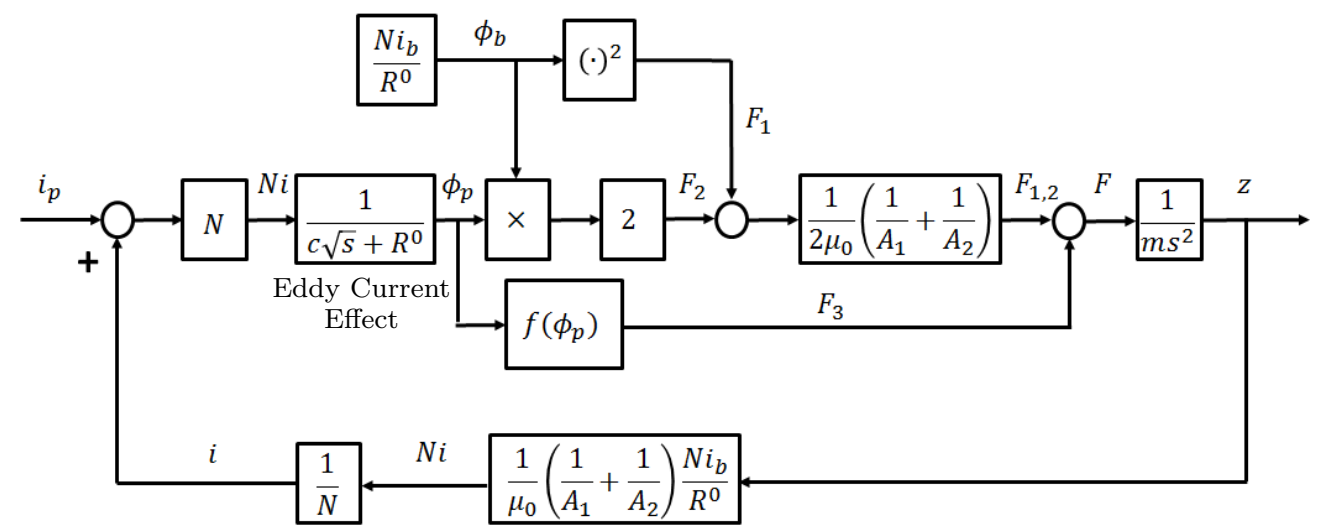

Figure 4.6: Nonlinear model with full Maxwell stress tensor approximated using average flux density.

While the relationship between perturbation flux and force given by the third term in Equation 4.2 is more complicated than that of the other force components, it may be approximated simply by integrating the average flux density over the pole surface area. For thrust AMBs with a center hole, the equation describing the distribution of flux density across the air gap at the inner pole face for a given magnetomotive force was derived in Chapter 2 and is shown again here for convenience:

$$
B_{p}(r)=\frac{\mu_{0} F}{l_{g}}\left[\frac{K_{1}\left(\alpha_{1} r_{0}\right) I_{0}\left(\alpha_{1} r\right)+I_{1}\left(\alpha_{1} r_{0}\right) K_{0}\left(\alpha_{1} r\right)}{I_{1}\left(\alpha_{1} r_{0}\right) K_{0}\left(\alpha_{1} r_{1}\right)+I_{0}\left(\alpha_{1} r_{1}\right) K_{1}\left(\alpha_{1} r_{0}\right)}\right]
$$

Flux was determined by integrating the flux density over the inner pole surface area, resulting in:

$$
\phi_{p}=\int_{r_{0}}^{r_{1}} B_{g}(r) \cdot 2 \pi r \mathrm{~d} r=\frac{2 \pi r_{1}}{\alpha_{1}} \frac{\mu_{0} F}{l_{g}}\left[\frac{I_{1}\left(\alpha_{1} r_{1}\right) K_{1}\left(\alpha_{1} r_{0}\right)-I_{1}\left(\alpha_{1} r_{0}\right) K_{1}\left(\alpha_{1} r_{1}\right)}{I_{1}\left(\alpha_{1} r_{0}\right) K_{0}\left(\alpha_{1} r_{1}\right)+I_{0}\left(\alpha_{1} r_{1}\right) K_{1}\left(\alpha_{1} r_{0}\right)}\right]
$$


Rearranging 4.5 and substituting $B_{p}(r) \frac{1}{K_{1}\left(\alpha_{1} r_{0}\right) I_{0}\left(\alpha_{1} r\right)+I_{1}\left(\alpha_{1} r_{0}\right) K_{0}\left(\alpha_{1} r\right)}$ results in the expression describing the distribution of flux density to be:

$$
B_{p}(r)=\frac{\alpha_{1} \phi_{g}}{2 \pi r_{1}} \frac{K_{1}\left(\alpha_{1} r_{0}\right) I_{0}\left(\alpha_{1} r\right)+I_{1}\left(\alpha_{1} r_{0}\right) K_{0}\left(\alpha_{1} r\right)}{I_{1}\left(\alpha_{1} r_{1}\right) K_{1}\left(\alpha_{1} r_{0}\right)-I_{1}\left(\alpha_{1} r_{0}\right) K_{1}\left(\alpha_{1} r_{1}\right)}
$$

The frequency response of the $B_{p}^{2}$ component of force $\left(F_{3}\right)$ and the total force was approximated by numerical integration over 100 discrete regions and compared to the result obtained using the average flux density. This comparison was analyzed for the worst-case scenario where the perturbation current is equal to the bias current (Figure 4.7). The perturbation force and total force are normalized to the static case determined by numerical integration. Figure 4.7 shows that there is very little difference between the two methods: a difference of $0.2 \mathrm{~dB}$ and $4 \mathrm{deg}$ at $1000 \mathrm{~Hz}$ for the $B_{p}^{2}\left(F_{3}\right)$ component of force alone. In addition, the sum of the squared perturbation flux density over the pole face represents only a small component of the total force. Plotting the total force, determined by numerical integration compared to the approximation using the average perturbation flux density, the difference can be seen to be negligible over the frequency range of interest. Therefore, in order to simplify FBL and the simulation, this approximation was incorporated into the model as shown in Figure 4.8.

Magnitude: $F_{3}\left(B_{p}^{2}\right.$ Component of Force)

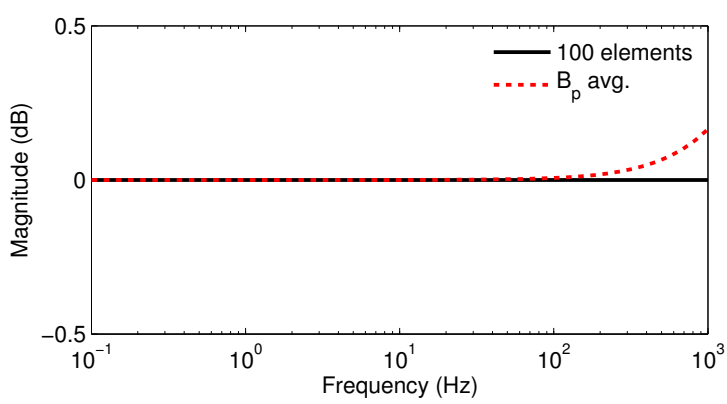

Phase: $F_{3}\left(B_{p}^{2}\right.$ Component of Force)

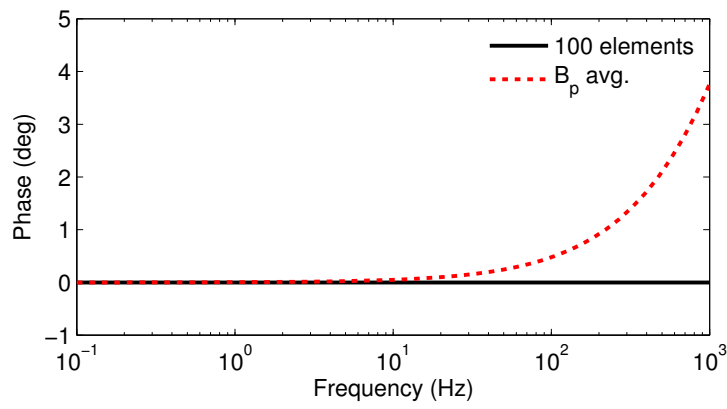

Magnitude: Total Force

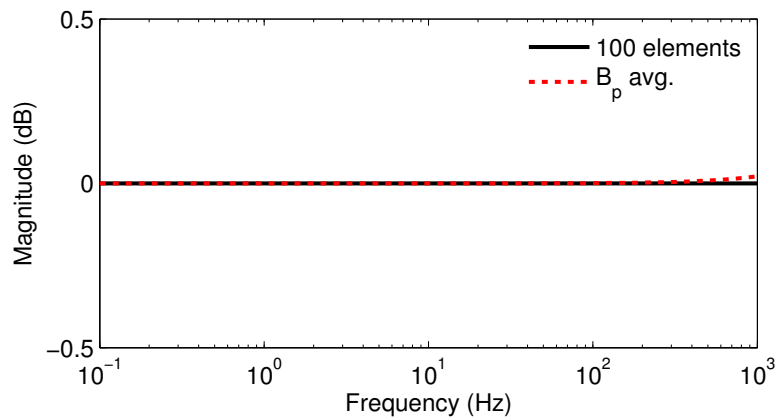

Phase: Total Force

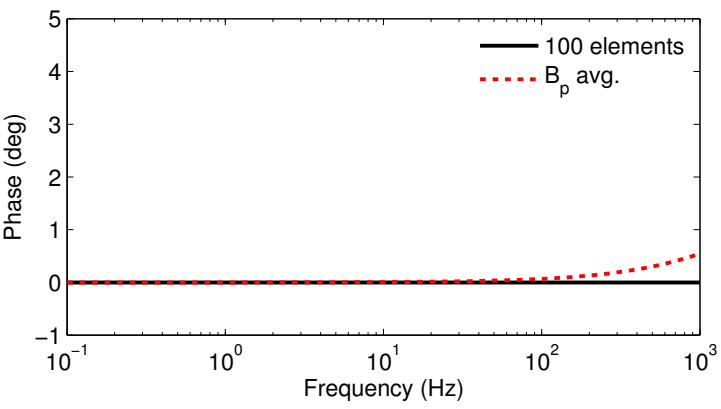

Figure 4.7: $F_{3}$ and total force, magnitude and phase, were determined by numerical integration or by an approximation using the average flux density. 


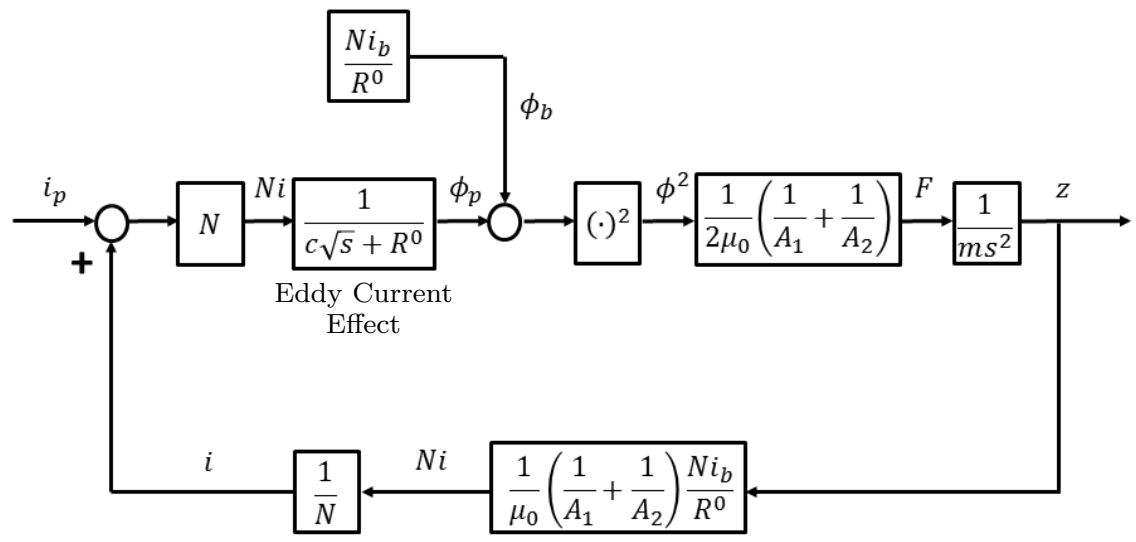

Figure 4.8: Nonlinear model with full Maxwell stress tensor.

The primary reason for using FBL is to make the force response more consistent across the range of operating positions. Typically the AMB model is linearized about the centered position. Here the position is treated as time-varying such that the linearized model changes with position. It is assumed that position $(z)$ changes slower than perturbation current $\left(i_{p}\right)$ and perturbation flux $\left(\phi_{p}\right)$ flux and is treated as quasi-steady. The static reluctance $\left(R^{0}\right)$, dependent on position $(z(t))$, then becomes a time-varying coefficient in the transfer function representing eddy current effect as shown in Figure 4.9, where $K_{f}=\frac{1}{2 \mu_{0}}\left(\frac{1}{A_{1}}+\frac{1}{A_{2}}\right)$.

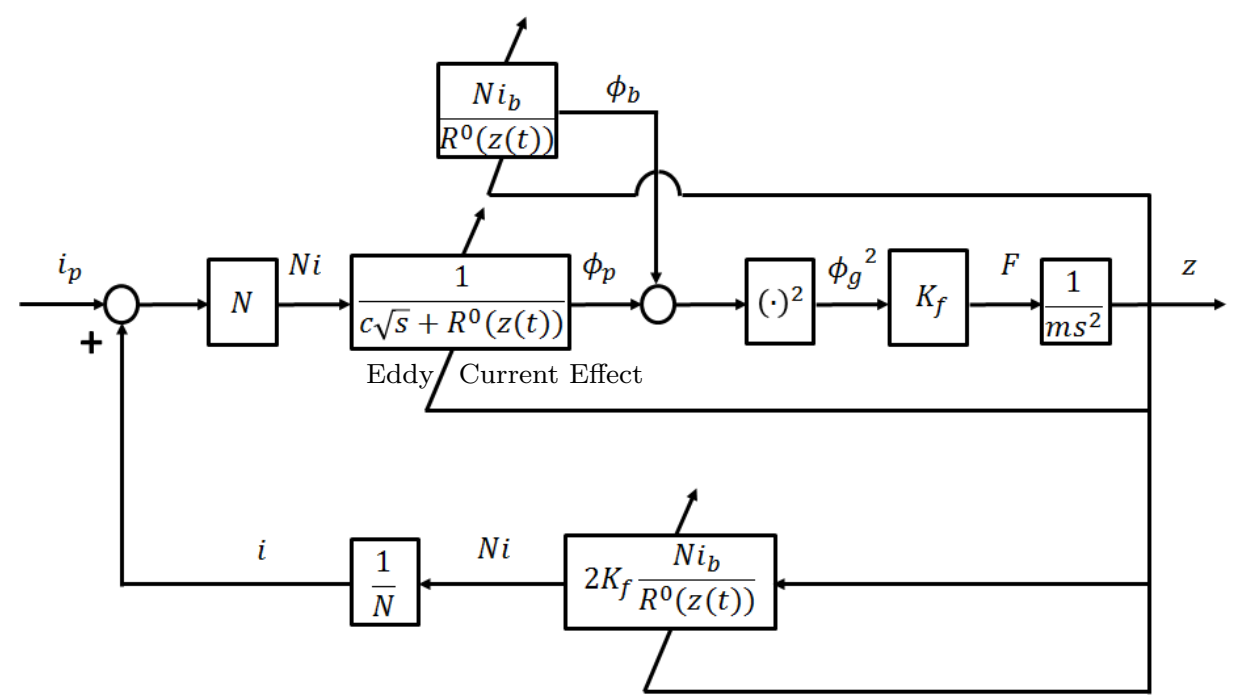

Figure 4.9: Nonlinear model with full Maxwell stress tensor approximated using average perturbation flux density.

In Figure 4.10 the bias current input has been moved to the same node as the perturbation current input. Bias current is generally static and therefore unaffected by eddy currents. However, this change simplifies development of the FBL and models the bias current step input during startup which does produce eddy 
currents for a short period of time. With these modifications the nonlinear model is complete and ready to be used for the development of a nonlinear and dynamic compensator.

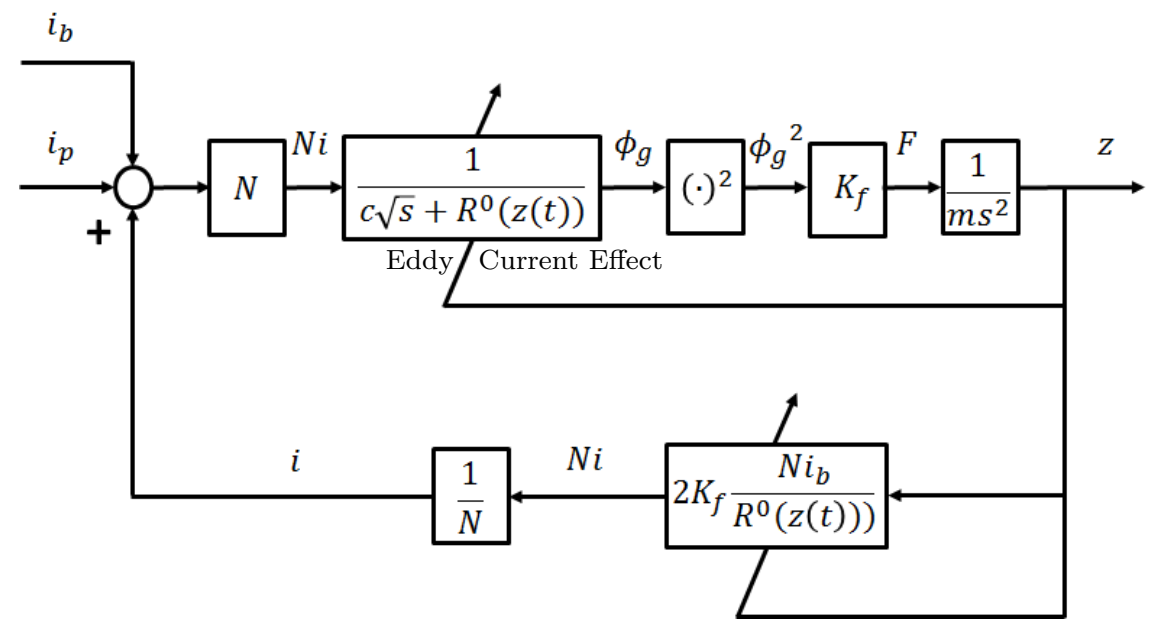

Figure 4.10: Complete nonlinear model, including eddy current effects, with with time-varying static reluctunce dependent on state.

\subsection{Feedback Linearization}

The complete model of a single sided thrust AMB may be represented more concisely as shown in Figure 4.11. The controller for the linearized system will output a desired control force $\left(F_{c}\right)$ and the goal of nonlinear and dynamic compensation is to generate an appropriate current reference signal $\left(i_{p}\right)$ in order to match the desired force $\left(F_{c}\right)$.

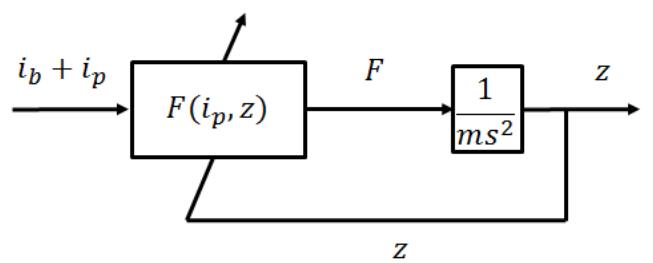

Figure 4.11: Concise nonlinear model.

$$
F\left(i_{p}, z\right)=\left[\left(\frac{N}{c \sqrt{s}+R^{0}(z)}\right)\left(i_{p}+i_{b}+2 K_{f} \frac{i_{b}}{R^{0}(z)} z\right)\right]^{2} K_{f}
$$

Solving for $i_{p}$ in Equation 4.7, with $F=F_{c}$, results in:

$$
i_{p}\left(F_{c}, z\right)=-i_{b}-2 K_{f} \frac{i_{b}}{R^{0}(z)} z+\frac{c \sqrt{s}+R^{0}(z)}{N} \sqrt{\frac{F_{c}}{K_{f}}} .
$$




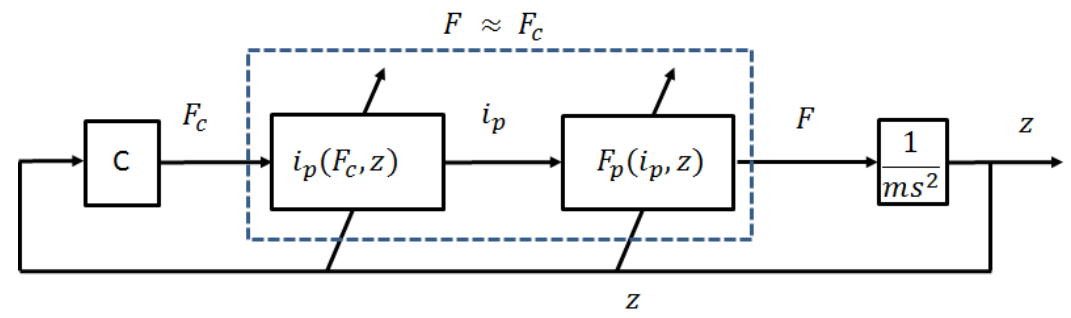

Figure 4.12: Feedback linearization for a single side of a thrust AMB.

The compensator is implemented with a bias force $\left(F_{b}\right)$ as opposed to the standard bias current formulation, such that the control force is constrained by $0<F_{c}<F_{b}$ and the perturbation current is real-valued.

With FBL complete, the entire thrust-AMB plant model becomes $\frac{1}{m s^{2}}$. As will be demonstrated in the following sections, conversion of the plant model via nonlinear compensation offers a few advantages in addition to simplifying control design. The first advantage is in dealing with large signals such as disturbance forces where nonlinear compensation improves performance consistency by inverting the nonlinear effect of position. The second advantage is improved reference tracking at low frequencies. Finally, sensitivity to external disturbance forces is also improved.

\subsection{Control Design}

To investigate potential performance advantages offered by FBL, a non-laminated thrust bearing test case is presented. The test case is based on the thrust AMB of the compressor surge test rig mentioned in previous chapters, details may be found in $[26,24,25]$. For convenience, the design parameters used here are shown in Table 4.1. PID control with FBL, referred to as FBL-PID is compared to PID control alone, referred to as PID.

The thrust bearing test case was originally designed with a high open-loop stiffness in order to support the large axial load of a compressor. With such high open-loop stiffness, the closed-loop performance achievable with standard PID was found to be insufficient. A step response to a position reference input will always have a large overshoot, more than $40 \%$, without a pre-filter. A pre-filter, $F_{f}$, was chosen to eliminate differentiation of a step change in error that would otherwise occur. With the pre-filter given by

$$
F_{f}=\frac{K p}{K d s+K p}
$$


Table 4.1: AMB Test Case Parameters

\begin{tabular}{lc} 
Parameter & Value \\
\hline$r_{0}$ & $51.7 \mathrm{~mm}$ \\
$r_{1}$ & $68.3 \mathrm{~mm}$ \\
$r_{2}$ & $90.9 \mathrm{~mm}$ \\
$r_{3}$ & $101.3 \mathrm{~mm}$ \\
$d_{1}$ & $16.5 \mathrm{~mm}$ \\
$d_{2}$ & $26.3 \mathrm{~mm}$ \\
$d_{3}$ & $15.2 \mathrm{~mm}$ \\
$l_{g}$ & $1.0 \mathrm{~mm}$ \\
$N$ & 166 \\
$u_{r}$ & 1000 \\
$\sigma$ & $2.0 \times 10^{6} \mathrm{~S} / \mathrm{m}$ \\
stator cuts & 0 \\
$i_{b}$ & $5 \mathrm{~A}$ \\
$K_{x}$ & $4.76 \times 10^{6} \mathrm{~N} / \mathrm{m}$ \\
$K_{i}$ & $995 \mathrm{~N} / \mathrm{A}$ \\
$F_{\text {max }}$ & $2488 \mathrm{~N}$ \\
AMB bandwidth & $40 \mathrm{~Hz}$ \\
\hline
\end{tabular}

Using a -pre-filter, as in Figure 4.13, derivative response to reference inputs is canceled and derivative action is taken only in response to changes in the output rather than the error. A pre-filter does not effect closed-loop response to sensor noise or a disturbance input.

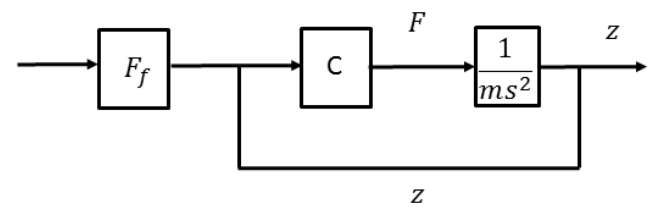

Figure 4.13: Block diagram representation of PID with pre-filter $F_{f}$.

PID parameters were obtained by exhaustive search and refined by optimization of a set of performance metrics including the minimization of peak sensitivity, overshoot, and settling times with the rise time constrained to be greater than $0.008 \mathrm{sec}$ to minimize the required force slew rate. The resulting PID gains have closed-loop response characteristics summarized in Table 4.2 .

PID parameters for the FBL-PID system were chosen to yield a closed-loop transfer function denominator equivalent to the optimized PID control design, neglecting eddy current effects. For the PID system, a simplified closed-loop transfer function neglecting eddy current effects, amplifiers, and the low pass filter in 
Table 4.2: Closed Loop Performance

\begin{tabular}{lcc} 
Parameter & PID \\
\hline Peak Sensitivity & Step Response $(0.1 \mathrm{~mm})$ & 2.76 \\
\hline & & $21 \%$ \\
Overshoot & & $0.008 \mathrm{sec}$ \\
Rise Time & $0.22 \mathrm{sec}$ \\
Settling Time & Disturbance Response $(1000 \mathrm{~N})$ & \\
\hline & & $4.7 \times 10^{-5} \mathrm{~mm}$ \\
Peak & & $0.31 \mathrm{sec}$ \\
Settling Time &
\end{tabular}

the controller is given by

$$
G_{P I D}=\frac{K_{i} K_{D} s^{2}+K_{i} K_{P} s+K_{i} K_{I}}{m s^{3}+K_{i} K_{D} s^{2}+\left(K_{i} K_{P}-2 K_{x}\right) s+K_{i} K_{I}} .
$$

For the FBL-PID system, a simplified closed-loop transfer function neglecting amplifiers, and the low pass filter in the controller is given by

$$
G_{F B L-P I D}=\frac{K_{D} s^{2}+K_{P} s+K_{I}}{m s^{3}+K_{D} s^{2}+K_{P} s+K_{I}}
$$

Equating the denominator coefficients and solving for the PID parameters in the FBL-PID system we arrive at the following parameter values for FBL-PID control:

$$
\begin{aligned}
& K_{P(F B L-P I D)}=K_{i} K_{P(P I D)}-K_{x}, \\
& K_{I(F B L-P I D)}=K_{i} K_{I(P I D)}, \text { and } \\
& K_{D(F B L-P I D)}=K_{i} K_{D(P I D)} .
\end{aligned}
$$

With equivalent closed-loop systems, any differences in performance may be attributed to the FBL rather than the PID component of the control design. The result is two systems with very similar nominal closedloop performance. However, there are some differences that suggest FBL may be advantageous for certain applications. In the next section a linear analysis is performed comparing PID with FBL-PID.

\subsection{Linear Performance Analysis}

Linear analysis presented in this section provides a general idea of how each system will perform for small signal inputs and assuming that the plant model is a perfect representation of the physical plant. The benefits 
of nonlinear compensation for large signals will not appear in a linear analysis. However, several advantages of FBL are seen by linear analysis which are due to dynamic compensation included in FBL. It is clear from the step response (Figure 4.14) that while control by PID alone results in $20 \%$ overshoot, there is no overshoot with control by FBL-PID. Normally there is a trade-off between overshoot in response to a step reference input and peak response due to a step disturbance. However, FBL-PID enables improvements in both reference tracking and disturbance rejection (Figure 4.14) where FBL-PID has a peak response to a step disturbance input that is reduced by $17 \%$ while the disturbance peak is also reduced by $20 \%$ compared to PID alone.

Sensitivity and complimentary sensitivity are probably the two most highly referenced transfer functions in control design. The sensitivity function $(S(s))$ represents the sensitivity of the system to small variations in the plant [27]. The sensitivity function also represents the system transfer function from reference input to tracking error, which means low sensitivity gains are desired over the relevant frequency range of the reference input. Peak sensitivity $\left(S_{M}\right)$ is often used as a measure of robustness, analogous to phase or gain margin. According to the ISO 14839-2 specification for performance of AMB systems, newly commissioned machines should have a peak sensitivity below $9.5 \mathrm{~dB}[28]$. The complimentary sensitivity function $(T(s))$ is simply the closed loop transfer function but is referred to as complimentary sensitivity because $S(s)+T(s)=1$.

Peak sensitivity is similar for PID and FBL-PID, with $M_{S(P I D)}=7.61 \mathrm{~dB}$ and $M_{S(F B L-P I D)}=7.80 \mathrm{~dB}$, both well below the recommended limit suggesting similar robustness for both systems (Figure 4.15. The sensitivity function for FBL-PID compared to PID has a lower magnitude in the low frequency region, suggesting that FBL-PID will be less sensitive to small variations in the physical plant and will perform better than PID alone in reference tracking (Figure 4.15). The peak sensitivity is also shifted to a higher frequency for FBL-PID, as is the sharp decrease in phase which is seen in both systems. These results suggest that the reference tracking performance of FBL-PID will have less phase lag than PID alone.

The complimentary sensitivity function $(T)$, or closed-loop transfer function, also demonstrates some improvements for FBL-PID compared to PID alone. The magnitude of the complimentary sensitivity function for PID alone is just above $0 \mathrm{~dB}$ at low frequency, suggesting that there will be some amplification in tracking low frequency reference inputs (Figure 4.16). For FBL-PID, the magnitude of $T$ is closer to $0 \mathrm{~dB}$ (Figure 4.16). This is consistent with the step response (Figure 4.14) showing $20 \%$ overshoot for PID with no overshot for FBL-PID. Similar overshoot is expected for reference inputs below $10 \mathrm{~Hz}$. For FBL-PID, the magnitude of $T$ is slightly lower than PID alone over the frequency range from 20 to $50 \mathrm{~Hz}$, suggesting that for reference inputs in this frequency range, the magnitude of a reference input will be matched better by PID alone. However, for FBL-PID the phase of $T$ is extended to higher frequencies (Figure 4.16) suggesting a phase improvement in reference tracking for FBL-PID compared to PID alone for higher frequency reference signals. 
FBL also results in a simpler linear plant model. As a result, the complimentary sensitivity function has a flatter magnitude at low frequencies whereas for the PID system the complimentary sensitivity gain creeps slightly above 1.0 before rolling off. This flatter magnitude should result in better tracking of low frequency reference inputs for FBL-PID. Using PID control there is a trade-off between overshoot and peak disturbance response. However, FBL seems to improve this relationship.

Sinusoidal reference tracking is not generally a required feature of magnetic bearing control. However, as shown by Yoon et al. [24, 25], suppression of compressor surge can be accomplished using a surge controller to track a desired axial impeller position. By controlling the impeller tip clearance, a pressure wave can be generated to cancel surge-induced oscillations. Yoon et al. used $H_{\infty}$ control to track axial rotor position. Based on the above discussion, FBL-PID may offer a simple alternative to this solution. The complimentary sensitivity function suggests that FBL-PID will track a low frequency position reference well, but will not perform as well tracking reference frequencies above $20 \mathrm{~Hz}$. Compressor surge is typically a low frequency disturbance, often close to $5 \mathrm{~Hz}$. Therefore, in order to cancel surge oscillations, reference tracking at low frequencies may be sufficient.

Another relevant performance related transfer function is the closed loop response to disturbance force inputs. This is useful when the AMB is to reject disturbance forces associated with surge and stall events in compressors, or cutting forces in machining. The frequency response to disturbance forces (Figure 4.17) shows another advantage of FBL-PID. At low frequencies both systems are predicted to behave similarly in response to disturbance forces. However, as the frequency increases, the magnitude for FBL-PID in response to a disturbance is significantly lower than for PID alone in the frequency range from 100 to $400 \mathrm{~Hz}$. This may not be an advantage in rejecting low frequency disturbance forces resulting from compressor surge. However, this analysis predicts an advantage for control by FBL-PID in rejecting higher frequency disturbance forces, such as those associated with compressor stall and emergency trip events or high frequency vibrations frequently encountered in machining.

The loop transfer function provides an analysis of robustness analogous to peak sensitivity. Gain margin (GM) represents the increase in gain that would drive the system unstable and phase margin represents the increase in phase lag required to drive the system unstable. GM is given by

$$
G M=\frac{1}{\left|L\left(j \omega_{g}\right)\right|},
$$

where $\omega_{g}$ is the phase crossover frequency of $(-180 \mathrm{deg})$. Phase margin $(\mathrm{PM})$ is given by

$$
P M=\pi+\operatorname{Arg}\left(L\left(j \omega_{c}\right)\right)
$$


where $\omega_{c}$ is the crossover frequency where the magnitude of $L$ crosses $0 \mathrm{~dB}$. GM and PM values for PID and FBL-PID are shown in Table 4.3 along with the peak sensitivity for reference. The GM and PM for PID and FBL-PID are similar for the two systems. This is not surprising since these two measures of robustness determine the peak sensitivity $\left(M_{S}\right)$ which is the shortest distance from the Nyquist curve to the critical point at -1 . The loop gain frequency response (Figure 4.18) also shows that FBL is successful in extending the bandwidth. With PID alone, the loop gain crosses $-3 \mathrm{~dB}$ at $399 \mathrm{~Hz}$ whereas for FBL-PID, the loop gain crosses $-3 \mathrm{~dB}$ at $811 \mathrm{~Hz}$. This result shows that in this case the frequency range over which the controller is effective is approximately doubled.

Table 4.3: Linear Robustness Parameters

\begin{tabular}{lcc} 
& PID & FBL-PID \\
\hline$M_{S}$ & $7.6 \mathrm{~dB}$ & $7.8 \mathrm{~dB}$ \\
$G M$ & $9.5 \mathrm{~dB}$ & $7.6 \mathrm{~dB}$ \\
$P M$ & $29 \mathrm{deg}$ & $31 \mathrm{deg}$ \\
\hline
\end{tabular}

Using equivalent controllers, linear analysis predicts that FBL-PID control will result in improved performance of low frequency reference tracking and high frequency disturbance rejection while degrading performance of high frequency reference tracking. However, performance tracking for a high frequency reference input is generally not a significant concern for AMB systems.

Linear analysis assumes perfect nonlinear compensation and operation at the nominal position for PID control. However, not all system nonlinearities are included in the FBL compensation formulation. Leakage and fringing are neglected as is flux saturation. Modeling errors will also affect FBL-PID performance. However, FBL-PID may improve performance by compensating for some of the nonlinearities and improving consistency over the operating range. Using the detailed nonlinear model developed in Section 4.2, Simulink models were created to study system performance of both standard PID and FBL-PID control. In the next section nonlinear analysis of each control method will be compared.

\subsection{Nonlinear Performance Analysis}

One of the most striking advantages of FBL is an improved performance consistency across the entire air gap. Consistent performance over the operating range has been mentioned previously as an advantage of FBL. Using a detailed nonlinear Simulink model, reference tracking performance across the air gap is compared for PID control alone and FBL-PID control methods (Figure 4.19). Beginning with a centered rotor, a series of $0.1 \mathrm{~mm}$ reference step inputs were tracked by each controller. As shown by linear analysis (Section 4.5), 
PID alone results in significant overshoot compared to FBL-PID. As the rotor moves farther from center, the overshoot with PID alone increases. During the step from 0.4 to $0.5 \mathrm{~mm}$, the system controlled by PID becomes unstable, while FBL-PID control results in consistent step response tracking across the entire air gap. This simulation demonstrates the performance consistency resulting from nonlinear compensation which cannot be captured by linear analysis.

Simulation results also demonstrate that tracking a sinusoidal reference input can be be improved by FBL-PID for reference inputs at frequencies below $10 \mathrm{~Hz}$, especially for large amplitude reference inputs (Figure 4.20). However, sinusoidal reference tracking performance of FBL-PID degrades as the frequency increases beyond $10 \mathrm{~Hz}$. With a reference input at $50 \mathrm{~Hz}$, both control methods have a similar phase lag but the magnitude resulting from FBL-PID is lower than for PID alone. The reduced magnitude of the FBL system compared to standard PID is consistent with the frequency response shown in Figure 4.15.

While tracking performance for high frequency reference inputs is degraded by FBL-PID, rejection of higher frequency disturbance inputs is predicted to improve. Figure 4.21 shows the response to sinusoidal disturbance forces with an amplitude $1000 \mathrm{~N}$ and frequencies of 5 and $50 \mathrm{~Hz}$. In each case, FBL-PID improves the disturbance rejection capability compared to PID alone, with greater improvement in response to the higher frequency disturbance forces. This result is consistent with linear analysis (Figure 4.17), which predicts that FBL-PID will improve disturbance rejection of sinusoidal forces with frequencies up to $800 \mathrm{~Hz}$.

Rejection of step force disturbance inputs is also improved by FBL-PID as shown in Figure 4.23. This result is consistent with linear analysis of the disturbance response (Figure 4.17). A concern which is often raised in connection with FBL, is the possibility of increased sensitivity to noise. In order to test the effect of sensor noise, experimentally obtained sensor noise was added to the position output of the simulation. The noise signal (Figure 4.23, top)was recorded from an eddy current position sensor and was filtered using a sixth-order low-pass elliptic filter with a cutoff frequency of $6000 \mathrm{~Hz}$ prior to adding to the position signal in the simulation. Locating axial position sensors far from amplifiers and other noisy signals is normally not a problem so the amplifiers were not active during the signal acquisition. With this noise added to the position output, the simulation predicts that low amplitude noise will not be a problem for FBL-PID.

The noise signal, shown in Figure 4.24, top, has a standard deviation of $71.2 \mathrm{~nm}$. To determine the effect of higher amplitude noise, a series of increasing gains were applied to the noise signal. When the raw amplitude reached a standard deviation of $1.42 \mu \mathrm{m}$ the effect of noise was apparent in both systems but was more detrimental to FBL-PID control compared to PID alone (Figure 4.24). These results suggest that noise should be considered when implementing this type of FBL but that noise will not be problematic if noise amplitudes are minimized sufficiently. 
As shown in Figure 4.19, an impressive feature of FBL-PID is consistent performance over the entire operating range. There are several examples of AMB supported rotors which operate over a range of positions. In AMB supported compressors with large axial static loads, the operating position can be biased in opposition to the load to decrease the air gap and current requirement on the loaded stator [29]. In certain machining tasks such as drilling an oblate bore, it is necessary to operate the cutting tool off-center. In apllications like these, control by FBL-PID may be well-suited as demonstrated by the results in Figures 4.25 and 4.26 .

Figure 4.25 simulates a rotor tracking a position reference that first brings it off-center. A $1000 \mathrm{~N}$ step disturbance is then applied to simulate a compressor load. After the transient period, a decaying sinusoidal disturbance with a $1000 \mathrm{~N}$ amplitude is applied to simulate a surge event. While operating off-center, the advantage of FBL-PID in rejecting a step disturbance is more apparent than when operating in a centered position. The sinusoidal disturbance response also demonstrates the consistent performance of FBL-PID. Figure 4.26 demonstrates the performance of FBL-PID control in tracking a sinusoidal position reference while off-center. PID-FBL performs as it did in tracking this signal about the center position. However, PID control exhibits increased overshoot when tracking a sinusoidal reference about a bias position.

\subsection{Analysis of Robustness}

The analysis presented in the previous section is based on the assumption that the model used for FBL-PID is a perfect representation of the plant. In reality this is never the case. Full analytic models for non-laminated thrust AMBs are accurate and are useful for control design but may not be accurate enough for FBL. Analytic models do not include flux saturation, leakage, fringing, or hysteresis. In addition, the full analytic model developed in Chapter 2 is a cumbersome transcendental function which is not useful for FBL. The approximate analytic model, used for FBL-PID design, is a more useful transfer function model, but it also has reduced fidelity. In this section, Monte Carlo analysis is used to evaluate the robustness of control via FBL-PID with respect to model discrepancies. Random variation in the parameters of the plant model were made without any change in the model used for FBL design.

Four parameters were selected for random variation: $K_{i}, K_{x}, c$, and $R^{0}$. With a good analytic or finite element model, or experimental testing, it is reasonable for $K_{i}$ and $K_{x}$ to be accurate within $10 \%$. Therefore, $K_{i}$ and $K_{x}$ were varied with a uniform distribution $\pm 10 \%$ about the analytically determined parameters. To determine appropriate variations for $c$ and $R^{0}$, the frequency response of current to flux for the approximate analytic model given by

$$
\phi_{p}(s)=\frac{N}{c \sqrt{s}+R^{0}} i_{p}(s),
$$


was compared to the finite element model, including flux leakage and fringing, created using FEMM [23]. Frequency responses for each are shown in Figure 4.27, where it is clear that the approximate analytic model is not a perfect representation of the perturbation current to force relationship. $c$ and $R^{0}$ parameters were then optimized to fit the finite element model frequency response. As can be seen in Figure 4.27, the frequency response of the optimized analytic model closely matches the finite element model in both magnitude and phase. The optimized parameters differ by $\pm 4.5 \%$ and $\pm 13 \%$ for $c$ and $R^{0}$, respectively. Based on this analysis, these same deviations were used to vary the parameters $c$ and $R^{0}$ using a uniform distribution for the Monte Carlo analysis.

Response to a $1000 \mathrm{~N}$ step input was simulated for 1000 combinations of random variation in the plant parameters $K_{i}, K_{x}, c$, and $R^{0}$. The upper and lower bounds for all simulations were plotted to show disturbance rejection for best- and worst-case modeling errors. In both cases, FBL-PID results in slightly better disturbance rejection compared to PID alone. The upper and lower bounds show the same proportional advantage, around $17 \%$, for FBL-PID as seen for the case without modeling discrepancies (Figure 4.28, nominal results). While FBL may in general be susceptible to poor robustness, Monte Carlo analysis presented here suggests that in this case FBL does not impair robustness relative to PID control alone.

In addition to the Monte Carlo analysis comparing PID with FBL-PID, an idealized thrust bearing without eddy current effects was analyzed. This plant model has a static reluctance determined by $R^{0}$ but does not include losses associated with eddy currents $(c=0)$, thereby resulting in an actuator with infinite bandwidth. Disturbance rejection for this idealized thrust bearing with FBL and PID control was also compared to FBL-PID with eddy currents to determine the effectiveness of dynamic compensation. In this case, FBL compensated for nonlinearities with respect to position, but dynamic compensation of eddy current effects was unnecessary since they are absent. Results of 1000 simulations of disturbance response for the idealized plant model are nearly identical to the model with eddy current effects (Figure 4.29), suggesting that the dynamic compensation for eddy current effects was quite successful.

\subsection{Summary}

While FBL has been applied previously to AMB control, dynamic compensation for eddy current effects by FBL has not been reported in the literature. The strategy involves a simple inversion of the eddy current effects in addition to the nonlinear position effect. At low frequency the PID and FBL-PID controllers have similar gain. The effect of dynamic compensation can be seen in the frequency response of the FBL-PID control compared to PID alone (Figure 4.30). Inversion of the roll-off in the actuator magnitude response, due to eddy current effects, appears as an increase in FBL-PID control gain with increasing frequency. The 
analysis presented above suggests that FBL is a useful tool for improving performance of certain machines with non-laminated AMBs. FBL was demonstrated to improve consistency and improve the balance between reference tracking and disturbance rejection, without any loss in robustness. FBL-PID is not necessarily beneficial for all AMB applications. However, for specialty applications where a range of operating conditions are encountered, FBL with dynamic and nonlinear compensation is a promising control strategy.

The test case presented here may not represent the full potential of FBL in improving disturbance rejection. FBL has been suggested previously for use in AMBs with large air gaps. With a potentially wide operating range, performance consistency may be critical. The test case presented here does have a relatively large air gap of $1 \mathrm{~mm}$. However, with a large air gap, large displacements due to a disturbance force may not significantly change the position in the air gap enough to affect performance. With a smaller air gap displacements due to disturbance may result in a more significant change in operating conditions, in which case FBL could provide even more benefit.

In the simulations presented here, peak slew rates beyond the capability of a typical amplifier were observed. Simulations with slew rate limiting imposed demonstrated similar results for a moderate controller. However, with more aggressive controllers with fast rise times, FBL-PID was more sensitive to slew rate limits compared to PID. When implementing FBL-PID, it will be important to match control effort and hardware capabilities to alleviate these types of issues.

Another nonlinear effect to be considered is flux density saturation in the material. Because the analytic model used for FBL assumes the magnetic material is linear and isotropic, care should be taken to avoid, or compensate for, nonlinear relationships between field strength and flux density. The material used in the test case is based on low carbon steel with a saturation flux density around 1.3 T. The worst-case scenario regarding flux density saturation is encountered for high frequency perturbation currents with an amplitude equal to the bias current. Figure 4.31 shows the flux density estimation for the test case presented here with a static $5 \mathrm{~A}$ current and a $5 \mathrm{~A}$ perturbation current at $20 \mathrm{~Hz}$. Bias current results in a uniform flux distribution that does not approach saturation. However, with a $5 \mathrm{~A}$ perturbation current at $20 \mathrm{~Hz}$, flux density saturation can be observed around the coil groove. Matching hardware and control design capabilities will be important for avoiding flux saturation in order to optimize the performance of FBL-PID controlled systems. 


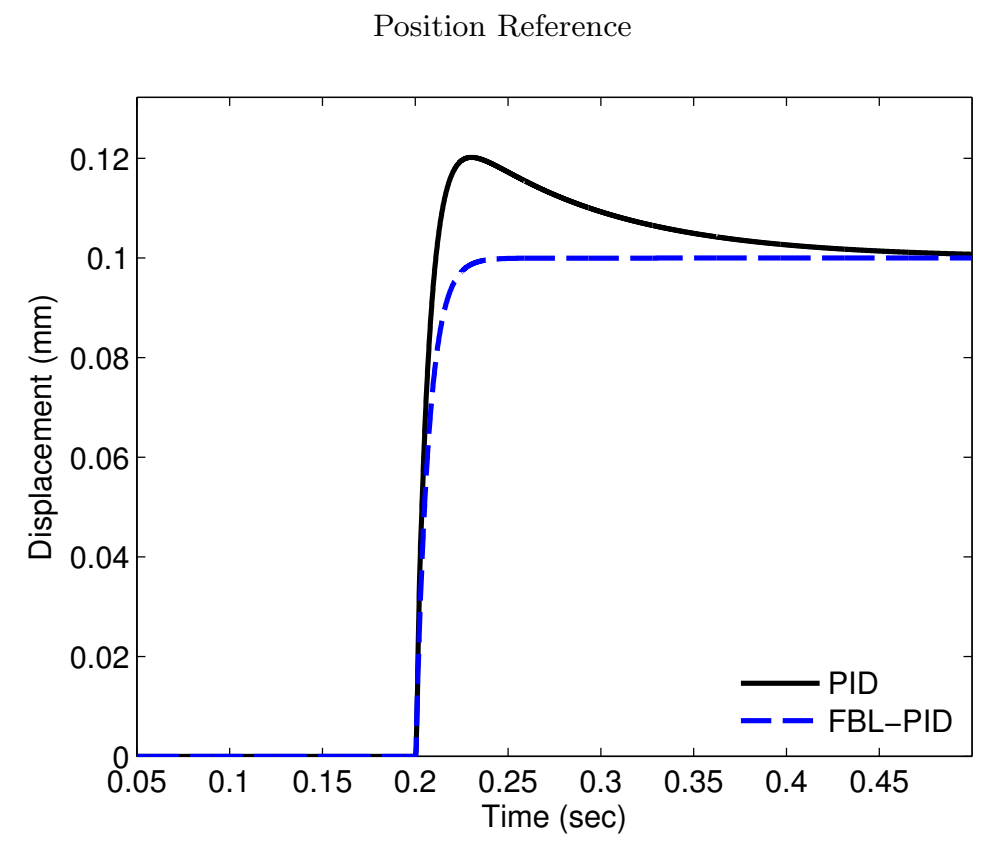

Disturbance Force

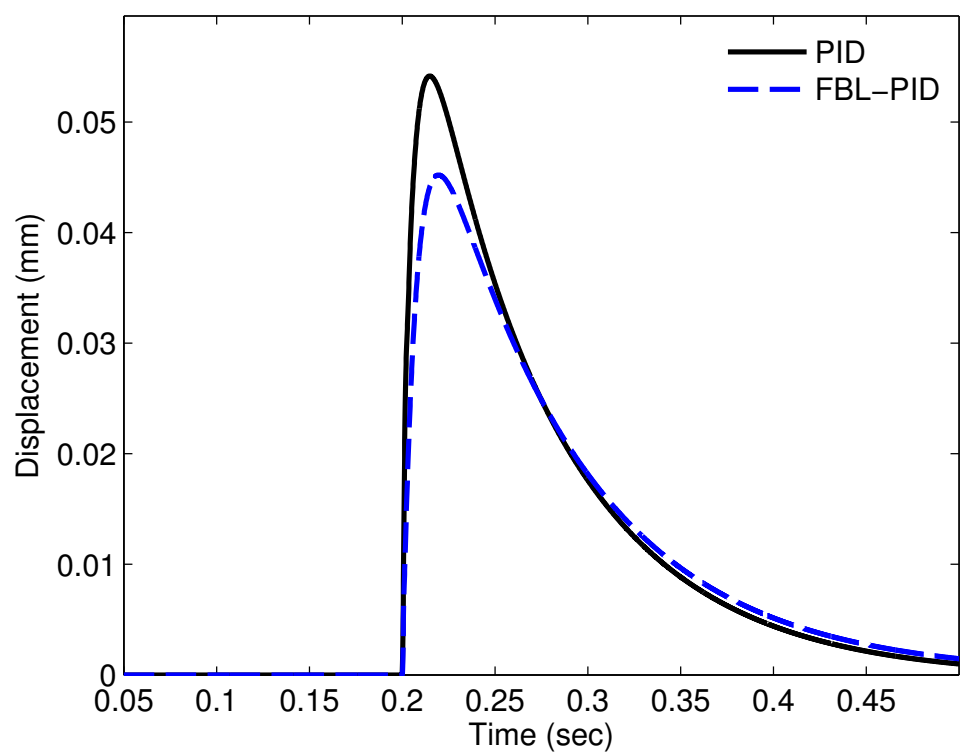

Figure 4.14: Step responses for reference and disturbance inputs $(1000 \mathrm{~N})$ based on linear analysis. 

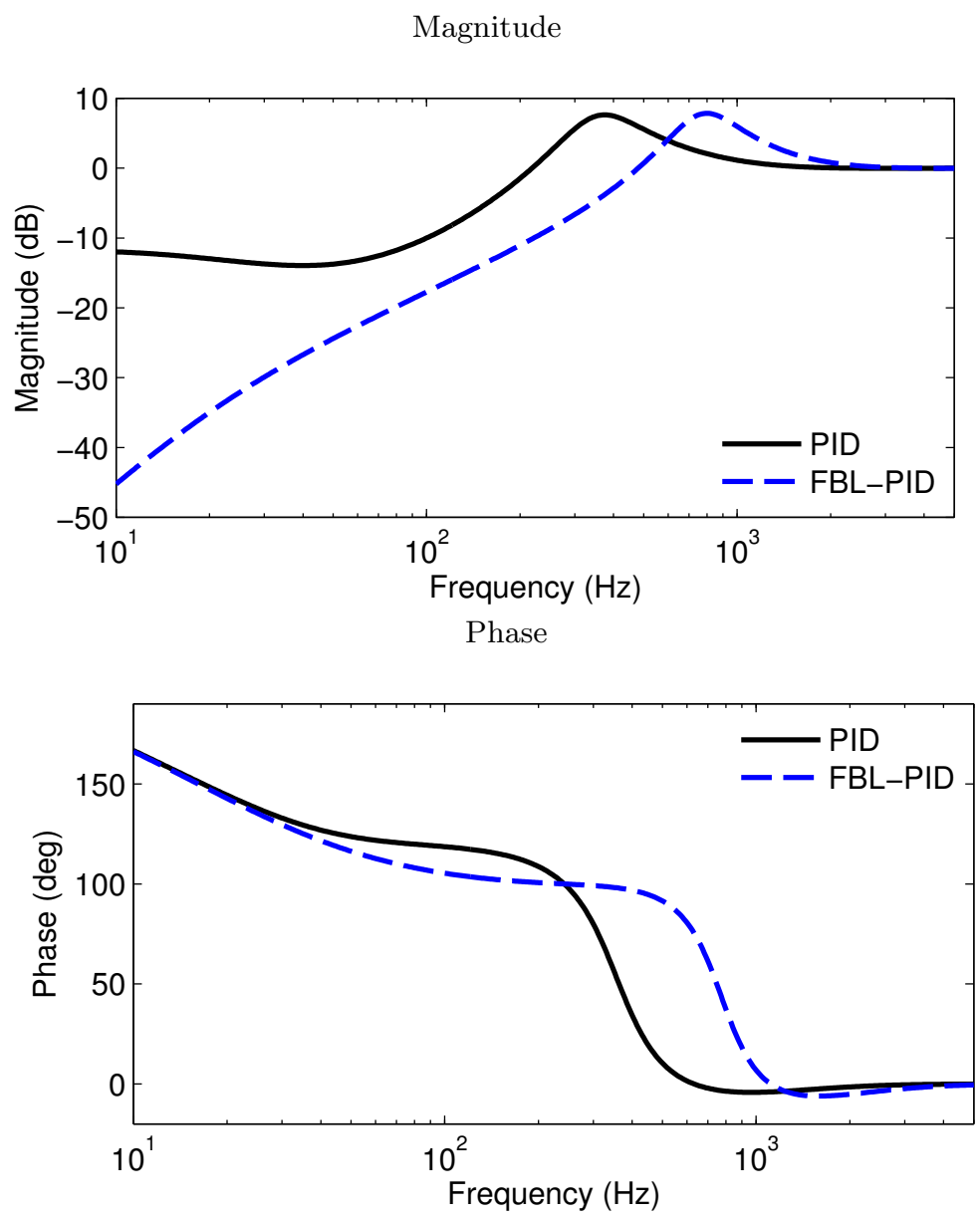

Figure 4.15: Sensitivity frequency response (Sensitivity DC gain is 0 for both systems) 

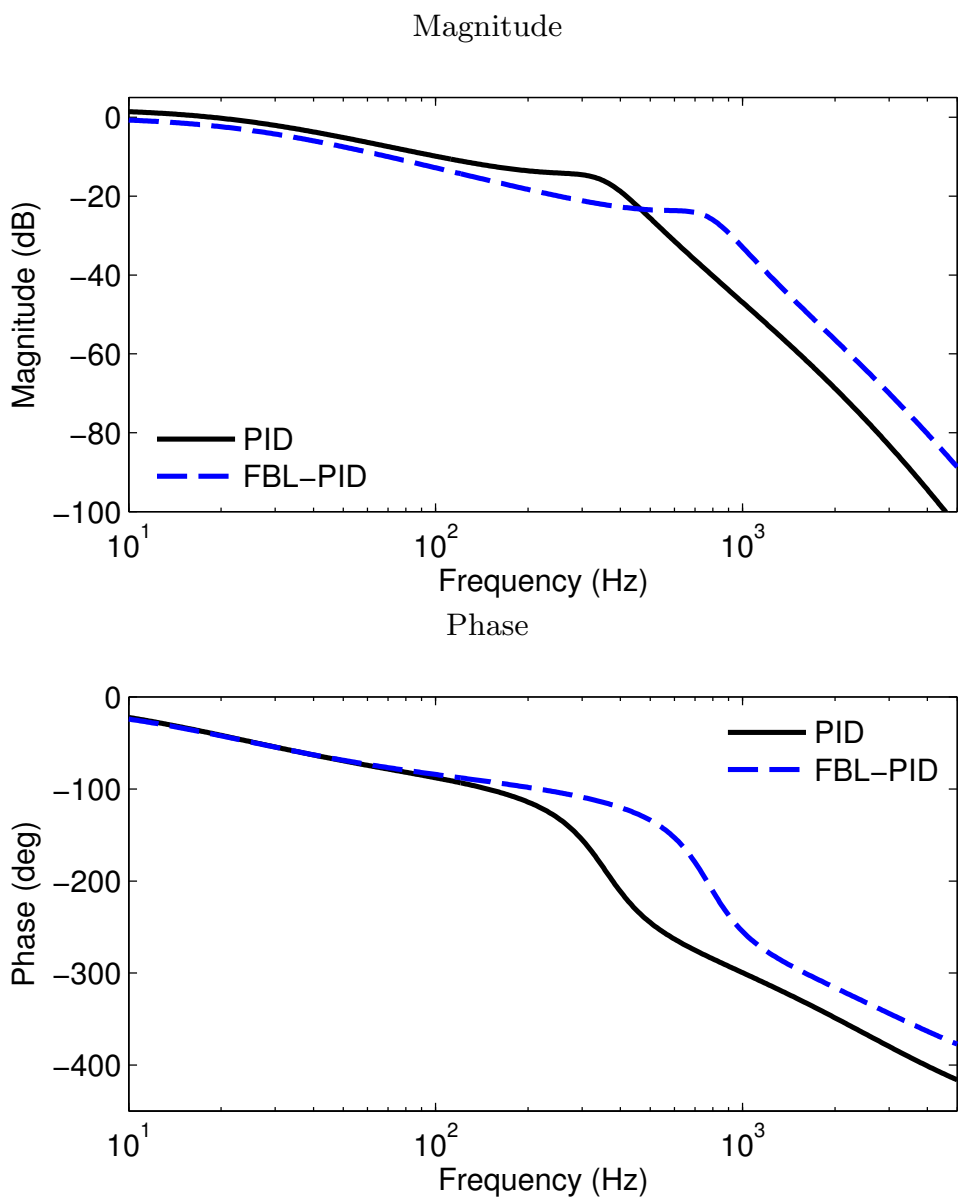

Figure 4.16: Complimentary sensitivity frequency response 

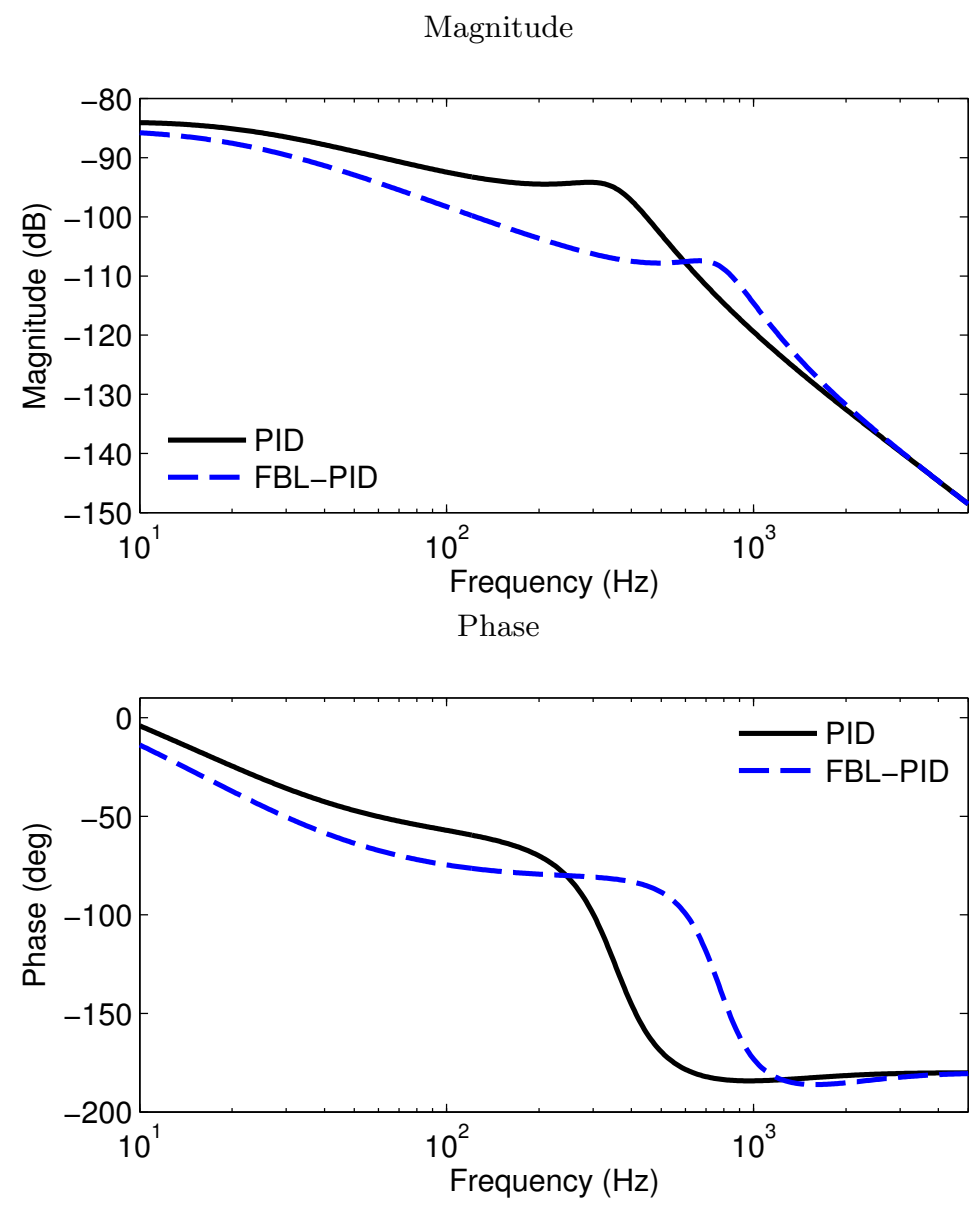

Figure 4.17: Frequency response for disturbance input $\left(\frac{m m}{N}\right)$ 

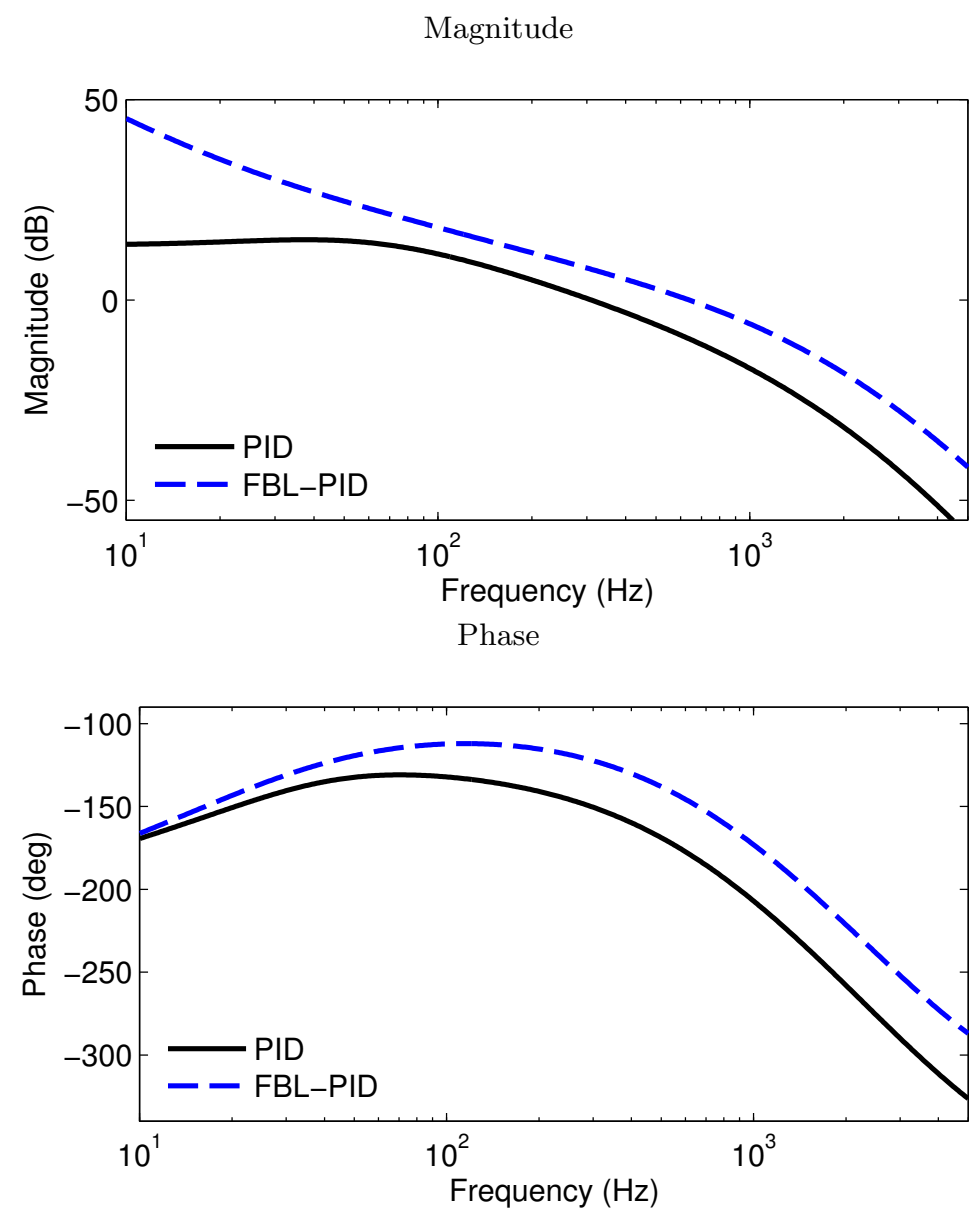

Figure 4.18: Loop gain frequency response (DC loop gain is infinite for both systems)

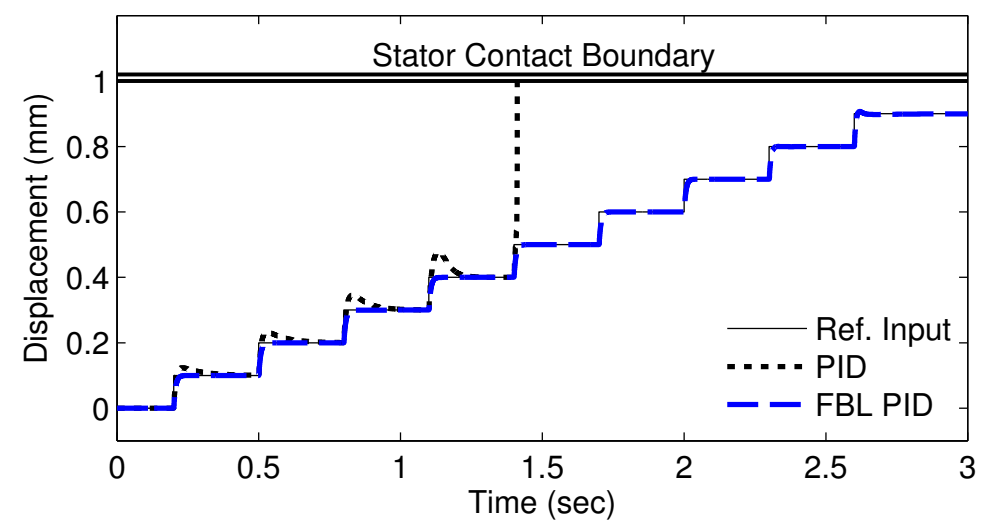

Figure 4.19: Simulated reference tracking performance for a series of step inputs. Solid lines at $1 \mathrm{~mm}$ represent the boundary where the thrust disk contacts the stator. 
Small Amplitude, $5 \mathrm{~Hz}$

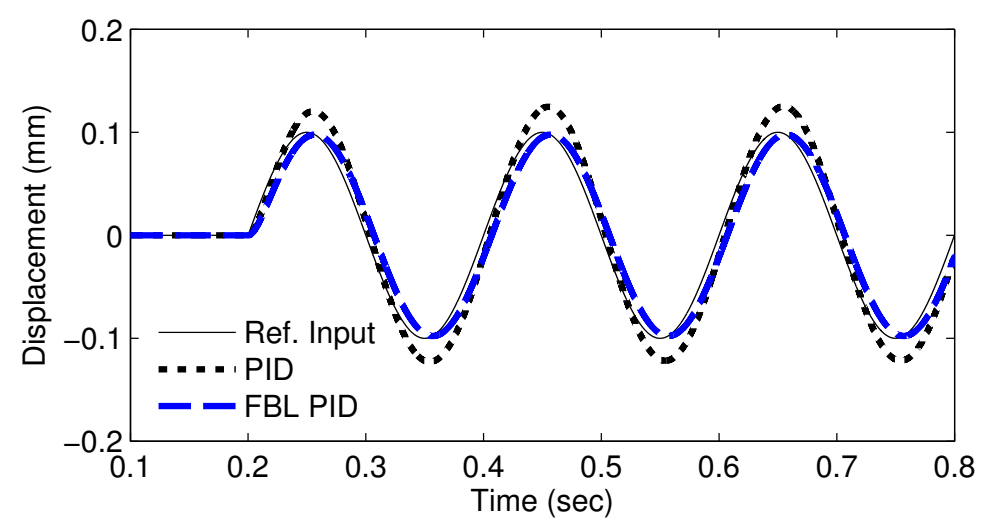

Large Amplitude, $5 \mathrm{~Hz}$

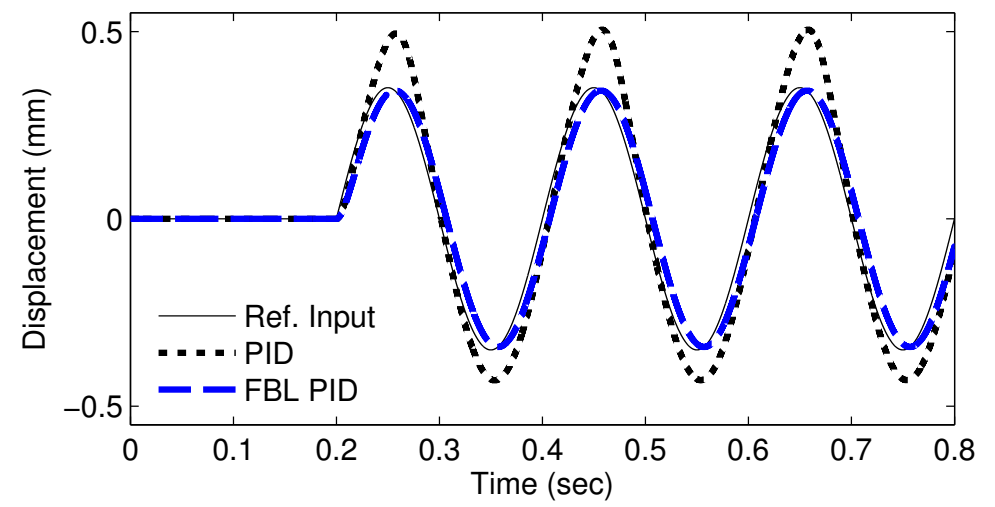

Small Amplitude, $50 \mathrm{~Hz}$

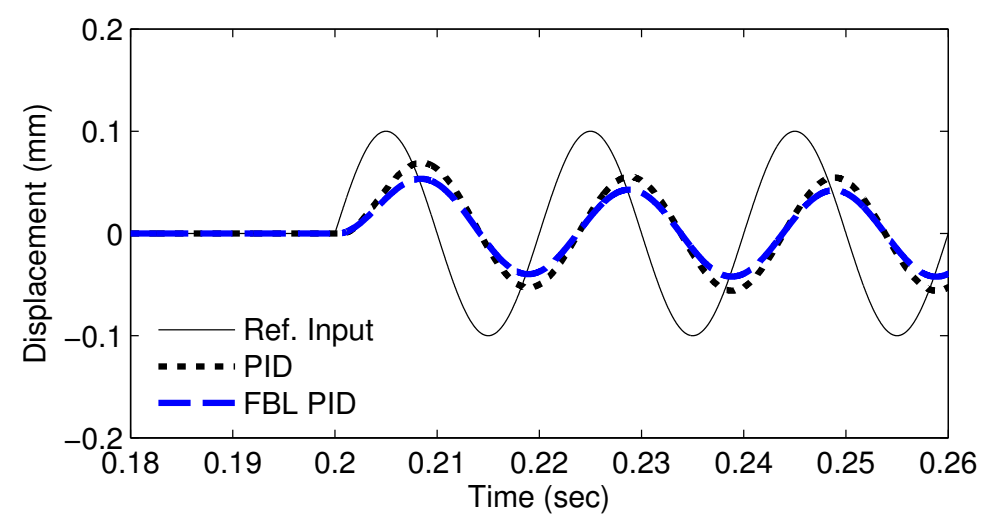

Figure 4.20: Simulated sinusoidal reference tracking with input frequencies of 5 and $50 \mathrm{~Hz}$, small and large amplitude. 
$5 \mathrm{~Hz}$
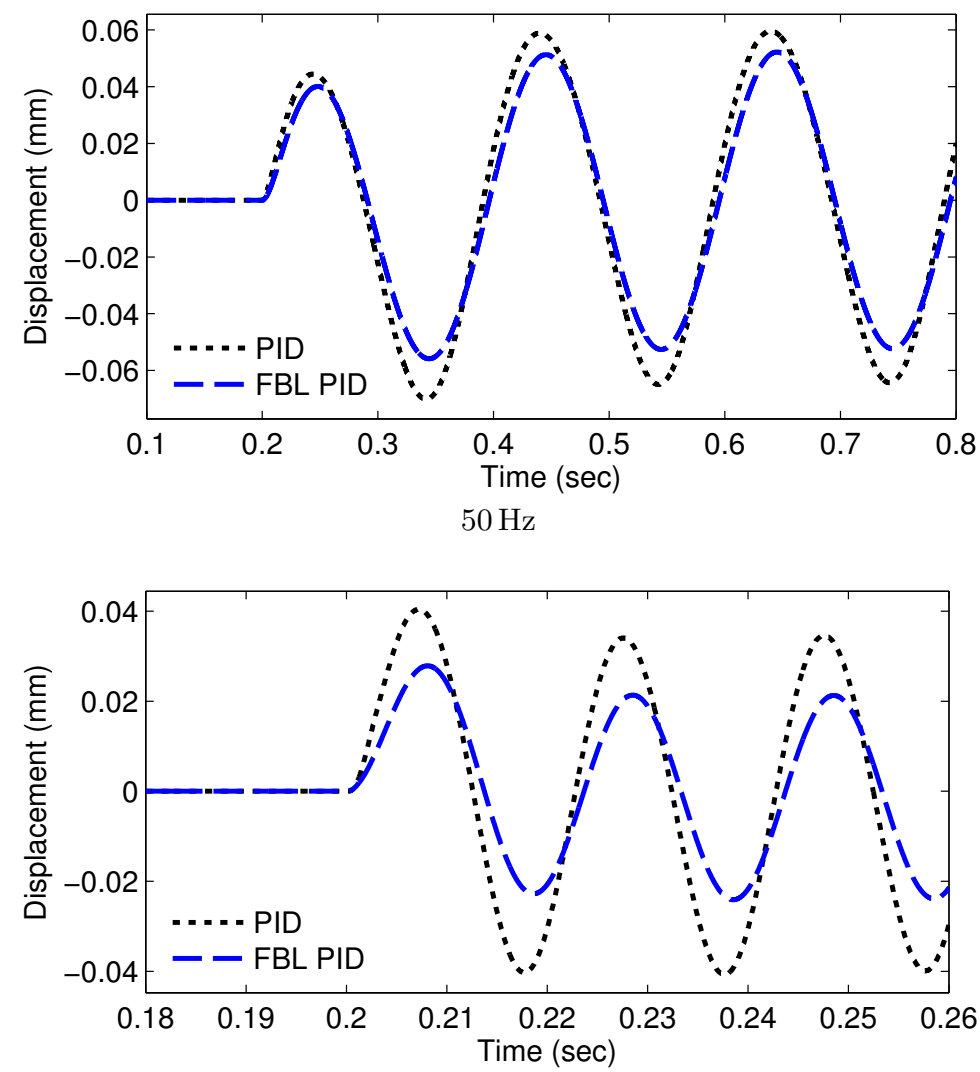

Figure 4.21: Simulated response to sinusoidal disturbance forces with frequencies of 5 and $50 \mathrm{~Hz}$.

Disturbance Rejection

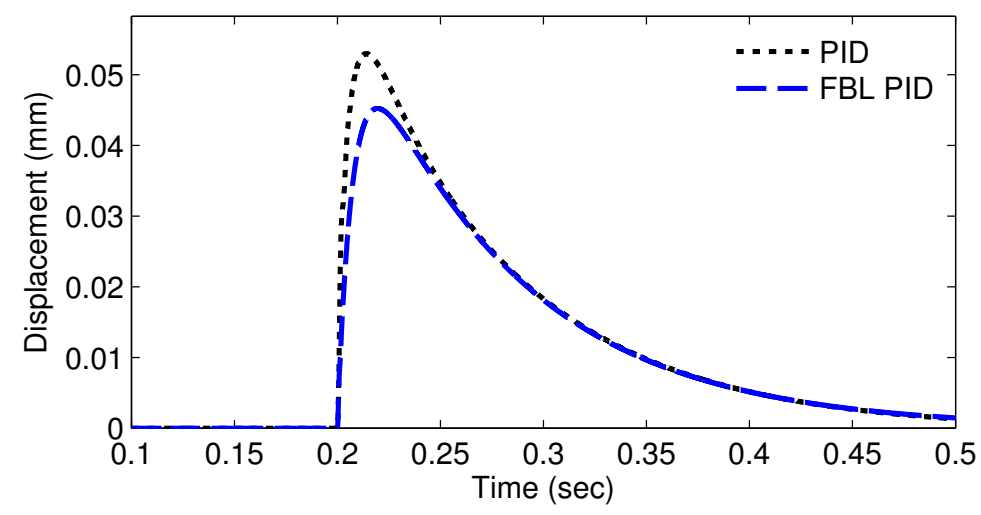

Figure 4.22: Simulated response to $1000 \mathrm{~N}$ disturbance step input. 
Unfiltered Noise
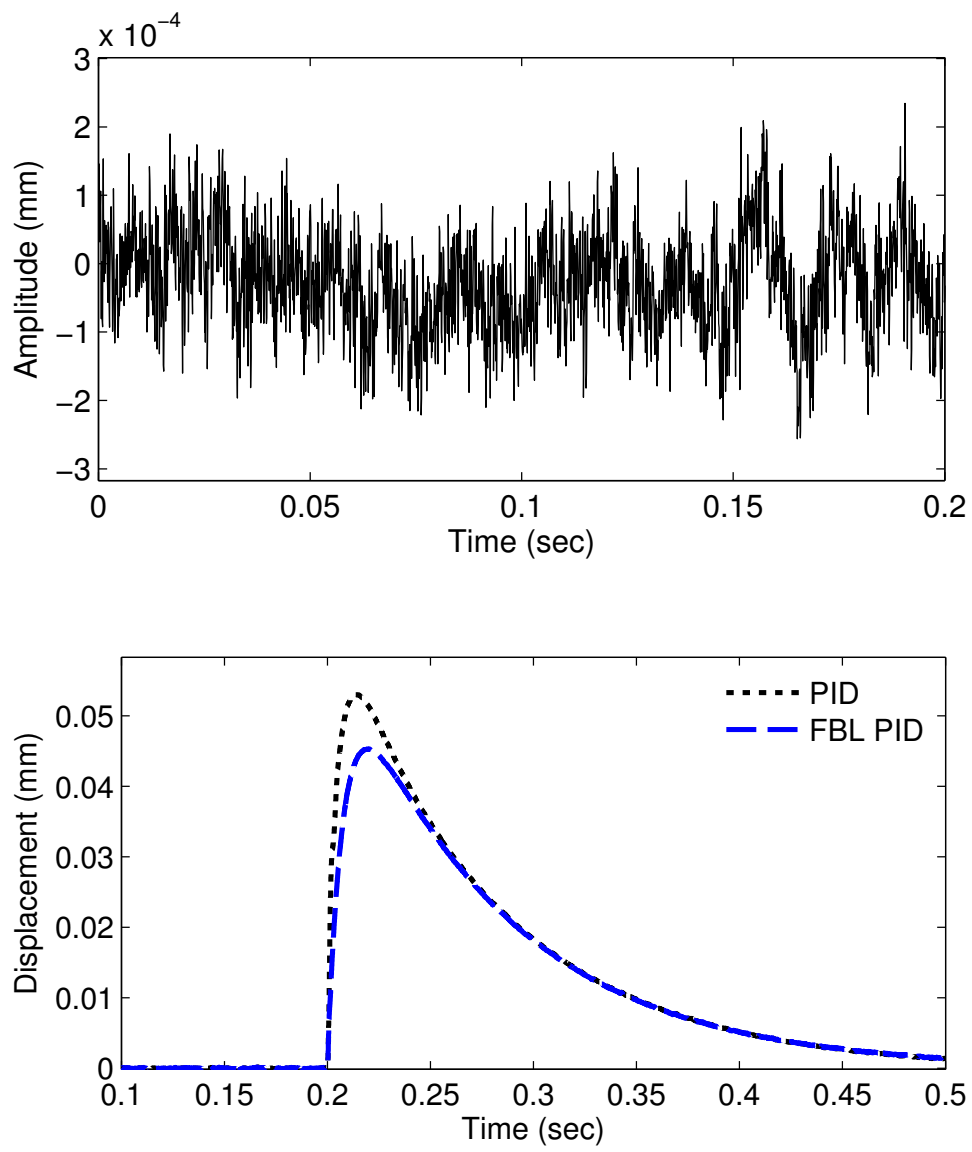

Figure 4.23: Recorded noise signal and simulated response to $1000 \mathrm{~N}$ disturbance step input with sensor noise added to position output signal. 

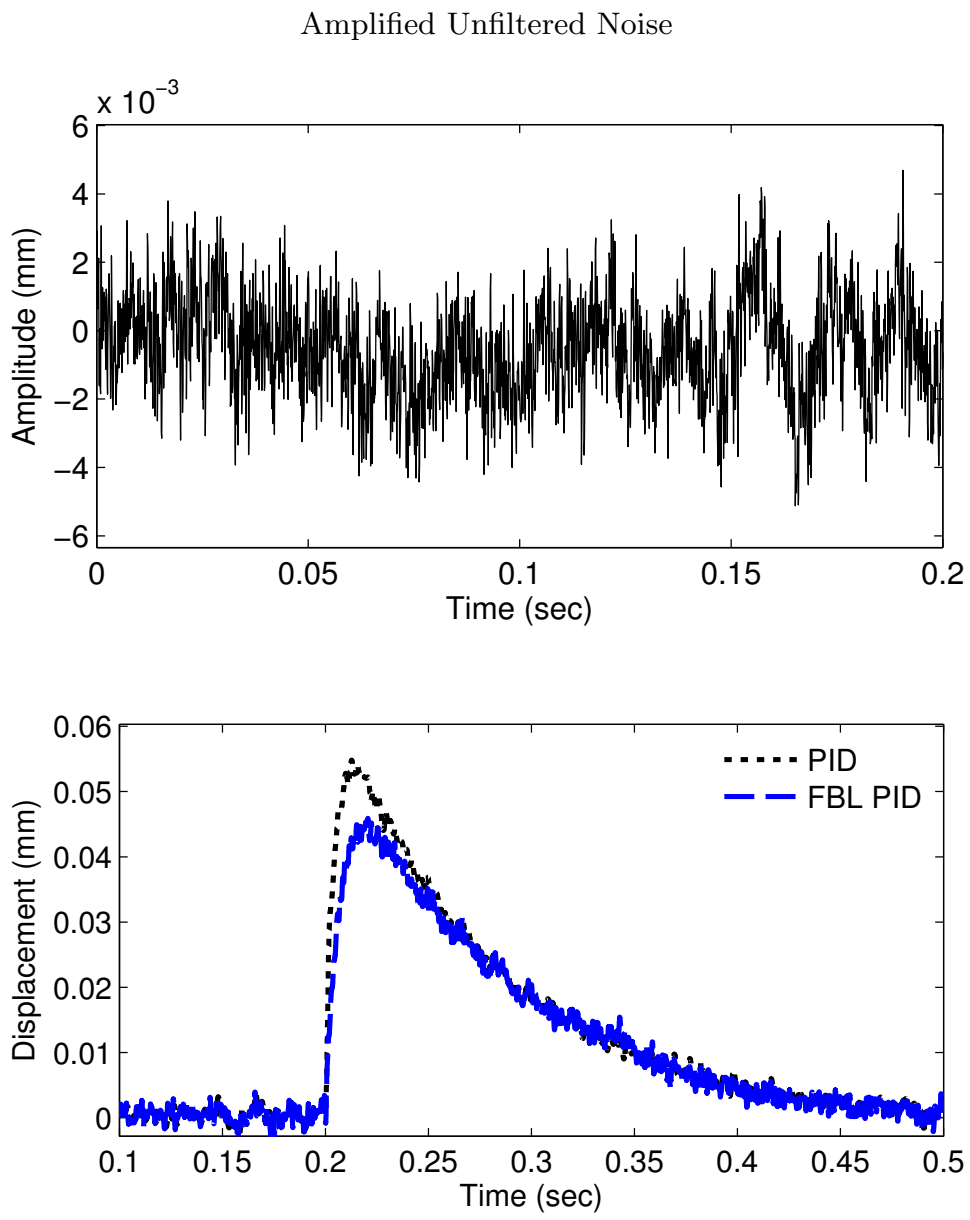

Figure 4.24: Amplified noise and simulated response to $1000 \mathrm{~N}$ disturbance step input with amplified sensor noise added. 


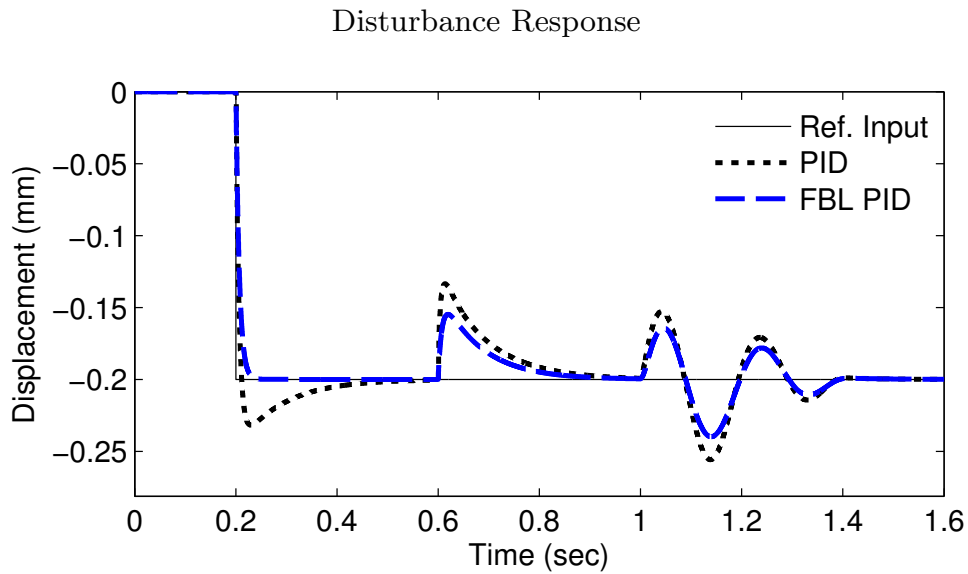

Disturbance Load

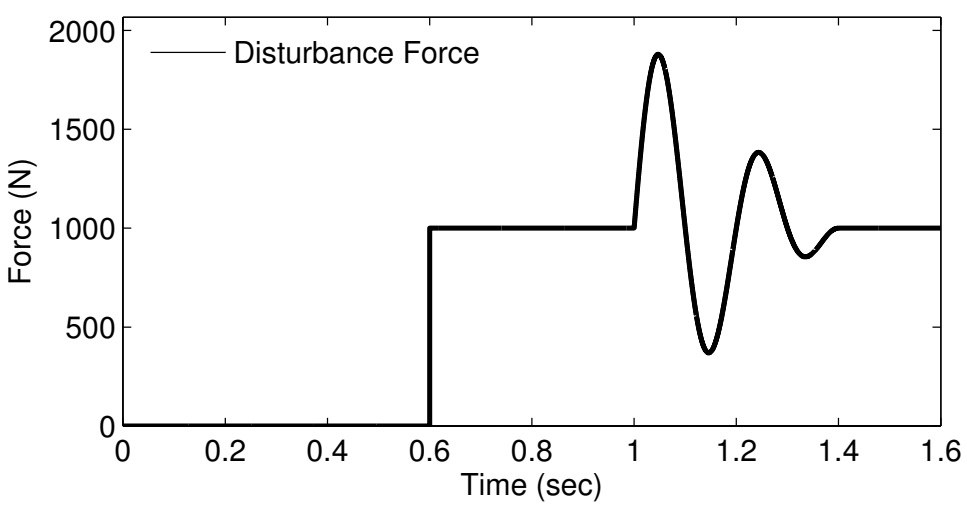

Figure 4.25: Frequency response for disturbance rejection while operating off-center.

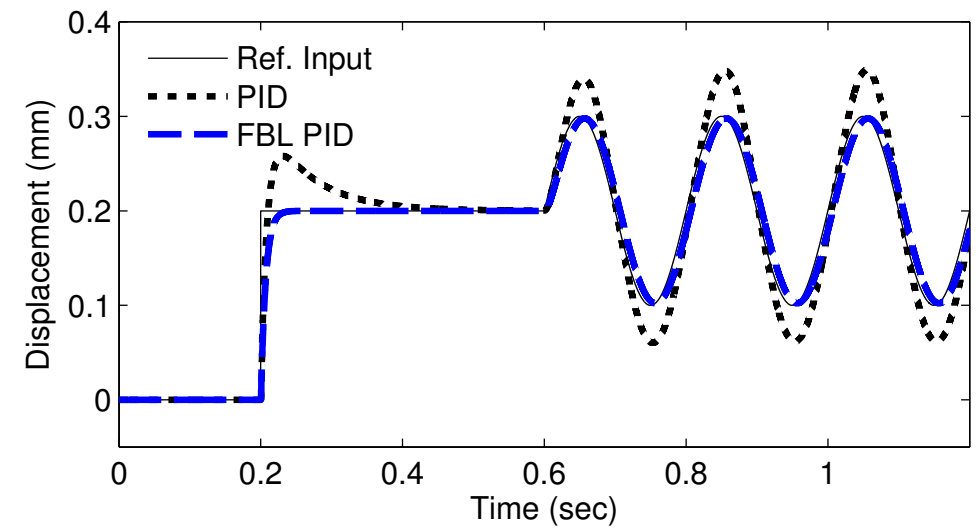

Figure 4.26: Tracking performance of a sinusoidal reference while operating off-center. 

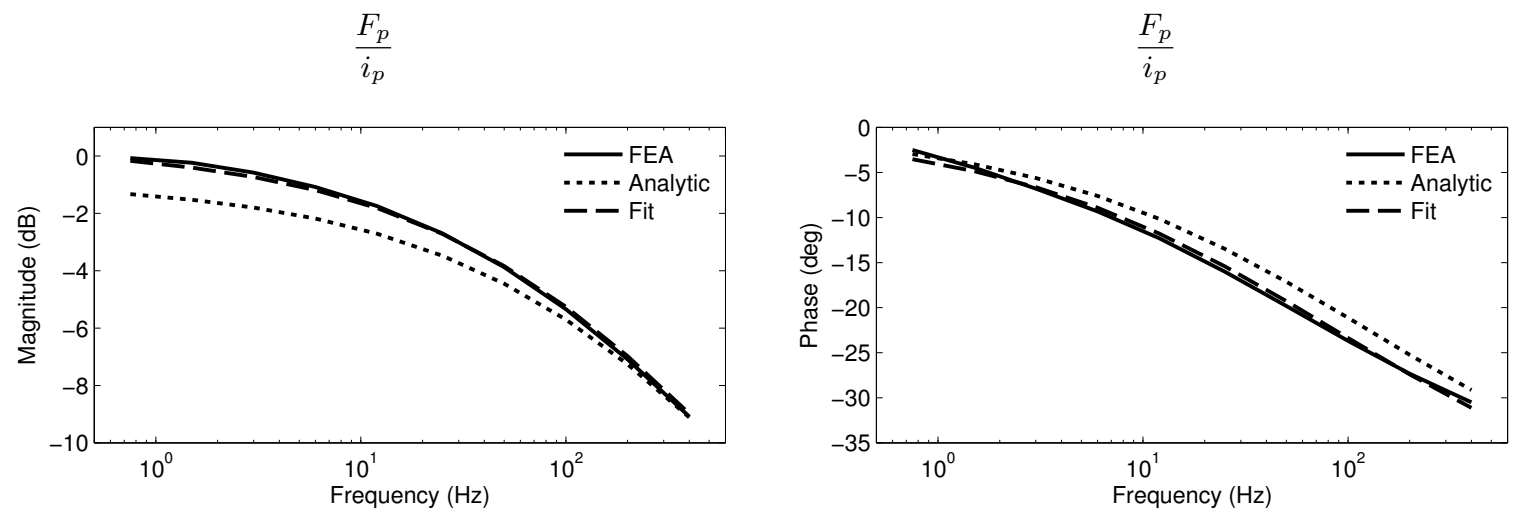

Figure 4.27: Frequency response of FEA model, approximate analytic model and approximate analytic model with $c$ and $R^{0}$ optimized to fit FEA.

Bounds of Disturbance Response

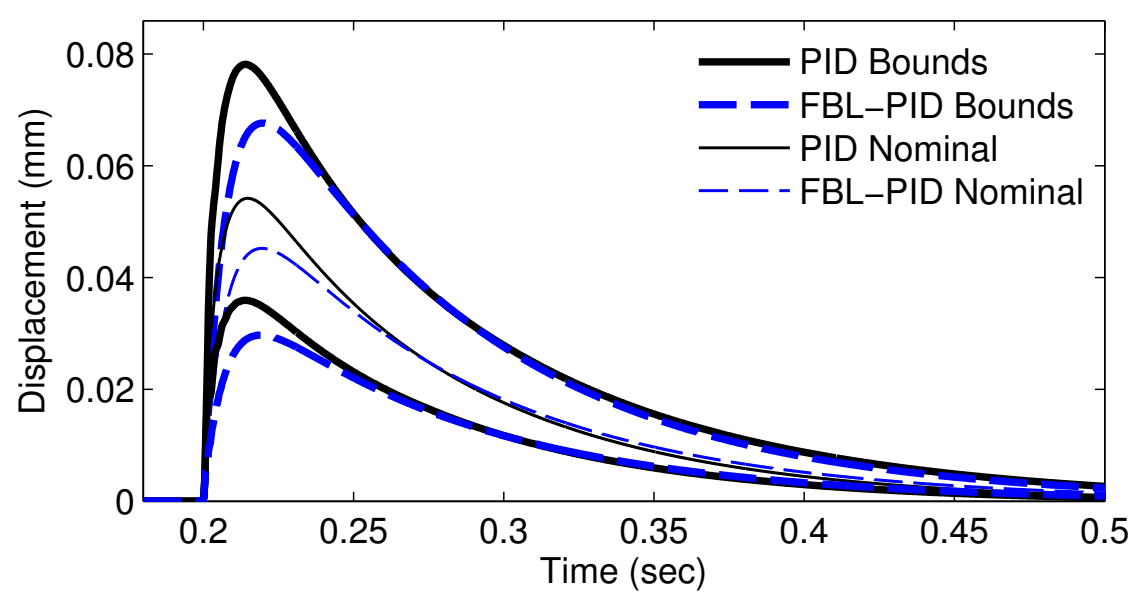

Parameter Distribution
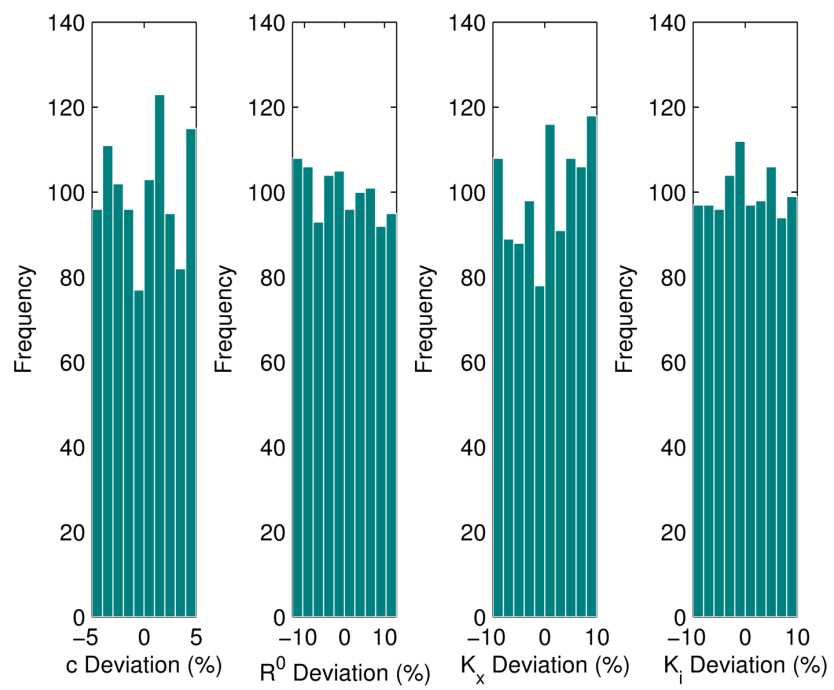

Figure 4.28: Monte Carlo analysis of robustness to model variation with a $1000 \mathrm{~N}$ step disturbance force for 1000 combinations of plant parameters. The disturbance response shows upper and lower bounds for each control type. Nominal disturbance response based on linear analysis is also shown. Plant model parameters were varied according to the distribution shown. 


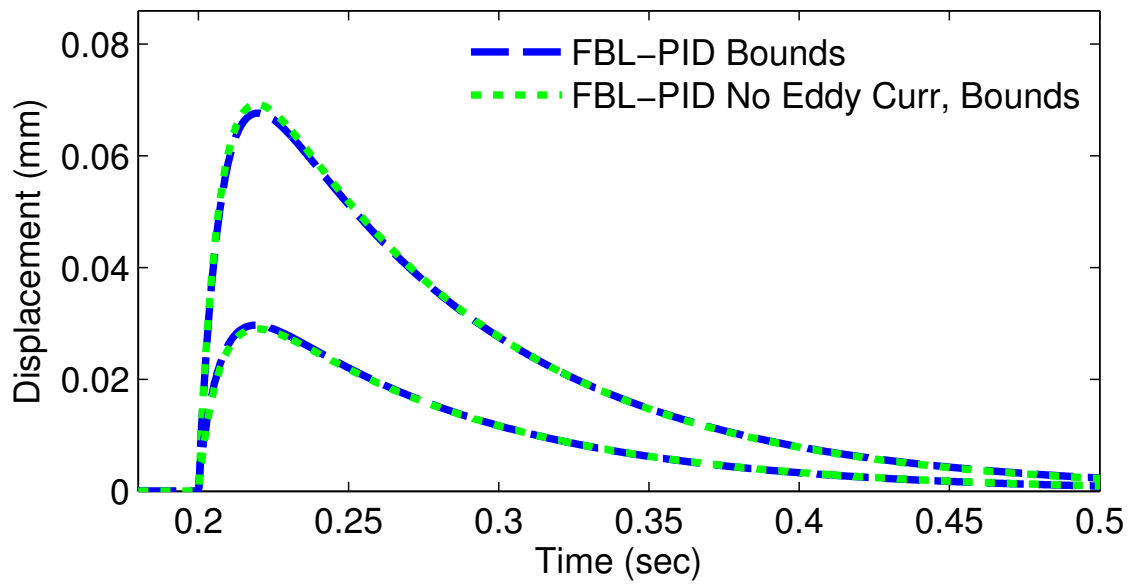

Figure 4.29: Monte Carlo analysis for FBL-PID control of an idealized thrust AMB without eddy current effects and a typical thrust AMB with eddy current effects. The disturbance response shows upper and lower bounds for actuator.
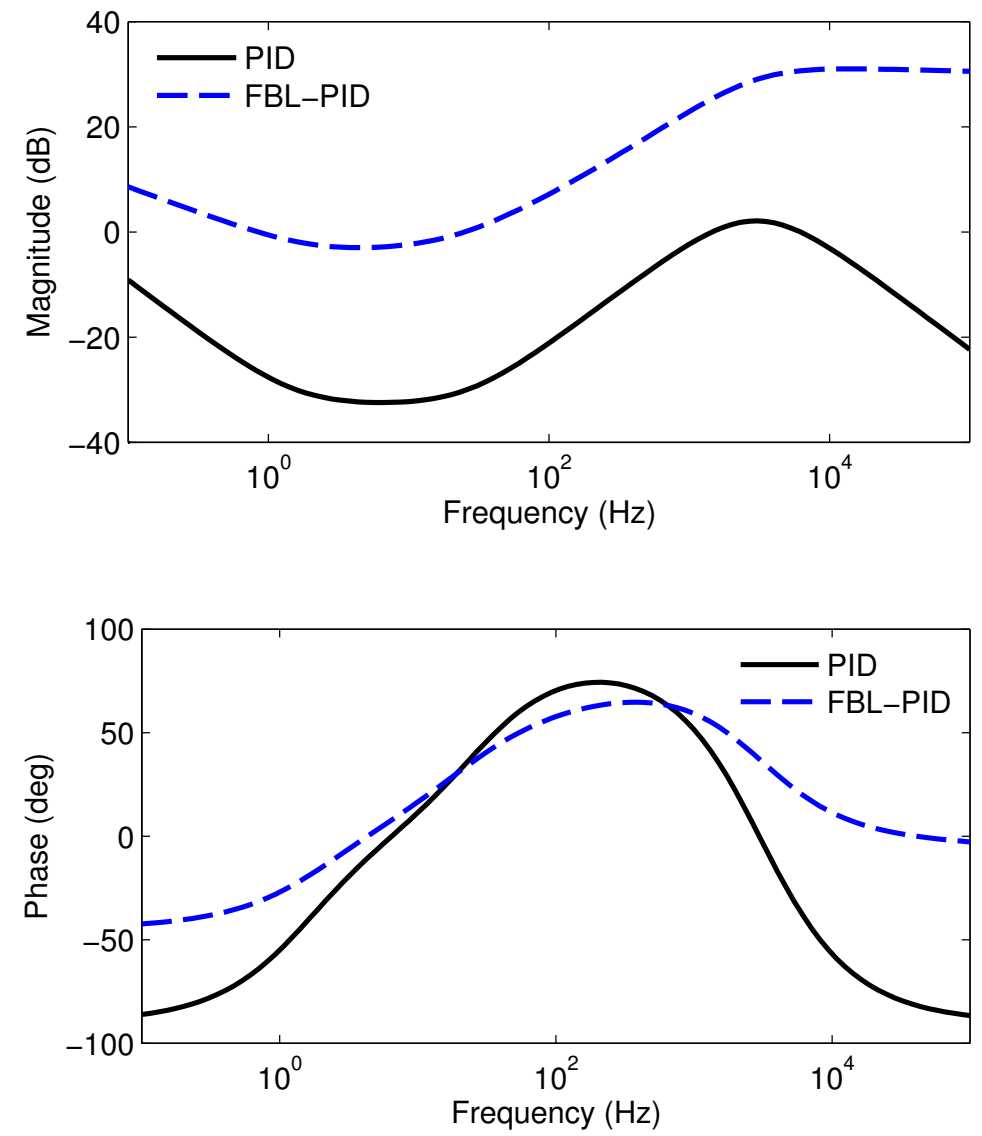

Figure 4.30: Frequency response for PID and FBL-PID control $\left(\frac{F}{m m}\right)$ 
Static

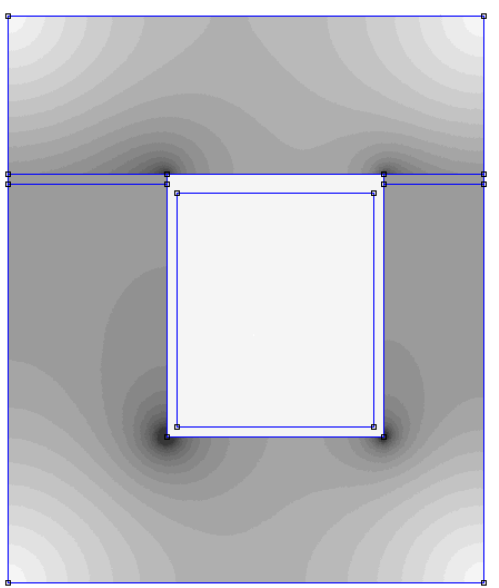

$20 \mathrm{~Hz}$ Perturbation

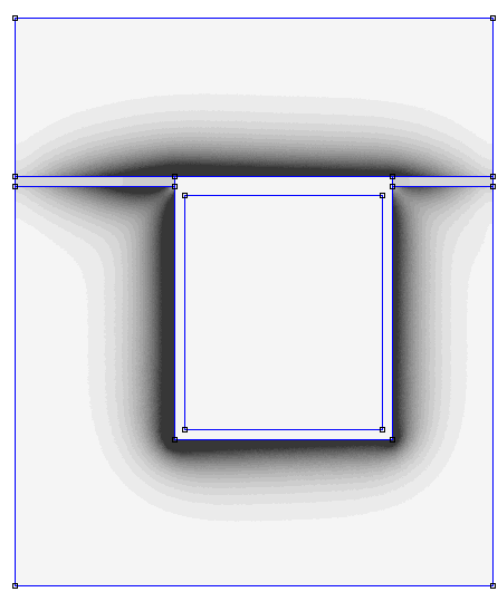

$1.900 \mathrm{e}+000:>2.000 \mathrm{e}+000$

$1.800 \mathrm{e}+000: 1.900 \mathrm{e}+000$

$1.700 \mathrm{e}+000: 1.800 \mathrm{e}+000$

$1.600 \mathrm{e}+000: 1.700 \mathrm{e}+000$

$1.500 \mathrm{e}+000: 1.600 \mathrm{e}+000$

$1.400 \mathrm{e}+000: 1.500 \mathrm{e}+000$

$1.300 \mathrm{e}+000: 1.400 \mathrm{e}+000$ $1.200 \mathrm{e}+000: 1.300 \mathrm{e}+000$

$1.100 \mathrm{e}+000: 1.200 \mathrm{e}+000$

$1.000 \mathrm{e}+000: 1.100 \mathrm{e}+000$

$9.000 \mathrm{e}-001: 1.000 \mathrm{e}+000$

$8.000 \mathrm{e}-001: 9.000 \mathrm{e}-001$

$7.000 \mathrm{e}-001: 8.000 \mathrm{e}-001$

$6.000 \mathrm{e}-001: 7.000 \mathrm{e}-001$

$5.000 \mathrm{e}-001: 6.000 \mathrm{e}-001$

$4.000 \mathrm{e}-001: 5.000 \mathrm{e}-001$

$3.000 \mathrm{e}-001: 4.000 \mathrm{e}-001$

$2.000 \mathrm{e}-001: 3.000 \mathrm{e}-001$

$1.000 \mathrm{e}-001: 2.000 \mathrm{e}-001$

$<0.000 \mathrm{e}+000: 1.000 \mathrm{e}-001$ Density Plot: |B|, Tesla

Figure 4.31: Flux densities for $5 \mathrm{~A}$ current, static and $20 \mathrm{~Hz}$ perturbation. 


\section{Chapter 5}

\section{Conclusions and Future Work}

The goal of this work was to improve performance of non-laminated thrust AMBs. In order to meet this goal, two independent strategies were developed: the first strategy was to improve analytic models for non-laminated thrust bearings and the second strategy was to improve control via nonlinear and dynamic compensation. Accurate analytic models act to facilitate improved design speed and performance, and feedback linearization (FBL) improves performance consistency across the operating range as well as general performance with respect to reference tracking and disturbance rejection.

Analytic modeling is especially critical for thrust AMBs with segmented stators where the only alternative is computationally intensive 3-D FEA which can take hundreds of hours for analysis of a particular geometry. In contrast, computation of the analytic model for a wide range of frequencies takes only milliseconds. Modeling results of thrust AMBs confirmed that dynamic performance can be significantly improved by stator segmentation. The analytic model offers a relatively quick method of determining the number of stator cuts required to achieve a desired performance specification. An accurate analytic model also lends itself to use in the design of FBL compensation.

Feedback linearization has been shown previously to compensate for AMB air gap nonlinearities, making performance more consistent over the operating range. In this work, FBL is employed to improve dynamic force capability as well as compensate for air gap nonlinearities. Using a detailed nonlinear plant model, simulation results showed performance improvements in reference tracking and disturbance rejection for FBL. FBL could also be implemented in parallel with stator segmentation to further maximize performance. 


\subsection{Analytic Models}

An analytic model for thrust AMBs was developed by extending the work of Zhu et al. on modeling of solid

cylindrical electromagnetic actuators $[3,2]$. Modification of the boundary conditions for Zhu's axisymmetric model led to the full analytic model for axisymmetric geometries with a center hole, i.e. non-laminated thrust AMBs. An approximate analytic model was also developed using Taylor Series and textitad hoc approximation methods. The full analytic and approximate models were found to agree closely with FEA modeling. FEA models including flux leakage and fringing also agreed closely with the analytic models.

Having developed an accurate analytic model for a nominal thrust AMB geometry, attention was focused on adapting Zhu's C-type model for segmented thrust AMBs. Using magnetic circuit theory, adaptation of the C-type model to segmented thrust bearing geometries was fairly straight forward. Zhu's model is composed of separate terms for the effective reluctance of the flotor, stator and air gaps. The model for segmented thrust AMBs was developed by assembling these terms as parallel or series components to form a suitable magnetic circuit. Verification of the model by FEA was more complicated and extremely time consuming, reinforcing the need for a simple analytic model.

Harmonic FEA in 3-D is extremely time consuming and computationally intensive. For analysis of eddy current effects, a very fine mesh resolution is required near the material surface surrounding the coil due to the skin effect associated with eddy currents. For stators with one or two cuts, the two-fold symmetry requires that half of the actuator be modeled. The result is a FEA model with millions of nodes, which can take up to 7 days to solve for a single input frequency.

FEA results agreed very well with the analytic model of segmented thrust AMBs for most test cases. However, for geometries with an extremely wide or narrow coil groove, the analytic model was not quite as good. If the static relationship between force and current is known for this category of geometry, then the pole parameters of the model can be adjusted to match the static case with the same parameters used in the analytic model to generate a frequency response. These types of geometries represent extreme cases used for testing the robustness of the model and are unlikely to appear in practical machines.

\subsection{Performance of Segmented Thrust AMBs}

Another benefit arising from this work is a formal analysis of the effect of stator segmentation on the performance of non-laminated AMBs. Thrust AMBs tend to be non-laminated resulting in poor dynamic performance. For a number of AMB applications, non-laminated thrust AMBs have been found to be a performance limiting component. For machines such as compressors and milling spindles, AMBs are often 
subjected to dynamic forces beyond their bandwidth. One solution is to divide the stator into segments arranged like the slices of a pie. It had been previously hypothesized that this would improve dynamic performance, but no formal detailed analysis was available.

FEA presented in Chapter 3 predicts that stator segmentation improves actuator bandwidth significantly. Along with the analytic model, FEA was used to demonstrate that bandwidth may be improved several-fold with the introduction of six to eight stator segments. The first cut has the greatest impact on dynamic performance and performance continues to increase linearly with additional cuts. For typical geometries, the analytic model presented here will greatly facilitate the design process of segmented AMBs.

\subsection{Feedback Linearization and Control}

Another method for improving the performance of non-laminated AMBs is feedback linearization. FBL is the common practice of inverting nonlinearities in a plant to create an artificially linear system that performs more consistently with a single linear controller. Thrust AMBs are highly nonlinear with respect to position and suffer from poor dynamic performance. FBL has been used previously for AMBs with good results but had not been analyzed in combination with a dynamic inversion in an attempt to extend bandwidth.

In Chapter 4, the potential of nonlinear and dynamic compensation to improve performance was analyzed by simulation. A detailed nonlinear model of non-laminated AMBs was developed for this analysis. Simulation results highlighted several advantages which may be obtained via FBL. Control by FBL and PID in combination demonstrated consistent performance across the entire air gap, by following a series of step references from the center position to the stator. With PID control alone, stability was lost once the rotor reached half the distance to the stator from the center. Disturbance rejection was also predicted to improve with FBL. There is always a control design trade-off to be made between reference tracking and disturbance rejection. However, with FBL both can be improved compared to standard PID control.

FBL has been found in some applications to introduce sensitivity to noise or to reduce system robustness. Using recorded sensor noise, nonlinear simulation results suggest that noise is not a significant problem for FBL in non-laminated AMBs. By amplifying the noise signal, it was shown that FBL combined with PID was slightly more sensitive to noise than PID alone. However, for typical noise levels, FBL can be implemented without adverse effects.

Robustness of control by FBL with PID was compared to control via PID alone using Monte Carlo analysis. Plant parameters were varied randomly over a uniform distribution and the response to a step disturbance force input was simulated for 1000 combinations of physical plant parameters. The results demonstrated that 
discrepancies between the plant and the model had similar effects whether control was by FBL combined with PID or by PID alone, suggesting that FBL does not impair system robustness.

\subsection{Future Work}

Analytic models presented in this work were carefully verified by FEA. For the axisymmetric model, predictions agreed very closely with finite element analysis. However, experimental validation was not undertaken. Ideally, experimental results for a variety of geometries would be compared to the analytic model. The model for segmented geometries also agreed closely with FEA for practical ratios of pole-face to coil-face area. Adapting a C-type analytic model to curved geometries opens up the question of how to define pole width and pole length parameters since these are not strictly defined for segmented cylindrical shapes. FEA covering a wider range of geometric parameters would allow a more complete analysis of the relationship between actuator geometry and the pole parameters used to adapt a segmented cylindrical geometry to a C-type model. Leakage and fringing were also neglected in the FEA for segmented geometries due to computational limitations. Leakage and fringing is likely to be different for segmented and whole geometries due to the skin effect. Therefore, FEA including flux leakage and fringing would be useful to determine the accuracy of the analytic model including more realistic conditions such as leakage and fringing.

Experimental validation was not attempted for the model for axisymmetric geometries with a center hole, or for the model for segmented actuator geometries. FEA is extremely useful for testing the accuracy of the analytic models and is a widely used and trusted method for electromagnetic analysis. With FEA, material properties are known and can be made more complex such that results closely match experimental data but have minimal experimental error. However, experimental validation would still be the most definitive test of the models presented in this work.

Control via FBL was not validated experimentally in this work. Simulation was done using a detailed nonlinear model of a non-laminated thrust AMB. But, not all nonlinearities were included in the model; flux saturation, leakage, fringing, and hysteresis were all neglected. The analysis of FBL performance by simulation was promising, suggesting that further investigation and validation using an experimental test rig would be worthwhile. 
Appendices 


\section{A ANSYS Script}

The following is an example script used in ANSYS Mechanical to model a segmented non-laminated thrust AMB.

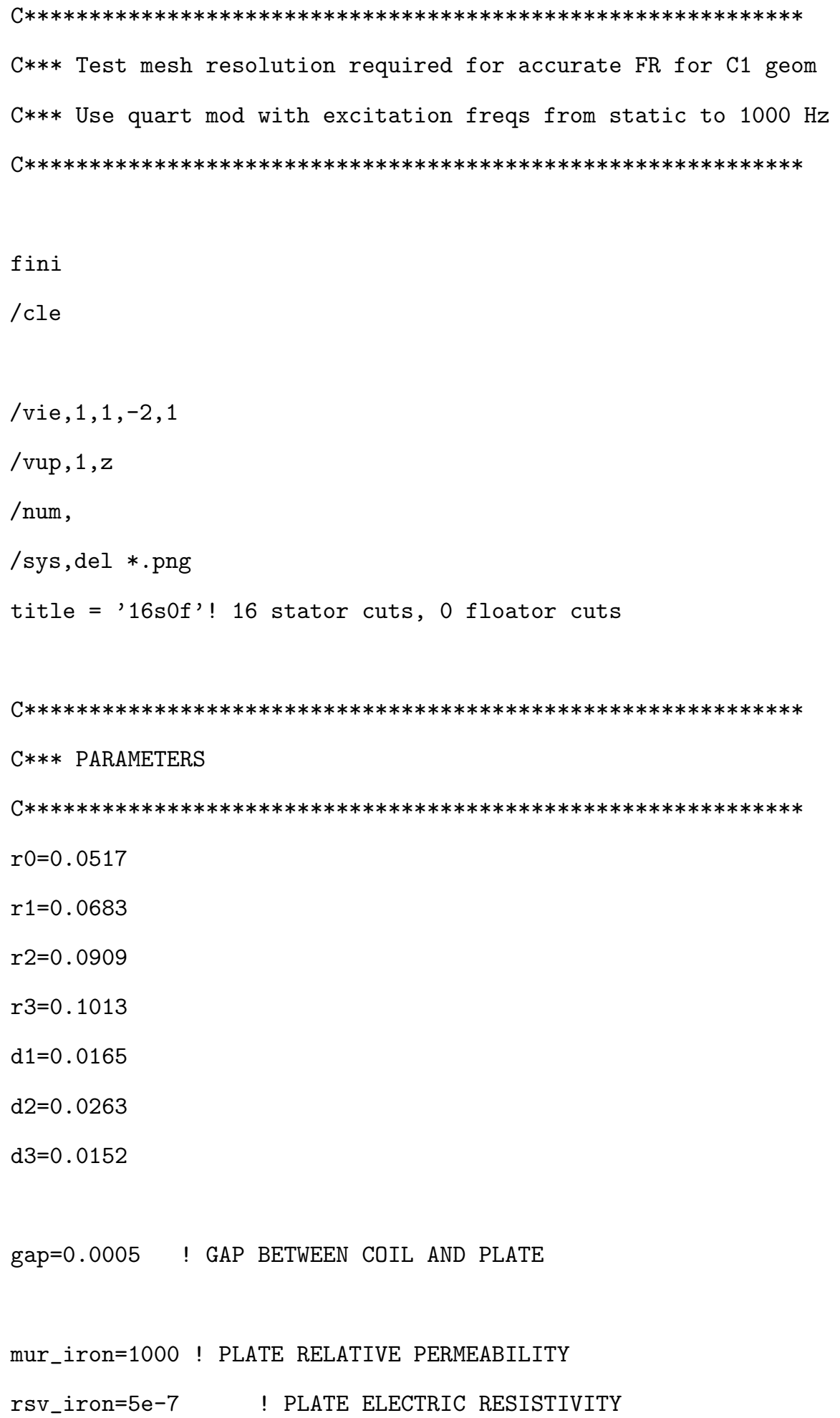


mur_nothing=0.001 ! Relative permeability for coil and surrounding area to prevent fringing

$\mathrm{Ni}=166$ ! COIL SOLENATION

curr_dens $=\mathrm{Ni} /(((r 2-r 1)-0.002) *(\mathrm{~d} 2-0.002)) \quad$ ! CURRENT DENSITY

elem_sz=0.0018

arc=22.5 ! degrees of rotation used in vrotat extrusion command

symm_fact=8 ! symmetry factor is used to multiply B sum in calculation of surface B

! use (division of whole) $/ 2$, ie $2 / 2=1$ for half symmetry model or $4 / 2=2$ for $1 / 4$ symm. model

div_quart=15 ! set number of mesh division used by vrotat command

$\mathrm{C} * * * * * * * * * * * * * * * * * * * * * * * * * * * * * * * * * * * * * * * * * * * * * * * * * * * * * * * * * * * * * * *$

C*** GEOMETRY

$\mathrm{C} * * * * * * * * * * * * * * * * * * * * * * * * * * * * * * * * * * * * * * * * * * * * * * * * * * * * * * * * * * * * *$

/prep7

vsel, none ! BACK IRON

wpcs , $-1,0$

$\mathrm{k}, 0, \mathrm{r} 0,0,0$ ! keypoints $1-10$, outer edge

$\mathrm{k}, 0, \mathrm{r} 0,0, \mathrm{~d} 3$

$\mathrm{k}, 0, \mathrm{r} 0,0, \mathrm{~d} 3+\mathrm{d} 2$

$\mathrm{k}, 0, \mathrm{r} 0,0, \mathrm{~d} 3+\mathrm{d} 2+\mathrm{gap}$

$\mathrm{k}, 0, \mathrm{r} 0,0, \mathrm{~d} 3+\mathrm{d} 2+\mathrm{d} 1+\mathrm{gap}$

$\mathrm{k}, 0, \mathrm{r} 3,0, \mathrm{~d} 3+\mathrm{d} 2+\mathrm{d} 1+\mathrm{gap}$

$\mathrm{k}, 0, \mathrm{r} 3,0, \mathrm{~d} 3+\mathrm{d} 2+\mathrm{gap}$

$\mathrm{k}, 0, \mathrm{r} 3,0, \mathrm{~d} 3+\mathrm{d} 2$

$\mathrm{k}, 0, \mathrm{r} 3,0, \mathrm{~d} 3$

$\mathrm{k}, 0, \mathrm{r} 3,0,0$

k,0,r1,0,d3 ! keypoints $11-22$

$\mathrm{k}, 0, \mathrm{r} 1,0, \mathrm{~d} 3+0.001$

$k, 0, r 1,0, d 3+d 2-0.001$

$\mathrm{k}, 0, \mathrm{r} 1,0, \mathrm{~d} 3+\mathrm{d} 2$ 


$$
\begin{aligned}
& \mathrm{k}, 0, \mathrm{r} 1,0, \mathrm{~d} 3+d 2+\mathrm{gap} \\
& \mathrm{k}, 0, \mathrm{r} 2,0, \mathrm{~d} 3+\mathrm{d} 2+\mathrm{gap} \\
& \mathrm{k}, 0, \mathrm{r} 2,0, \mathrm{~d} 3+\mathrm{d} 2 \\
& \mathrm{k}, 0, \mathrm{r} 2,0, \mathrm{~d} 3+\mathrm{d} 2-0.001 \\
& \mathrm{k}, 0, \mathrm{r} 2,0, \mathrm{~d} 3+0.001 \\
& \mathrm{k}, 0, \mathrm{r} 2,0, \mathrm{~d} 3 \\
& \mathrm{k}, 0, \mathrm{r} 2-0.001,0, \mathrm{~d} 3 \\
& \mathrm{k}, 0, \mathrm{r} 1+0.001,0, \mathrm{~d} 3
\end{aligned}
$$

$\mathrm{k}, 0, \mathrm{r} 1+0.001,0, \mathrm{~d} 3+0.001$ ! keypoints $23-26$, around coil

$\mathrm{k}, 0, \mathrm{r} 1+0.001,0, \mathrm{~d} 3+\mathrm{d} 2-0.001$

$\mathrm{k}, 0, \mathrm{r} 2-0.001,0, \mathrm{~d} 3+\mathrm{d} 2-0.001$

$k, 0, r 2-0.001,0, d 3+0.001$

$k, 0,0,0,-0.01$ ! keypoints for center axis (27 and 28)

$\mathrm{k}, 0,0,0, \mathrm{~d} 3+\mathrm{d} 2+\mathrm{gap}+\mathrm{d} 1+0.01$

$k, 0, r 0+2 *(r 1-r 0) / 3,0,0$ ! keypoints $(29-33)$ for stator section with more coarse mesh

$\mathrm{k}, 0, \mathrm{r} 0+2 *(\mathrm{r} 1-\mathrm{r} 0) / 3,0, \mathrm{~d} 3$

$\mathrm{k}, 0, \mathrm{r} 0+2 *(\mathrm{r} 1-\mathrm{r} 0) / 3,0, \mathrm{~d} 3+\mathrm{d} 2 / 2$

$\mathrm{k}, 0, \mathrm{r} 0,0, \mathrm{~d} 3+\mathrm{d} 2 / 2$

$\mathrm{k}, 0, \mathrm{r} 0+2 *(\mathrm{r} 1-\mathrm{r} 0) / 3,0, \mathrm{~d} 3+\mathrm{d} 2$

$\mathrm{k}, 0, \mathrm{r} 0,0, \mathrm{~d} 3+\mathrm{d} 2+\mathrm{gap}+\mathrm{d} 1 / 2$ ! keypoints $(34-35)$ to divide floater into two regions

$\mathrm{k}, 0, \mathrm{r} 3,0, \mathrm{~d} 3+\mathrm{d} 2+\mathrm{gap}+\mathrm{d} 1 / 2$

1,1,2 ! lines for stator

$1,2,32$

$1,32,3$

$1,3,33$

$1,33,14$

$1,14,13$ 


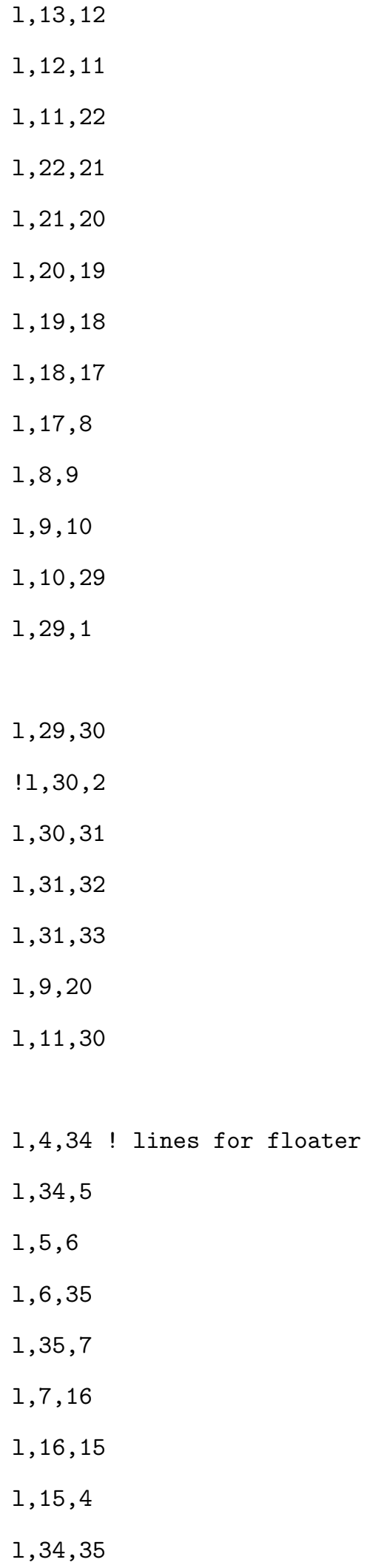


$1,7,8$

$1,14,15$

$1,17,16$

$1,13,24$

$1,25,18$

$1,22,23$

$1,21,26$

1,23,24 ! lines for coil

$1,24,25$

$1,25,26$

$1,26,23$

al,1,2,22,21,20,19 ! Back iron areas

al $, 3,4,23,22$

al $, 21,23,5,6,7,8,25$

al , 20, 25, 9, 10, 11, 24, 17, 18

al $, 12,13,14,15,16,24$

al,26,34,30,31,32,33 ! floater area

al $, 27,28,29,34$

al,35,33,37,5,4 ! air gap areas

al $, 6,37,32,38,14,40,44,39$

al $, 38,31,36,15$

al $, 8,7,39,43,41,9$

al $, 41,46,42,10$

al $, 42,45,40,13,12,11$

al,43,44,45,46 ! coil area

vrotat, $1,2,3,4,5,, 27,28$, arc ! extrude areas to generate back iron vrotat, $6,7,,,,, 27,28$,arc ! extrude areas to generate floater 
vrotat, $8,9,10,11,12,13,27,28$, arc ! extrude areas to generate air gaps vrotat,14,,, ,,27,28,arc ! extrude areas to generate coil

vsel,s,volu,,1,5 ! select back iron and define as component $\mathrm{cm}$, backFe_v, volu vsel,s,volu,,6,7 ! select floater and define as component $\mathrm{cm}$, floater_v, volu

vsel,s,volu,,8 ! select air and define as component vsel, a, volu, , 10 $\mathrm{cm}$, air_v, volu vsel,s,volu,,14 ! select coil and define as component $\mathrm{cm}, \mathrm{coil} \_\mathrm{v}, \mathrm{volu}$ vsel,s,volu,,9 ! select volumes surrounding coil and define as component vsel, a, volu , , 11,13 $\mathrm{cm}$, nothing_v, volu

alls

numm, kp

cmse, all

cmplot

$\mathrm{C} * * * * * * * * * * * * * * * * * * * * * * * * * * * * * * * * * * * * * * * * * * * * * * * * * * * * * * * * * * * * * * *)$ C*** DEFINE ATTRIBUTES

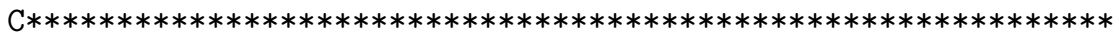

et,1,236,1 ! Back Iron

mp,murx, 1, mur_iron

mp,rsvx, 1,rsv_iron

et,2,236,1 ! Floater

mp,murx , 2,mur_iron 
mp,rsvx , 2,rsv_iron

et, 3,236 ! Air

mp, murx $, 3,1$

et,4,236 ! Coil

mp,murx , 4,mur_nothing

et,5,236 ! Nothing, area immediately surrounding coil

mp, murx, 5, mur_nothing

wpcs,-1,0 ! LOCAL COORDINATE SYSTEMS DEFINE COIL CURRENT DIRECTION

cswp,11,1 ! COIL

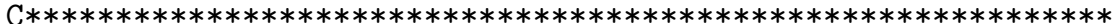

C*** ASSIGN ATTRIBUTES

$\mathrm{C} * * * * * * * * * * * * * * * * * * * * * * * * * * * * * * * * * * * * * * * * * * * * * * * * * * * * * * * * * * * *)$

cmse, s, backFe_v ! Back Iron

vatt $, 1,1,1$

cmse,s,Floater_v ! Floater

vatt $, 2,2,2$

cmse,s,air_v ! Air

vatt $, 3,3,3$

cmse,s,coil_v ! Coil

vatt $, 4,4,4,11$

cmse, s, nothing_v ! Nothing

vatt $, 5,5,5$ 


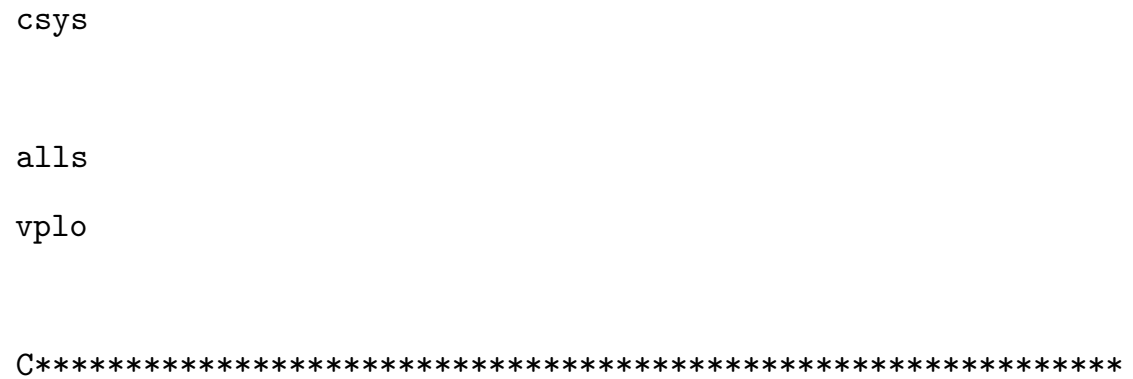




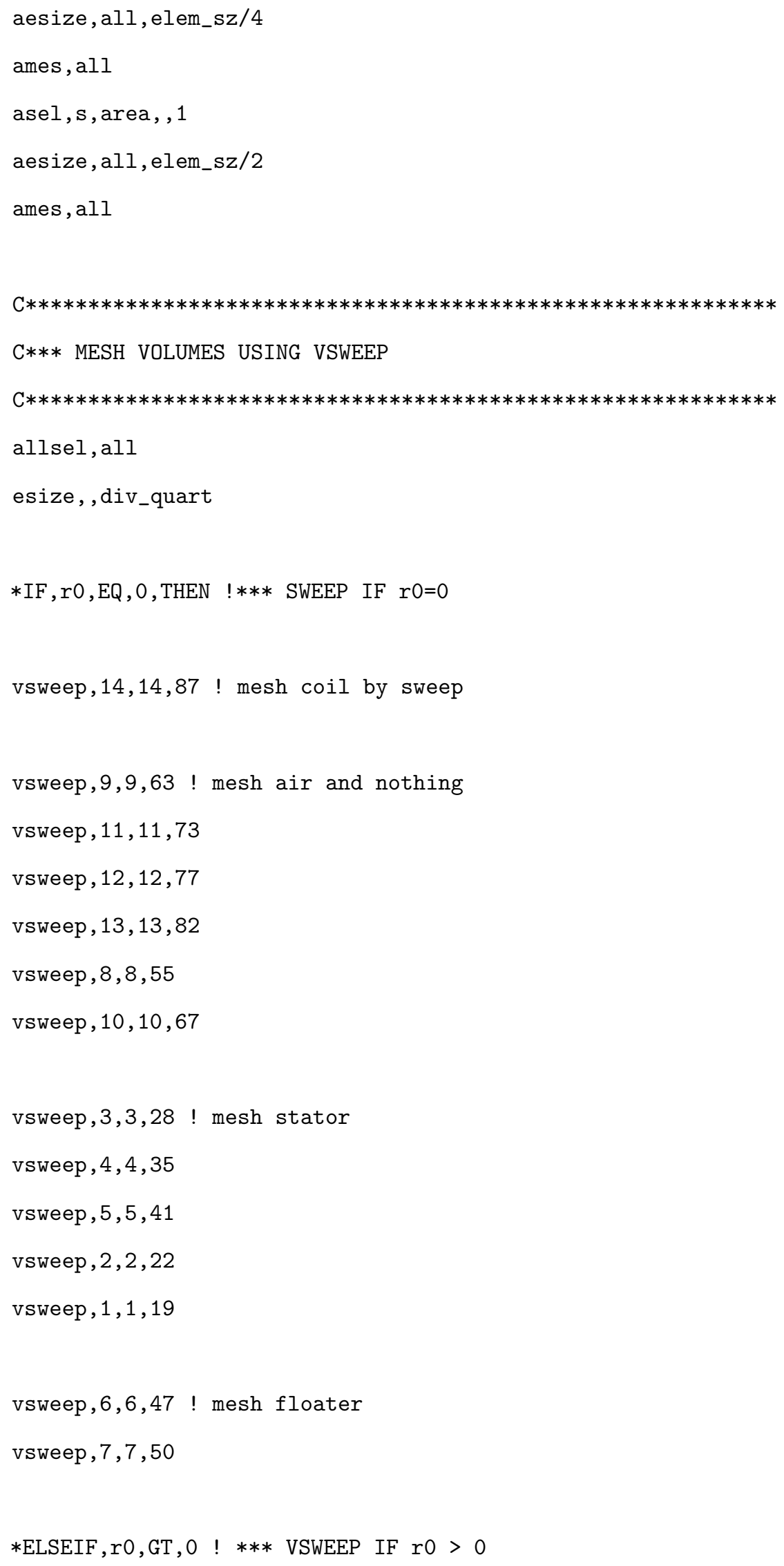


vsweep,14,14,93 ! mesh coil by sweep

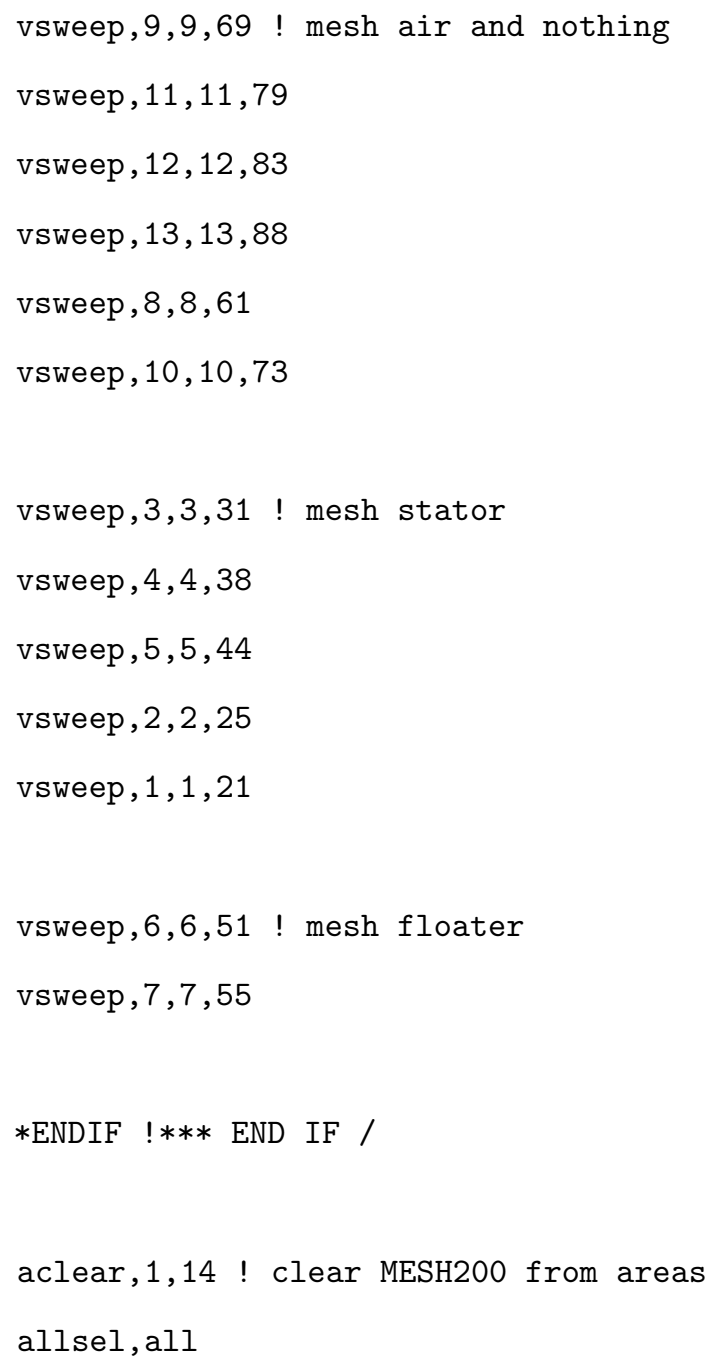


csys ! Set current normal at floater symm. planes, NOT STATOR, ie NO CUTS in floater but stator is cut asel,s,area,,6,7 ! floater BC areas are 6,7,51,55

asel,a,area,,51 ! stator $\mathrm{BC}$ areas are 1-5, and $21,25,31,38,44$

asel, a, area , , 55

! asel,a,area, ,21

! asel,a, area, , 25

! asel,a, area, , 31

! asel, a, area, , 38

! asel, a, area, , 44

da, all, volt

alls ! FLUX PARALLEL EXTERIOR (AZ=0)

asel, s, ext

da, all, az

allsel, all

fini

$\mathrm{C} * * * * * * * * * * * * * * * * * * * * * * * * * * * * * * * * * * * * * * * * * * * * * * * * * * * * * * * * * * * * * * * * *$

C*** Static Solution Goes Here

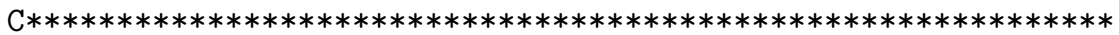

/solu

*cfopen, C: \Users \name \Desktop \ANSYS_DAT.txt

*vwrite,title

(A)

$\mathrm{frq}=0$

*vwrite,frq,par_real

(F10.2, XXXXX,E)

fini 


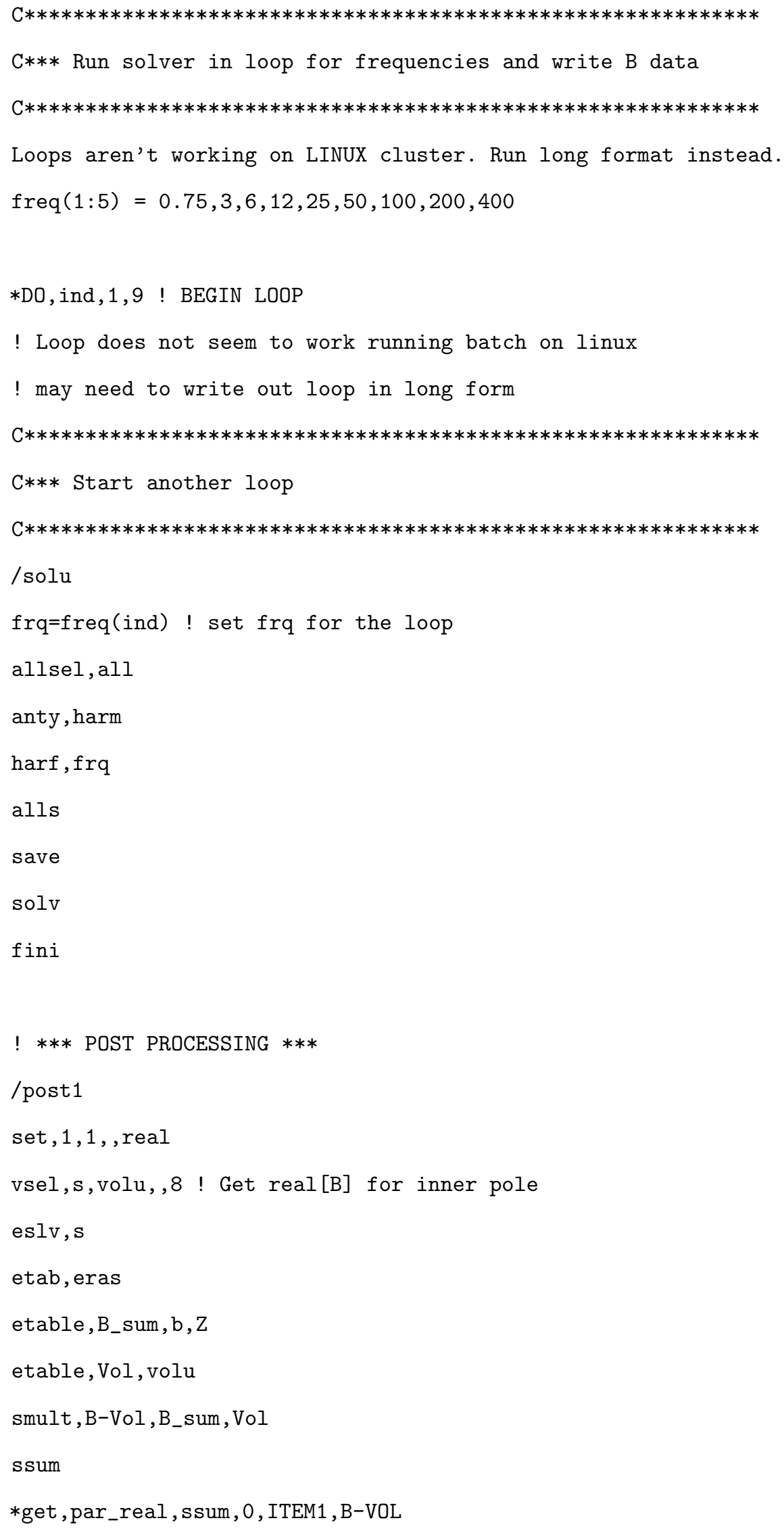


vsel,s,volu,,10 ! Get real[B] for outer pole

eslv,s

etab, eras

etable,B_sum, b, Z

etable, Vol, volu

smult, B-Vol, B_sum, Vol

ssum

*get , tmp , ssum , 0 , ITEM1 , B-VOL

par_real = symm_fact* (par_real - tmp)/gap ! (B_in - B_out) mult. by two because of half symm

! div by two to get flux in single loop of magnetic circuit

! div by gap to get surface flux

vsel,s,volu,,8 ! Get imag[B] for inner pole

eslv, s

set, 1,1, ,imag

etab, eras

etable,B_sum, b, Z

etable, Vol, volu

smult, B-Vol, B_sum, Vol

ssum

*get,par_imag, ssum, 0, ITEM1, B-VOL

vsel,s,volu,,10 ! Get imag[B] for outer pole

eslv, s

etab, eras

etable, B_sum, b, Z

etable,Vol,volu

smult, B-Vol, B_sum, Vol

ssum

*get , tmp , ssum , 0, ITEM1, B-VOL

par_imag $=$ symm_fact* $\left(\right.$ par_imag - tmp)/gap ! (B_in $-B_{-}$out) mult. by two because of half symm

! div by two to get flux in single loop of magnetic circuit

! div by gap to get surface flux 
C*** Write data

$\mathrm{C} * * * * * * * * * * * * * * * * * * * * * * * * * * * * * * * * * * * * * * * * * * * * * * * * * * * * * * * * * * * * * * *$

*vwrite,frq, par_real,par_imag

$(\mathrm{F} 10.2, \mathrm{XXXXX}, \mathrm{E}, \mathrm{E})$

fini

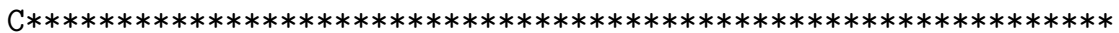

C*** End of freq sol loop

$\mathrm{C} * * * * * * * * * * * * * * * * * * * * * * * * * * * * * * * * * * * * * * * * * * * * * * * * * * * * * * * * * * * * *$

*ENDDO ! END LOOP

$*$ cfclos

fini

/EOF 


\section{B FEMM Lua Script}

Lua scripting was used to run FEMM. The following is an example Lua script.

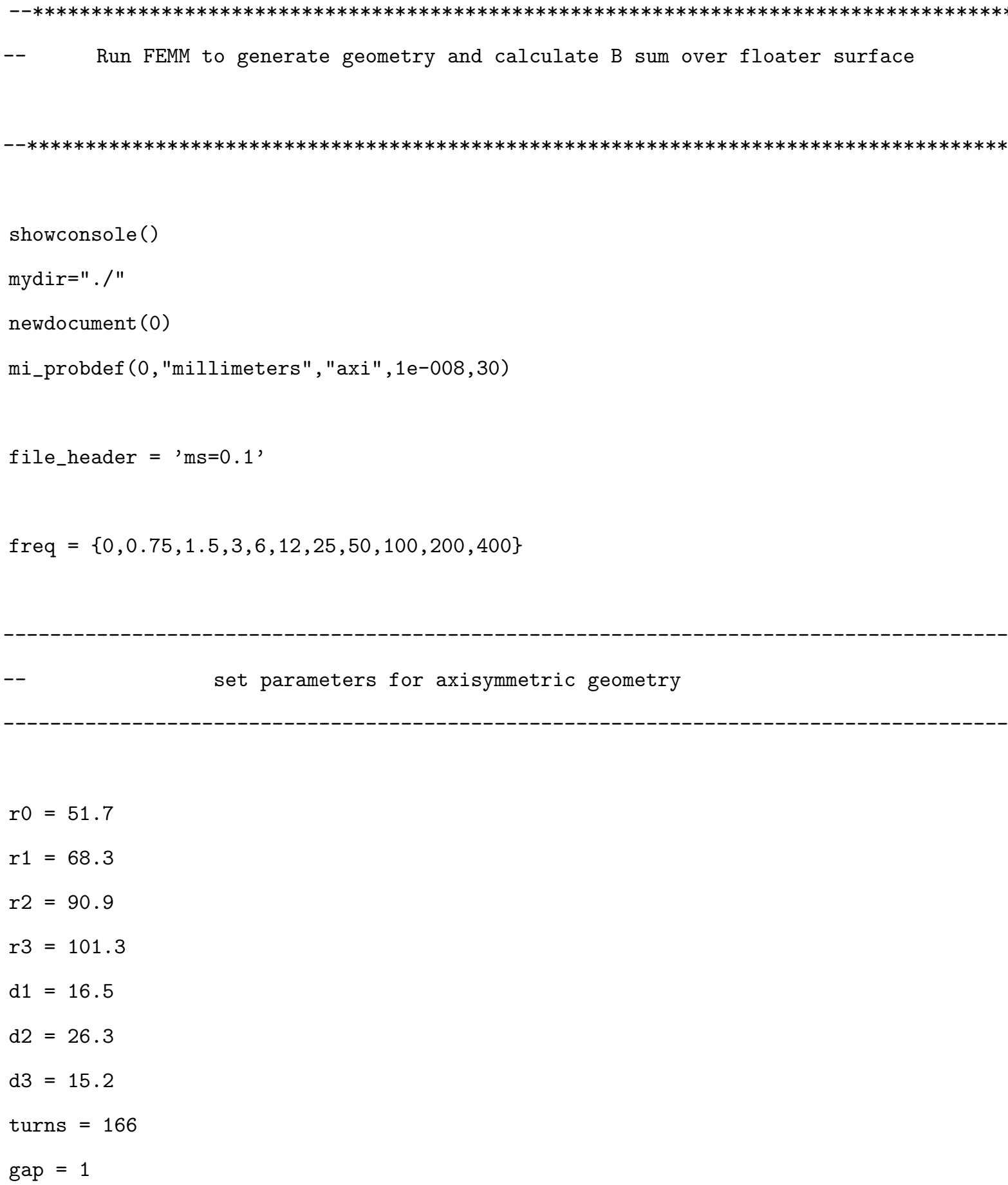


mesh_size $=0.1--0.1$ is good enough up to $400 \mathrm{~Hz}--0.02$ is finest mesh used

set up geometry

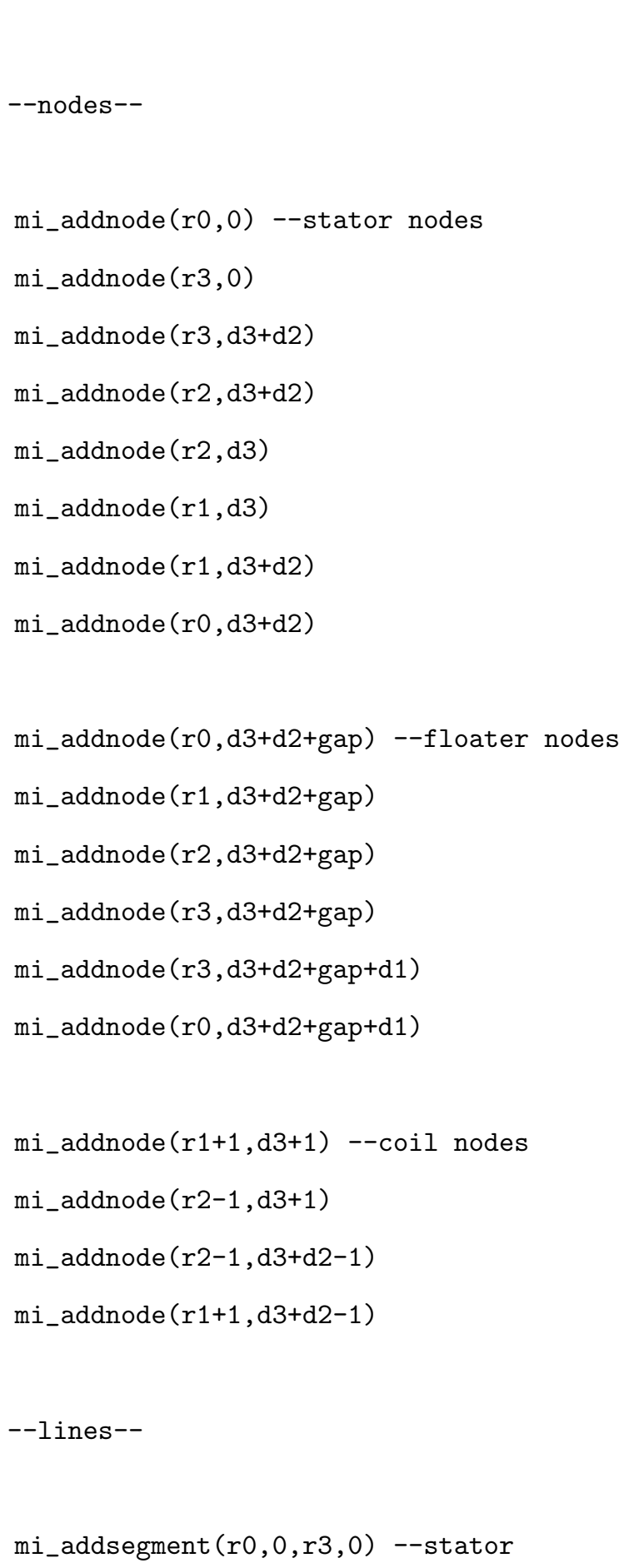




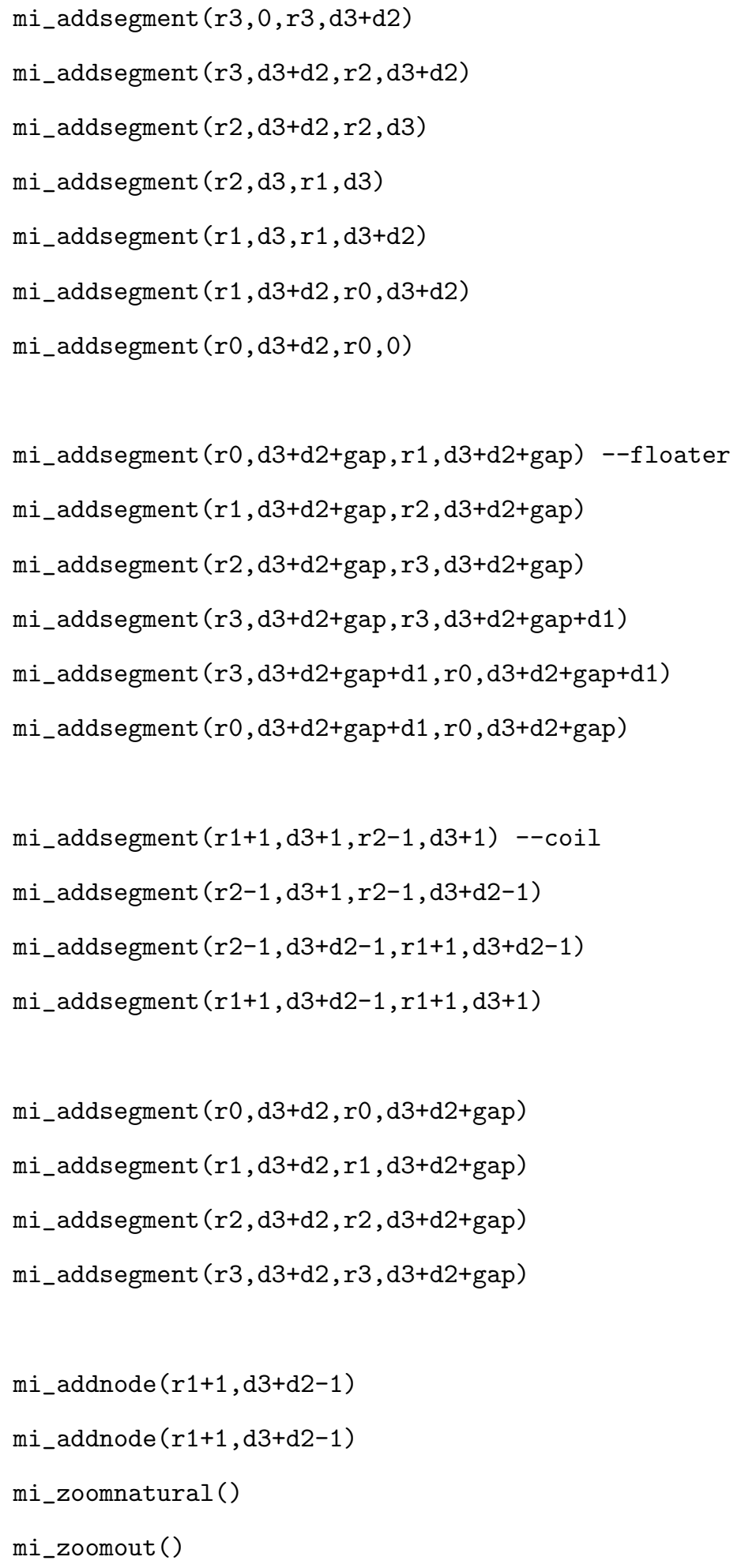




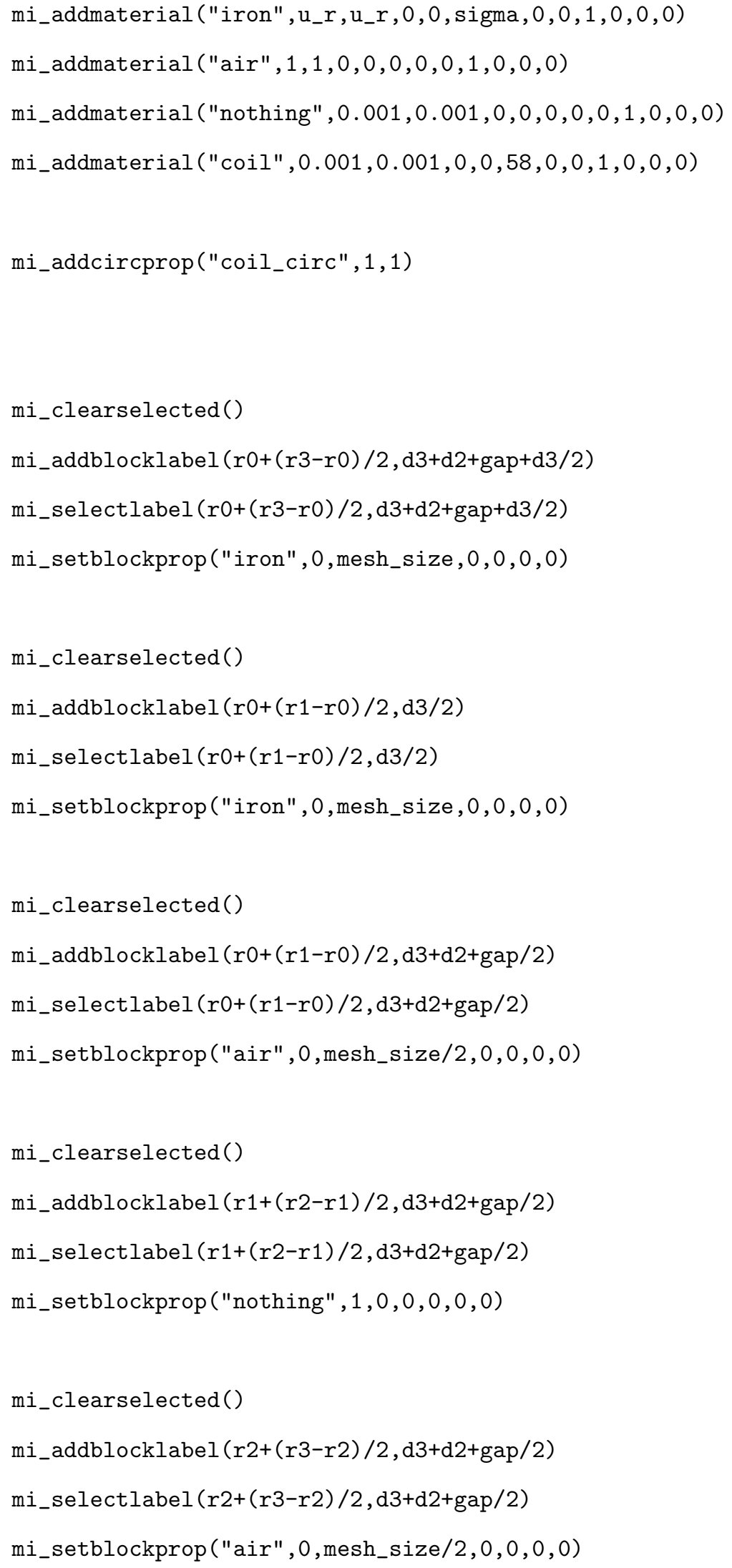




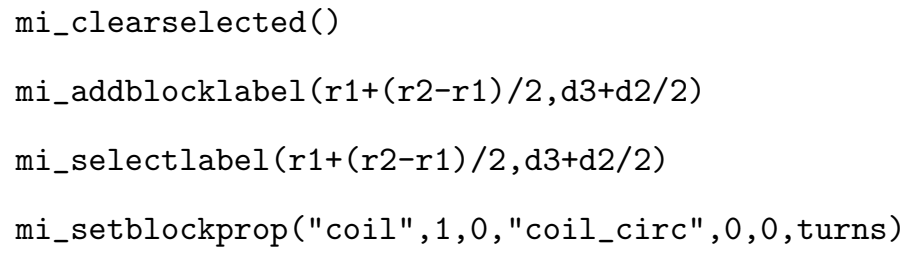




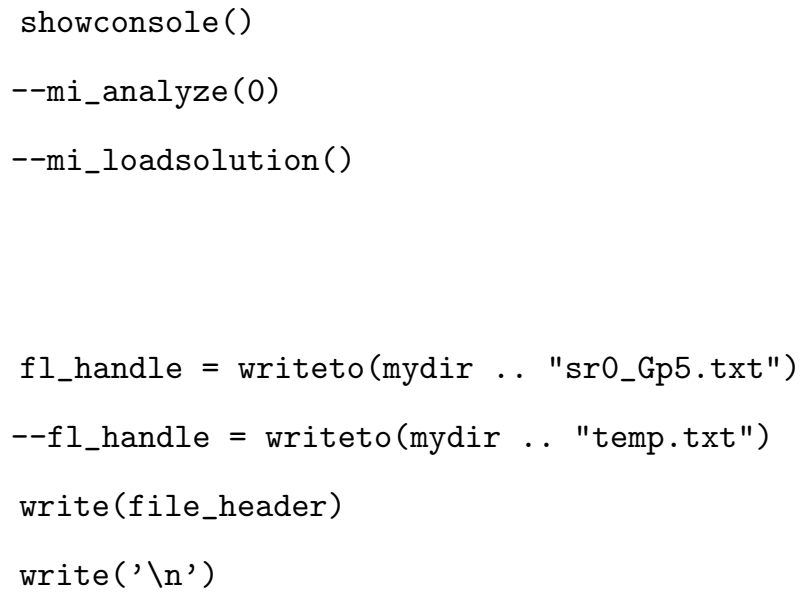


write (Bsurf, '\n')

end

flush (fl_handle)

closefile(fl_handle) 


\section{Bibliography}

[1] Lei Zhu and C.R. Knospe. Modeling of nonlaminated electromagnetic suspension systems. IEEE/ASME Transactions on Mechatronics, 15(1):59-69, February 2010.

[2] Lei Zhu. Non-laminated Magnetic Actuators: Modeling and Performance Limitations. PhD thesis, University of Virginia, May 2005.

[3] Lei Zhu, C.R. Knospe, and E.H. Maslen. Analytic model for a nonlaminated cylindrical magnetic actuator including eddy currents. IEEE Transactions on Magnetics, 41(4):1248-1258, April 2005.

[4] D.W. Lewis P.E. Allaire, and R.R. Humphris. Professor Jesse W. Beams and the first practical magnetic suspension. Proceedings of ISMST1, 1991.

[5] Robert R. Humphris. State-of-the-art of magnetic bearings in rotor systems. ROMAC Report, 1984.

[6] E.H. Maslen. Course notes on magnetic bearings.

[7] Min Chen and Carl R. Knospe. Feedback linearization of active magnetic bearings: Current-mode implementation. IEEE/ASME Transactions on Mechatronics, 2005.

[8] Walter Gordy. Jesse Wakefield Beams. National Academy of Sciences, 1983.

[9] Eric H. Maslen. State-of-the-art and future directions for amb control and applications. ISMB6, 2001.

[10] David J. Griffiths. Introduction to Electrodynamics. Prentice-Hall, 1989.

[11] Matthew O.T. Cole, Eric H. Maslen, Patrick Keogh, Rainer Nordmann, Yohji Okada, Alfons Traxler, Gerhard Schweitzer, Hannes Bleuler, and Rene Larsonneur. Magnetic Bearings. Springer, 2009.

[12] R. L. Stoll. The Analysis of Eddy Currents. Oxford University Press, 1974.

[13] D. K. Anand, R. B. Zmood, and J. A. Kirk. The influence of eddy currents on magnetic actuator performance. Proceedings of the IEEE, 75(2), February 1987.

[14] Myounggyu D. Noh, David C. Meeker, Eric H. Maslen. An augmented circuit model for magnetic bearings including eddy currents, fringing, and leakage. IEEE Transactions on Magnetics, 32(4), July 1996.

[15] Joseph J. Feeley. A simple dynamic model for eddy currents in a magnetic actuator. IEEE Transactions on Magnetics, 32(2):453-458, March 1996.

[16] L. Kucera and M. Ahrens. A model for axial magnetic bearings including eddy currents. Proc. Third Int. Symp. Magnetic Suspension Technology, 2:421-438, December 1995.

[17] Alexsei Tepljakov. Fractional order modeling and control, 2014.

[18] Blas Manuel Vinagre, Dingyü Xue, Vincente Feliu, Concepción Alicia Monje, and Yang Quan Chen. Fractional-order Systems and Controls. Springer, 2010. 
[19] Carl R. Knospe. Feedback linearization: Method and pitfalls. Short Course on Practical Techniques in Control Engineering, April 2006.

[20] Pradeep K. Subrahmanyan, David L. Trumper, and Sean M. Olson. Linearizing control of magnetic suspension systems. IEEE Transactions on Control Systems Technology, 5(4), July 1997.

[21] Jeffrey D. Lindlau and Carl R. Knospe. Feedback linearization of an active magnetic bearing with voltage control. IEEE Transactions on Control Systems Technology, 10(1), 2002.

[22] J. Lindlau. Dynamic Force Biasing of Active Magnetic Bearings Via Feedback Linearization. PhD thesis, University of Virginia, 1999.

[23] David C. Meeker. Finite element method magnetics. Version 4.2 (11Apr2012).

[24] Se Young Yoon. Surge Control of Active Magnetic Bearing Suspended Centrifugal Compressors. PhD thesis, University of Virginia, December 2011.

[25] Zongli Lin, Paule E. Allaire, and Se Young Yoon. Control of Surge in Centrifugal Compressors by Active Magnetic Bearings. Springer, 2013.

[26] Nathan K. Brown. High-Speed Compressor Facility Electromechanical Design. PhD thesis, University of Virginia, January 2005.

[27] Allen R. Tannenbaum John C. Doyle, Bruce A. Francis. Feedback Control Theory. Macmillan, 1992.

[28] Zongli Lin and Parinya Anantachaisilp. An experimental study on pid tuning mmethod for active magnetic bearing systems. Int. J. Advanced Mechatronic Systems, 2013.

[29] L.C. Kammer. Magnetic bearing systems and methods of controlling the same, 2014. 